\title{
Well Installation and Ground-Water Sampling Plan for 1100 Area Environmental Monitoring Wells
}

R. W. Bryce

May 1989

Prepared for the U.S. Department of Energy under Contract DE-AC06-76RLO 1830

Pacific Northwest Laboratory Operated for the U.S. Department of Energy by Battelle Memorial Institute 


\section{DISCLAIMER}

This report was prepared as an account of work sponsored by an agency of the United States Government. Neither the United States Government nor any agency thereof, nor Battelle Memorial Institute, nor any or their employees, makes any warranty, expressed or implied, or assumes any legal liability or responsibility for the accuracy, completeness, or usefulness of any information, apparatus, product, or process disclosed, or represents that its use would not infringe privately owned rights. Reference herein to any specific commercial product, process, or service by trade name, trademark, manufacturer, or otherwise does not necessarily constitute or imply its endorsement, recommendation, or favoring by the United States Government or any agency thereof, or Battelle Memorial Institute. The views and opinions of authors expressed herein do not necessarily state or reflect those of the United States Government or any agency thereof.

\section{PACIFIC NORTHWEST LABORATORY operated by BATTELLE MEMORIAL INSTITUTE for the UNITED STATES DEPARTMENT OF ENERGY under Contract DE-AC06-76RLO 1830}

\begin{tabular}{|c|c|}
\hline \multicolumn{2}{|c|}{ Printed in the United States of America } \\
\hline \multicolumn{2}{|c|}{ Available from } \\
\hline \multicolumn{2}{|c|}{ Narional Technical Informalion Service } \\
\hline \multicolumn{2}{|c|}{ United Staten Department of Commerce } \\
\hline \multicolumn{2}{|c|}{5285 Port Royal Road } \\
\hline \multicolumn{2}{|c|}{ Springtıeld. Virginia 22161} \\
\hline \multicolumn{2}{|c|}{ Nrisprece Codes } \\
\hline \multicolumn{2}{|c|}{ Muerotiche AOr } \\
\hline \multicolumn{2}{|c|}{ Printed Copy } \\
\hline & Price \\
\hline Pingen & Codes \\
\hline $001-025$ & $\mathrm{~A} 02$ \\
\hline $026-050$ & $\mathrm{~A} 03$ \\
\hline $051-075$ & $\mathrm{~A} 04$ \\
\hline $076 \cdot 100$ & $\mathrm{~A} 05$ \\
\hline $107-12.5$ & $A 06$ \\
\hline $126-150$ & $\mathrm{~A} 07$ \\
\hline $151-175$ & $\mathrm{~A} 0 \mathrm{~B}$ \\
\hline $176-200$ & $\mathrm{~A} 09$ \\
\hline $201 \cdot 225$ & A.10 \\
\hline 226.250 & A 11 \\
\hline $251-275$ & A 12 \\
\hline $276-300$ & $A 13$ \\
\hline
\end{tabular}


R. W. Bryce

May 1989

Prepared for the U.S. Department of Energy under Contract DE-AC06-76RL0 1830

Pacific Northwest Laboratory Richland, Washington 99352 


\section{SUMMARY}

This report outlines a plan for the installation and sampling of five we1ls between known inactive waste sites in the 1100 Area of the Hanford Site and Richland City water supply wells. No contamination has been detected in water pumped from the water supply wells to date. The five wells are being installed to provide for early detection of contaminants and to provide data that may be used to make decisions concerning the management of the North Richland Well Field.

This plan describes the existing waste disposal facilities and water supply wells, hydrogeology of the area, well completion specifics, and the data to be gathered from the five new wells. 
. .

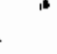




\section{ACKNOWLEDGMENTS}

The author wishes to thank D. A. Lamar, P. E. Long, and R. G. McCain for assembling available information on waste disposal sites near the 1100 Area; G. V. Last for reviewing this report; M. G. Hefty for technical editing; and the sigma $v$ tean for text processing. 
. 


\section{CONTENTS}

SUMMARY. .......................... $\mathbf{i}$ i

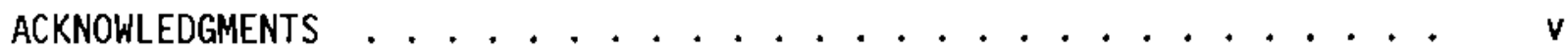

1.0 INTRODUCTION. . . . . . . . . . . . . . . . 1.1

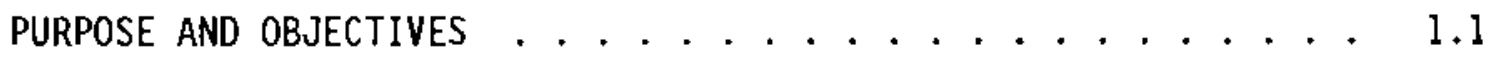

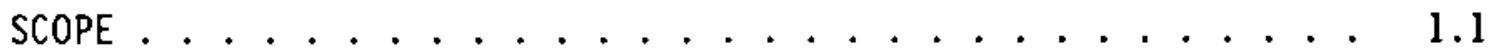

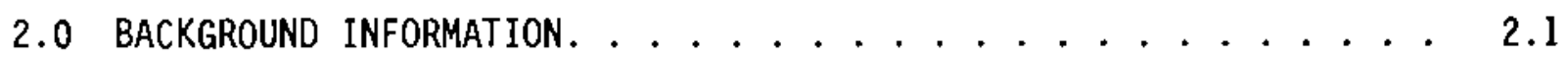

FACILITY DESCRIPTION. . . . . . . . . . . . . . . 2.1

Battery Acid Pit . . . . . . . . . . 2. 2.1

Paint and Solvent Site................ 2.2

Antifreeze and Degreaser Site ......... 2.2

Antifreeze Tank . . . . . . . . . . 2.2

Radioactive Contamination Spill ........ 2.3

Horn Rapids Disposal ............ . . 2.3

Other Waste Sites ............... 2.4

RICHLAND WATER SUPPLY WELLS ........................ 2.5

SUMMARY OF EXISTING DATA . . . . . . . . . . . . . . 2.6

GEOLOGY . . . . . . . . . . . . . . . 2.7

Regional Geology . . . . . . . . . . . 2.8

Local Geology .................. . . 2.8

HYDROLOGY .............................. 2.11

Hanford Site Hydrology . . . . . . . . . . . 2.11

Hydrogeology in the 1100 Area .......... 2.12

3.0 PLAN FOR INSTALLATION AND SAMPLING OF WELLS $\ldots \ldots . \ldots . \ldots$

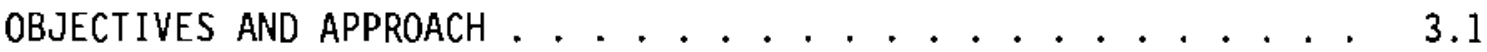




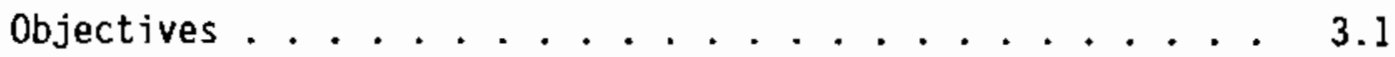

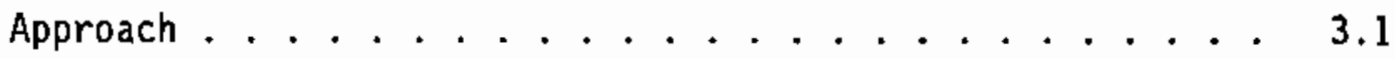

PLACEMENT OF THE FIVE MONITORING WELLS . . . . . . . . 3.2

Drilling and Well Installation .......... 3.6 HYDROGEOLOGIC CHARACTERIZATION $\ldots \ldots \ldots \ldots . \ldots . \ldots$

Geologic Characterization . . . . . . . 3.8

Sampling and Analysis .............. 3.10

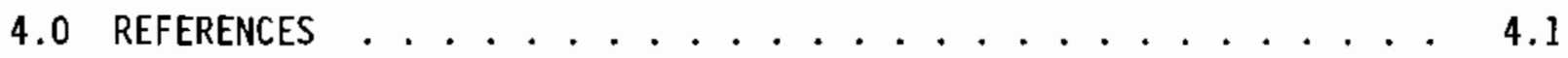

APPENDIX A - FIGURES .................. A.1

APPENDIX B - 1100 AREA WATER QUALITY AND WASTE SITE DATA . . . . . B.1

APPENDIX C - FUNCTIONAL DESIGN AND CONSTRUCT1ON STANDARDS

FOR GROUND-WATER MONITORING WELLS . . . . . . . . . . c. c.

APPENDIX D - DATA COLLECTION AND DOCUMENTATION PROCEDURES FOR

MONITORING WELL INSTALLATION. . . . . . . . . . . . D. 1

APPENDIX E - SAMPLING AND ANALYSIS PROCEDURES . . . . . . . E.1

APPENDIX F - PNL'S QUALITY ASSURANCE PLAN FOR THE HANFORD

GROUND-WATER MONITORING PROJECT AND WESTINGHOUSE

HANFORD COMPANY'S QA APPROVAL OF PNL QA PROGRAM

FOR GROUND-WATER MONITORING WELLS . .......... F.I 


\section{FIGURES}

A.1 Hanford Site Map. .................. A.1

A.2 1100 Area and North Richland Well Field . . . . . . . . A.2

A.3 Structural Setting of the Hanford Site. . . . . . . . . A.3

A.4 Cross Section Showing 1100 Area Stratigraphy. . . . . . . . A.4

A.5 Planned New Well Locations . . . . . . . . . . . A.5

A.6 Drillers Log for Well 119-S41-13C (3000D-1) . . . . . . A.6

A.7 Generalized Diagram of Completion of Shallow Well . . . . . A.7

A.8 Generalized Diagram of Completion of Deep Well . . . . . A.8

IABLES

2.1 Individual Waste Sites in 1100 and 3000 Areas ....... 2.5

2.2 Annual Production of Ground Water from the North Richland Well Field and Volume of Water Pumped into the Recharge Basins from the Columbia River ............ 2.6

3.1 Constituents for which Samples Collected from Wells in the Vicinity of the 1100 Area Will Be Analyzed . . . . . . 3.2

3.2 Recommended Well Depths and Screened Intervals . . . . . 3.6 
" 


\subsection{INTRODUCTION}

\section{PURPOSE AND OBJECTIVES}

The Hanford Site, operated for the U.S. Department of Energy (DOE) by Westinghouse Hanford Company, consists of several functional "areas." The 1100 Area, one of the areas near the city of Richland, has been used for maintenance activities. Waste disposal sites in the 1100 Area received unknown quantities of liquid and solid waste between 1950 and 1985 . While the exact types and quantities of waste are not known, they include spent battery acid, antifreeze, used motor oils, solvents, degreasers, paints, and paint thinners. These disposal sites are close to wells that supply water to Richland, Washington. Although no contaminants have been observed in water samples taken from the water supply wells, five monitoring wells will be installed between the waste disposal sites and the wells supplying water to Richland to detect contaminants in the ground water, should they exist, before they reach the water supply wells. The purpose of this document is to present the plans for the installation and initial sampling of those five monitoring wells.

\section{SCOPE}

The ground-water monitoring system in the vicinity of the 1100 Area and the North Richland Well Field will provide ground-water quality and potentiometric data in the vicinity of the 1100 Area waste disposal facilities and the Richland water supply wells. This plan describes the location of the wells to be drilled, how the wells are to be completed, and the data to be collected. All figures cited in this plan can be found in Appendix A. 


\subsection{BACKGROUND INFORMATION}

\section{FACILITY DESCRIPTION}

The 1100 Area occupies approximately $1.2 \mathrm{mi}^{2}\left(3 \mathrm{~km}^{2}\right)$ at the extreme southeast corner of the Hanford Site, along the northwestern edge of the city of Richland (Figure A.1). It includes equipment storage yards, shipping and receiving, and vehicle maintenance facilities for the DOE's Hanford Site. Individual waste sites known to exist in or surrounding the 1100 Area (see Figure A.2) are briefly described below.

\section{Battery Acid Pit}

From approximately 1957 to 1977, battery acid was disposed of into an unlined pit (i.e., dry sump or French drain) that had sand and gravel placed in the bottom. The pit is located a few feet from a large asphalt-paved area, near the southwest corner of the 1171 Building, which is a vehicle service, maintenance, and repair building. The exact size of the pit is not known, al though estimates by motor-pool workers range from 5 to $12 \mathrm{ft}(1.5$ to $3.6 \mathrm{~m})$ in diameter and 5 to $10 \mathrm{ft}(1.5$ to $3 \mathrm{~m})$ deep. Based on a review of equipment fees and battery procurements by Hanford personnel, the quantity of battery acid disposed of in the pit is estimated to be about 15,000 gal (57,000 1iters). This estimate represents a maximum quantity of battery acid and is based on 1987 battery usage and 1976 vehicle fleet size extrapolated over the period 1957 to 1976 . Other liquid materials, such as solvents, may also have been disposed of in the pit.

Depth to the water table is about $50 \mathrm{ft}(15 \mathrm{~m})$ from ground elevation. During the service life of the pit, acid-saturated sand was occasionally scraped off and disposed of at some unknown location. Fresh sand was then added to the pit. No chemical inventory is available; however, sulfuric acid and lead compounds are the principal contaminants expected. Two surface soil samples have been analyzed, and the results are presented in Appendix $B$ (Table B.1). 


\section{Paint and Solvent Site}

This site was utilized for disposal of solvents, paints, and thinner, as well as solid waste, from 1954 to 1985 . The volume of paint thinner and other solvents disposed of in the pit is estimated to have been approximately 100 gal (380 liters) per year. According to personnel interviews, the solid waste is reported to be broken concrete, asphalt, and lumber from construction and maintenance activities on the Hanford Site.

The site is currentiy covered by approximately $5 \mathrm{ft}(1.5 \mathrm{~m})$ of backfill, mixed with a small quantity of waste asphalt from highway construction conducted in the early 1980s. The site measures about $250 \mathrm{ft}$ long and $100 \mathrm{ft}$ wide $(76 \times 30 \mathrm{~m})$, and the total depth is unknown. No visible evidence of paint, solvent, or solid waste debris exists in the vicinity of this site. The quantity of disposed materials and the exact locations of paint and solvent disposal at this site are unknown.

Depth to the water table is about $50 \mathrm{ft}(15 \mathrm{~m})$ from the ground surface. No chemical inventory is available. Two surface soil samples from the pit reveal no evidence of contamination (see Appendix B).

Antifreeze and Degreaser Site

The Antifreeze and Degreaser Site, in service from 1979 to 1985, is a shallow excavation about $250 \mathrm{ft}(76 \mathrm{~m})$ in diameter. It is reported to have been excavated for sand and gravel borrow material, with the bottom of the original pit at roughly the present observed depth. Approximately $30 \mathrm{yd}^{3}$ $\left(23 \mathrm{~m}^{3}\right)$ of used roofing gravel and $1 \mathrm{yd}^{3}\left(1 \mathrm{~m}^{3}\right)$ of concrete rubble lie on the relatively level bottom of the borrow pit. The quantity of disposed antifreeze and degreasers, as well as specific disposal locations within the pit, is unknown. No chemical inventory is available. Two surface soil samples were taken and revealed no evidence of contamination (see Appendix B). Depth to the water table is estimated at $50 \mathrm{ft}(15 \mathrm{~m})$ below land surface.

Antifreeze Tank

A 5000-gal (19,000-1iter) underground steel tank located in the 1171 Building was used for disposal of waste antifreeze from before 1978 
unti] 1986 when it was emptied, cleaned, and subsequently removed because of suspected leaking. No information is available on the amount of antifreeze that may have leaked. During excavation of the tank, three soil samples were collected from soils surrounding the tank. Analysis of the soil samples did not reveal antifreeze (ethylene glycol). Depth to the water table is about $50 \mathrm{ft}(15 \mathrm{~m})$ from the land surface.

Radioactive Contamination Spill

On August 24, 1962, radioactive contamination was discovered on 16-ton (15 metric tons) cask and on the truck trailer carrying the cask when it arrived at the 1100 Area. After the truck unloaded other cargo, it was parked in the parking lot "northwest of the 1171 Building." The Hanford Laboratories Operation (HLO) Radiation Incident Investigation Number 62-0-59 indicates that an area approximately $\mathrm{l} \mathrm{ft}(0.3 \mathrm{~m})$ in diameter on the bed of the trailer was contaminated. As a result of the incident, several locations were checked including the 1166 Building loading dock, the PIE terminal in Pasco, Washington, and a parking lot in Baker, Dregon, where the truck was parked for 8 hours. No contamination was detected. Atomic Energy Commission officials in Idaho surveyed a location in Twin Falls, Idaho, and found some contamination that was removed and buried. It is logical that radiation monitors at Hanford carefully checked the ground beneath the trailer after the trailer was removed to a radiation zone. However, HLO 62-0-59 does not explicitly make such a statement.

Preliminary analysis of the water from one of three containers in the cask showed a very high iodine-131 content with a significant bariumlanthanum-140 component indicating contact with green fue1. A ruptured zirconium oxide-plutonium oxide capsule was discovered in the container holding the contaminated water.

Based on the available information, it seems likely that no contamination of the parking lot occurred.

Horn Rapids Disposal

The Horn Rapids Disposal, in service from 1950 to 1970 , is an inactive disposal site for (primarily) paper, office, and construction waste (e.g., 
paint cans, solvents, oils). It is about 1000 by $800 \mathrm{ft}$ ( 300 by $240 \mathrm{~m}$ ), with reported original depths to about $40 \mathrm{ft}(12 \mathrm{~m})$. It is now a designated curlew nesting area, and access is restricted. Depth to the water table is estimated to be $30 \mathrm{ft}(9 \mathrm{~m})$. No detailed waste inventory is available. The description below is based on a brief visual inspection of the site.

One area of the disposal site, about $45 \times 30 \mathrm{ft}(14 \times 9 \mathrm{~m})$, is marked by signs indicating that asbestos is buried there. Nearby two signs, several yards apart, read "Burial Site." What was buried is unknown. At a third location an oblate, flat area several meters across, surrounded by a low berm and occupied by a dark gray-brown mud-1ike substance, exhibits mud cracks. Judging from the lack of surface drainage in the area and from the mud cracks, this site was used for disposal of an unknown liquid material. At a fourth location in the disposal area there is a pit about 15 to $20 \mathrm{ft}$ (5 to $6 \mathrm{~m})$ deep and about $40 \mathrm{ft}(12 \mathrm{~m})$ in diameter that contains unburied used tires.

Interviews with personnel involved in the operation of the Horn Rapids Disposal revealed the following:

1. As many as 200 drums of carbon tetrachloride may have been buried in one of the trenches at the site.

2. Standing water was frequently noted in the burial trenches and mention was made of "springs" in the bottom of the trenches. This may indicate that the waste was very near or in contact with the ground water.

\section{Qther Waste Sites}

Additional waste sites known to exist in the 1100 Area east of Stevens Drive and in the 3000 Area to the north are listed in Table 2.1. Although geographically distinct from the sites described, these sites may contain similar wastes. Many of these sites are located in the area of concern near the North Richland Well Field.

In addition to DOE waste sites, other waste sites or potential sources of contamination exist in the vicinity of the 1100 Area. These include the nuclear fuels processing facility operated by Advanced Nuclear Fuels, the 
TABLE 2.1. Individual Waste Sites in 1100 and 3000 Areas

1100 Area Bus Shop
1100 Area Hazardous Waste Staging Area
1100 Area Underground Steam Pad Tank $\# 2$
1100 Area Underground Steam Pad Tank $\# 3$
1100 Area Used 0il Tank $\# 4$
1100 Area Used $0 i 1$ Tank $\# 5$
1100 Area Used 0i1 Tank $\# 6$
700 Area Waste Solvent Tank
3000 Area 1208 Building Hazardous Waste
Staging Area
3000 Area 1226 Building Hazardous Waste
Staging Area
3000 Area 1234 Building Storage Yard
3000 Area 1240 Building Hazardous Waste
Staging Area
3000 Area Jones Yard Hazardous Waste Staging
Area
3000 Area Sanitary Sewer
3000 Area Underground Used 0il Tank

Lamb Weston potato processing plant, the Richland landfill, and several small businesses, including at least one gas station and one machine shop/ automobile repair facility.

\section{RICHLAND WATER SUPPLY WELLS}

The locations of the Richland Water Supply wells (i.e., Duke Wells, the North Richland Well Field, and well 3000D-5) are shown in Figure A.2. The Duke Wells consist of two wells that have a combined production capacity of 2 million gallons per day ( 8 million liters per day). They are located approximately $1 \mathrm{mi}$ south of the Battery Acid Pit and the site of the Antifreeze Tank.

The North Richland Well Field is located approximately $0.5 \mathrm{mi}(1 \mathrm{~km})$ east of the disposal sites. This well field and well 3000D-5 have a production capacity of 4 million gallons per day $(15 \mathrm{ml} / \mathrm{d})$. The production capacity is increased to 11 million gallons per day $(42 \mathrm{ml} / \mathrm{d})$ by artificially recharging the ground water through the use of recharge basins. Water is pumped into the basins from the Columbia River. Table 2.2 lists annual recharge to the basins from 1983 through June 1988 . The table also lists the 
TABLE 2.2. Annual Production of Ground Water from the North Richland Well Field and Volume of Water Pumped into the Recharge Basins from the Columbia River

\begin{tabular}{lrr} 
Year & $\begin{array}{c}\text { Production } \\
\left(10^{3} \text { gal) }\right.\end{array}$ & $\begin{array}{r}\text { Recharge } \\
\left(10^{3} \text { gal }\right)\end{array}$ \\
\cline { 2 - 3 } 1983 & 252,880 & $2,764,360$ \\
1984 & $1,268,670$ & $3,116,940$ \\
1985 & 981,970 & $2,887,622$ \\
1986 & 725,650 & $2,535,760$ \\
1987 & 703,440 & $2,311,373$ \\
$1988(a)$ & 385,020 & 609,918
\end{tabular}

(a) January through June only.

amount of water withdrawn annually from the North Richland Well Field. These data indicate that the ratio of recharge to production has ranged from as high as 4 to less than 2. Richland plans to reduce the amount of water recharged to approximately equal the amount withdrawn in October or November of 1988 . This reduction is a cost-saving measure and also reflects an end to the city's obligation to provide overflow from the recharge ponds to the Tri-City University Center for irrigation of agricultural lands.

\section{SUMMARY OF EXISTING DATA}

Data pertaining to possible contamination of soil and/or ground water resulting from waste disposal operations in the 1100 Area are limited. Existing data consist of the following: 1) two analyses by the state of Washington of well-head water from Richland's North Richland Well Field and Duke Wells (Table B.5), 2) two analyses by the Hanford Environmental Health Foundation (HEHF) of well-head water from the North Richland Well Field (Table B.3), 3) eleven preliminary analyses of water samples from wells in the 1100 and 3000 Areas and vicinity taken during 1986 (Table B.4), 4) analyses of water samples from seven wells in the vicinity of the 1100 Area conducted in August 1988 (Table B.2), and 5) eight surface soil samples from the 1100 Area (Table B.1). A complete listing of the analytical data obtained from these studies can be found in Appendix B. 
The four analyses of well water from Richland indicate that trihalomethanes (bromoform, bromodichloromethane, and chloroform) are the only regulated compounds detected in samples from the North Richland Well Field. The concentrations of trihalomethanes detected are considerably less than the maximum contaminant leve]. Trihalomethanes are commonly associated with water that has been chlorinated and are not believed to have come from the 1100 Area.

The eleven analyses of samples from wells in the 1100 and 3000 Areas and vicinity also indicated the presence of regulated compounds in the ground water. The data obtained from the 1986 sampling indicate the presence of methylene chloride, bis(2-ethylhexyl) phthalate, and several metals (barium,

cadmium, and lead). However, because of concerns regarding well construction and the collection and analysis of the samples, the data may be unreliable. Analyses of samples taken during August 1988 show that bromodichloromethane, chloroform, 1,1,1-trichloroethane, and trichloroethene are present in the ground water in the vicinity of the 1100 Area. The concentrations of these compounds were al1 at least 20 times less than the maximum contaminant level.

Eight preliminary surface soil samples were taken in March 1988 from several sites in the 1100 Area and vicinity. Of the samples taken from the Battery Acid Pit, the Paint and Solvent Disposal Site, and the Antifreeze and Degreaser Pit, only those from the 8attery Acid Pit had elevated concentrations of regulated compounds. The samples from this site contained elevated levels of lead and possibly slightly elevated levels of mercury, chromium, and arsenic. One sample also contained a measurable concentration of the polychlorinated biphenyl arochlor 1254.

\section{GEOLOGY}

This section provides background information on the geology of the Hanford Site and the 1100 Area in support of the installation and sampling of the five ground-water monitoring wells. The geology of the Columbia Plateau and particularly the Pasco 8asin has been studied in detail for DOE as a part of the siting studies for a deep geologic repository for nuclear waste. The Consultation Draft of the Site Characterization Plan (DOE 1988) 
summarizes much of the information known about the Hanford Site, especially near the 200-West Area, where the candidate repository was located. Studies have also been done in support of nuclear power plant licensing efforts, including those for the Washington Public Power Supply System (Supply System 1981) and the Skagit/Hanford Project (PSPL 1982). More detailed information is available on structural geology and tectonics (Caggiano and Duncan 1983; Reidel et al. 1984; Tolan 1986), on basalt stratigraphy and chemistry (Reidel et a1. 1982; Swanson et a1. 1979), and on sedimentary units interfingered with and overlying the basalts (Bjornstad et al. 1985; Fecht et al. 1985). Regiona] Geology

The Hanford Site lies within the Columbia Plateau, which is generally characterized by a thick sequence of basalt flows. These flows have been deformed, creating broad structural and topographic basins separated by asymmetric anticlinal ridges. Sediments have accumulated in some of these basins. The Hanford Site lies within one of these, the Pasco Basin (Figure A.3).

Principal geologic units within the Pasco Basin include, in ascending order, the Columbia River Basalt Group (Miocene), the Ringold Formation (Miocene-P1iocene), the Plio-Pleistocene unit (P1iocene/Pleistocene), the early "Palouse" soil (Pleistocene), and the Hanford formation (Pleistocene). A regionally discontinuous veneer of recent alluvium, colluvium, and eolian sediments overlies the principal geologic units.

\section{Loca7 Geology}

Figure A.4 illustrates supra-basalt stratigraphy in the Pasco Basin. Direct evidence as to the depth, thickness, and characteristics of sediments and basalts beneath the 1100 Area is 1 imited. The description presented below is based primarily on a log for a test well north of the Horn Rapids Disposal (Newcomb 1972) and on extrapolation of geologic conditions from the 300 Area.

\section{Columbia River Basalt Group}

Basalts of the Columbia River Basalt Group are present below a depth of approximately $170 \mathrm{ft}(52 \mathrm{~m})$. Comprising numerous basalt flows and 
interbedded sediments, the Columbia River Basalt Group extends more than $12,000 \mathrm{ft}(3600 \mathrm{~m})$ below the Hanford Site (DOE 1986).

\section{Ringold Formation}

The Ringold Formation directly overlies the uppermost basalt flows of the Columbia River Basalt Group. The Ringold Formation is a fluvial sedimentary unit that exhibits lateral facies changes, including main river channel facies, overbank facies, and fanglomerate facies. Because of the facies variations and 1 imited data, the stratigraphic relationship between Ringold units observed in the 3000,300 , and 1100 Areas and those observed in the 200 Areas is not completely known.

Newcomb (1958) divided the Ringold Formation into three members, based on exposures at the type section along the southern end of the White Bluffs. These are a "lower blue clay member," "a middle conglomerate member," and an "upper member" of silt and fine sand. The "lower blue clay member" is wellknown in the Richland area and southeastward to Kennewick and Pasco, where it occupies much of the saturated part of the Ringold. Close examination reveals that this zone is actually more silt than clay and that it contains considerable sand, presumably as thin interbeds. Gravel and sand beds are interbedded within the "lower blue clay member," and in some places may predominate, particularly in the main river channel facies (Newcomb et al. 1972).

A complete section of the Ringold Formation is not present in the vicinity of the 1100 Area. The upper member and at least part of the middle conglomerate member have likely been removed by erosion (Lindberg and Bond 1979). In the 300 Area, the Ringold Formation is approximately $150 \mathrm{ft}$ (46 m) thick (Lindberg and Bond 1979). The lower $40 \mathrm{ft}(12 \mathrm{~m})$, which corresponds to Newcomb's (1958) "lower blue clay member," consists of silt and clay with occasional sand and gravel. Above the silt and clay is a complex association of gravel and sand with rare lenses of silt or clay. This unit corresponds to Newcomb's (1958) "middle conglomerate member." The top of the Ringold Formation corresponds approximately with the present Columbia River 
level. Ringold sediments deposited above this elevation were removed by erosion before deposition of the overlying Pasco Gravels (Lindberg and Bond 1979).

A simplified well $\log$ for a test well approximately $0.5 \mathrm{mi}(1 \mathrm{~km})$ north of the Horn Rapids Disposal is given in Newcomb et al. (1972). Total thickness of the Ringold Formation in this well is $144 \mathrm{ft}(44 \mathrm{~m})$. The lower $23 \mathrm{ft}$ $(7 \mathrm{~m})$ correspond to the "lower biue clay member" discussed above, while the remaining thickness consists primarily of gravel, graveliy sand, sand, and sitty sand, with occasional interbeds of clay and siltstone.

\section{Pasco Gravels}

Overlying the Ringold Formation and extending to near the surface are glaciofluvial deposits known as the Pasco Gravels. These gravels were deposited by Pleistocene floods resulting from catastrophic failure of ice dams in western Montana and northern Idaho. The Pasco Gravels were deposited on an irregular erosional surface along main channelways of the catastrophic floods. Thickness of the gravels varies from 30 to more than $50 \mathrm{ft}$ ( 9 to $15 \mathrm{~m})$.

Touchet beds are rhythmical7y bedded, fine-grained, slack water flood facies deposited away from flood bars and generally coeval with the Pasco Gravels. Because the 1100 Area lies along a main flood channelway, Touchet beds are not expected to constitute a significant part of the stratigraphic section. Plio-Pleistocene fanglomerates or paleosols between the Ringold and the Pasco Gravels have not been identified in well logs from the 1100 Area and vicinity.

The well $\log$ for a second well presented by Newcomb et al. (1972) indicates a total thickness of $47 \mathrm{ft}(14 \mathrm{~m})$ for "glaciofluviatile and fluviatile deposits, "corresponding to the Pasco Gravels. These deposits consist of sandy gravel with boulders, in which the predominant lithology of the particles is generally basalt.

\section{Surficial Eolian Sediments}

Eolian sands and silts cover the area as a veneer of varying thickness. Longitudinal dunes that were locally active extend up to or across the 
1100 Area from southwest to northeast. The dunes are up to $10 \mathrm{ft}(3 \mathrm{~m})$ thick and are largely stabilized by vegetation or have been reworked in grading and excavation for plant facilities.

\section{HYDROLOGY}

This section describes the hydrology for the Hanford Site and the 1100 Area.

Hanford Site Hydrology

The Hanford Site has an arid climate and receives an average of $6.25 \mathrm{in}$. $(15.88 \mathrm{~cm})$ of precipitation per year. Evaporation rates are expected to far exceed this amount (DOE 1988); therefore, most precipitation is transpired or evaporated back to the atmosphere (Graham et a]. 1981). Recharge rates range from near zero to more than $4 \mathrm{in.} / \mathrm{yr}(10 \mathrm{~cm} / \mathrm{yr})$, depending on surface conditions (Gee 1987). The larger values are associated with areas having a coarse gravelly surface and no vegetative cover. The Hanford Site is drained by the Yakima and Columbia rivers (Figure A.3).

Ground water beneath the Hanford Site occurs under both unconfined and confined conditions. The unconfined aquifer is contained primarily within the middle unit of the Ringold Formation and extends into the Hanford formation in areas. The base of the unconfined aquifer is the uppermost basalt flow of the Columbia River Basalt Group or, in some areas, the "lower blue clay member" of the Ringold Formation. The confined aquifers beneath the Hanford Site generaliy consist of sedimentary interbeds and interflow zones that occur between dense basalt flows of the Columbia River Basalt Group.

The source of natural recharge to the unconfined aquifer and some of the confined aquifers is rainfall from areas of high relief west of the Hanford Site and from ephemeral streams in the Cold Creek and Dry Creek valleys. The Yakima River also recharges the unconfined aquifer in the southern portion of the Hanford Site. Discharge from the unconfined aquifer is primarily to the Columbia River. 
Hydrogeology in the 1100 Area

Ground water beneath the 1100 Area occurs in confined aquifers within the basalt sequence and in the unconfined aquifer of the Pasco Gravels and the sands and gravels of the Ringold Formation. Perched or semiperched water conditions may also occur locally. The boundary between the confined and unconfined aquifers is the lowermost silt and "lower blue clay member" of the Ringold Formation when it is present (Lindberg and Bond 1979; Newcomb et al. 1972). The depth to the water table in the vicinity of the 1100 Area is estimated to be $50 \mathrm{ft}(15 \mathrm{~m})$.

The unconfined aquifer in the area exhibits relatively high permeability, particularly in the Pasco Gravels. Aquifer pumping tests and numerical ground-water modeling for the 300 Area (see location in Figure A.1) indicate transmissivities greater than $100,000 \mathrm{ft}^{2} / \mathrm{d}\left(9300 \mathrm{~m}^{2} / \mathrm{d}\right.$ ) (Schalla et al. 1988). The storativity of the unconfined aquifer has been estimated to be 0.1 for hydrologic studies of the 300 Area (Schalla et al. 1988). Aquifer tests conducted in the North Richland Well Field (ICF 1987) indicate a transmissivity of approximately $86,000 \mathrm{ft}^{2} / \mathrm{d}\left(8000 \mathrm{~m}^{2} / \mathrm{d}\right)$, and storativity of 0.11 . No measurements of these parameters, or of the dispersive or retardation characteristics of these aquifers beneath the 1100 Area, are avai1able. The proximity of the 1100 Area to the 300 Area and the geologic similarity between the two areas suggest that hydrologic properties of the 1100 Area may be similar to those of the 300 Area.

Along the north edge of the study area, the water table dips to the east and ranges from approximately 370 to $350 \mathrm{ft}(113$ to $107 \mathrm{~m})$ above mean sea leve]. Ground-water flow in the 1100 Area is thought to generally be west to east, controlled by the elevation difference between the Yakima [approximately $370 \mathrm{ft}(113 \mathrm{~m})$ ] and the Columbia rivers [approximately $340 \mathrm{ft}$ $(104 \mathrm{~m})]$. The Yakima River recharges the unconfined aquifer, which in turn discharges to the Columbia River. A number of factors potentially perturb this relatively simple system: 1) spatial differences in hydraulic conductivity of the unconfined aquifer; 2) variations in the river stage of both the Yakima and Columbia rivers; 3) infiltration to the unconfined aquifer from irrigation of farms and lawns, or discharges from industrial operations 
such as the Lamb Weston potato processing plant or Advanced Nuclear Fuels; 4) upward leakage (discharge) from the confined aquifer to the lower part of the unconfined aquifer; 5) operation of the North Richland Well Field; and 6) operation of other domestic and/or commercial wells. Of these factors, the operation of the North Richland Well Field is likely the most significant and is discussed below. Note also that little or no recharge is expected from precipitation because of the low annual precipitation and the relatively high evaporation rate.

Halfway between the 1100 Area and the Columbia River is Richland's North Richland Well Field. Figure A.2 shows the location of this well field. The wells are completed in the unconfined aquifer and are recharged artificially by infiltration ponds. Columbia River water is used to supply the ponds, and the well field is used for the city water supply during peak demand periods.

Intermittent operation of the we1l field and recharge ponds may cause significant fluctuation of the water table and affect the rate and direction of ground-water flow beneath the 1100 Area.

\section{Surface Hydrology}

The major surface water features at the Hanford Site are the Columbia River, which is located approximately $1 \mathrm{mi}$ east of the 1100 Area, and the Yakima River, about $2 \mathrm{mi}$ to the west. Both streams are important sources of industrial, agricultural, and domestic water for the region. Other streams in the vicinity of the 1100 Area are ephemeral. No surface water or ephemeral streams are present within the 1100 Area or the Horn Rapids Disposal. The area closest to the 1100 Area that has potential for becoming an ephemeral stream lies approximately $3900 \mathrm{ft}(1200 \mathrm{~m})$ west of the Battery Acid Pit at the 1171 Building.

Mean annual precipitation within the Pasco Basin ranges from less than 7 in. $(18 \mathrm{~cm})$ within the Hanford Site to a maximum of $15 \mathrm{in} .(38 \mathrm{~cm})$ atop Rattlesnake Mountain. Total annual precipitation over the entire basin is estimated to be approximately 800,000 acre-ft $\left(9.9 \times 10^{8} \mathrm{~m}^{3}\right)$, with an average 
annual precipitation of less than 8 in. $(20 \mathrm{~cm})$. Mean annual runoff is generally less than $0.5 \mathrm{in} .(1.3 \mathrm{~cm})$ for most of the basin (Leonhart 1979).

Average annual pan evaporation exceeds $60 \mathrm{in.}(152 \mathrm{~cm})$. Average annual lake evaporation ranges from 39 to $41 \mathrm{in.}(100$ to $105 \mathrm{~cm})$. Actual evapotranspiration is essentially equivalent to annual precipitation (Leonhart 1979). Each of the individual waste sites in the 1100 Area is characterized by interior drainage, such that significant surface runoff is unlikely. 


\subsection{PLAN FOR INSTALLATION AND SAMPLING OF WELLS}

OBJECTIVES AND APPROACH

This plan has been developed to guide the construction and initial sampling of five monitoring wells near the 1100 Area and Richland water supply wells.

objectives

The objective of this project is to install five monitoring wells between the 1100 Area waste disposal sites and the Richland water supply wells to provide information on:

- hydrogeologic characterization

- direction of ground-water flow between the waste sites and the water supply wells

- ground-water quality between the waste sites and the water supply wells.

The water supply wells of concern include the Duke Wells, the North Richland Well Field, and well 30000-5 (Figure A.2).

Approach

The project approach includes the following:

- locate new well sites using specific criteria

- construct the wells using previously proven Resource Conservation and Recovery Act (RCRA) design

- collect ground-water samples from the wells and analyze them for potential contaminants Tisted in Table 3.1 . 
TABLE 3.1. Constituents for which Samples Collected from Wells in the Vicinity of the 1100 Area Will Be Anatyzed

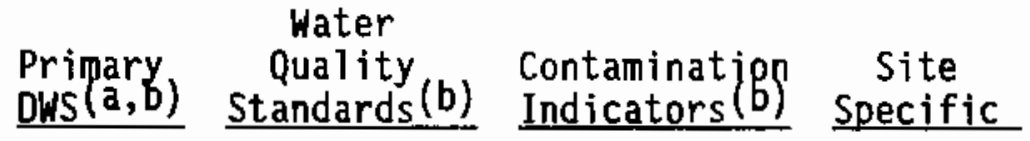

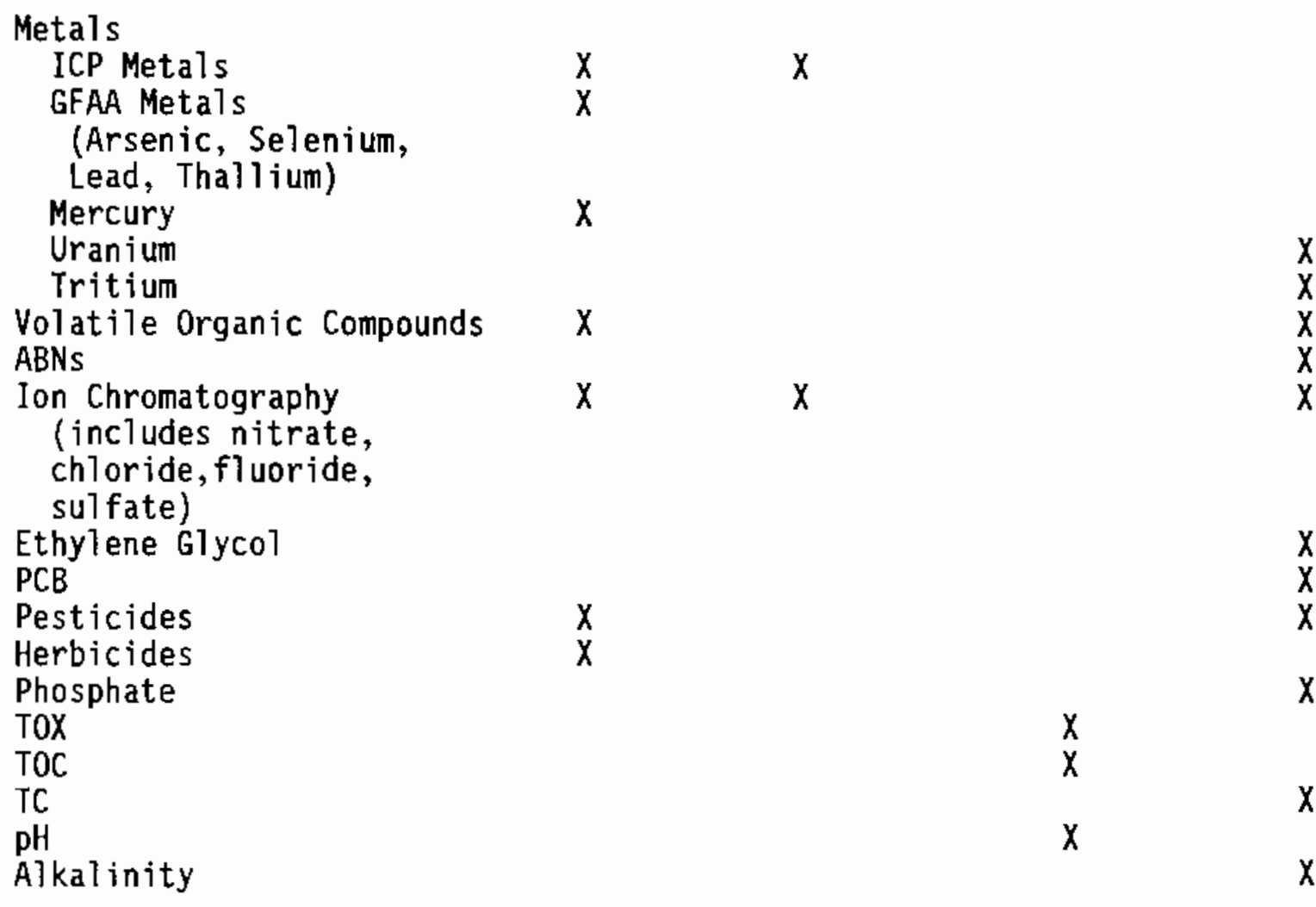

(a) EPA Interim Primary Drinking Water Standards.

(b) EPA 1987, 40 CFR 265.

\section{PLACEMENT OF THE FIVE MONITORING WELLS}

The following criteria have been identified as important to the location of the new wells:

- Location

- must be located between the known waste disposal sites and the Richland water supply wells

- must be located closer to the water supply wells than to the waste sites 
- must consider spatial distribution of existing monitoring wells

- must consider ground-water recharge sources

- must provide DOE access to the well location

- must consider safety issues, such as the location of power lines

- must consider the anticipated ground-water flow direction, both natural and artificial.

- Depth

- must be completed in the same hydrogeologic horizon as the Richland water supply wells

- must consider stratigraphy as indicated by existing we1]s

- must use conservative estimates of completion depth to ensure sufficient we1l construction materials are on site to prevent any delays in completion of the wells.

Four wells will be completed to allow ground water to be sampled for contaminants that tend to remain in the upper portion of the aquifer. One well will be completed to allow sampling of contaminants that tend to sink to the bottom of the aquifer.

Figure A.2 identifies the location of known waste disposal sites, Richland water supply wells, and existing ground-water monitoring wells in the vicinity of the 1100 Area. Sources of ground-water recharge include the recharge basins in the North Richland Well field, disposal of waste water at the Lamb Weston potato processing piant, and irrigation of agricultural land and 1 awns in and north of Richland.

The location of power 1 ines will be examined in the field when the well locations are staked. The location of other utilities will be determined by Westinghouse Hanford Company before the initiation of drilling. Well locations are not expected to be significantly affected by existing utilities.

The direction of ground-water flow is not completely understood in the area between the 1100 Area waste sites and the Richland water supply wells. 
The regional flow is generally from west to east in response to the differences in elevation between the Yakima and the Columbia rivers. The Yakima River is approximately $370 \mathrm{ft}(113 \mathrm{~m})$ above mean sea level, and the Columbia River in the area of interest is approximately $340 \mathrm{ft}(104 \mathrm{~m})$ above mean sea levet.

Operation of the recharge basins and the water supply wells in the area will probably have a significant effect on the water table configuration and on the direction of ground-water flow. At certain times of the year, the recharge basins within the North Richland Well Field probably create a significant recharge mound causing ground water to flow away from the well field and around it to the north and south in response to the regional ground-water flow.

The locations of the five new wells are shown in Figure A.5. Wells 1 and 3 are located between the North Richland Well Field and two waste disposal sites. The two sites are the Battery Acid Pit and the site of the exhumed waste Antifreeze Tank. The Battery Acid Pit received an estimated 15,000 gal $(57,000$ liters) of battery acid and is considered to have the highest potential impact on the quality of the ground water. The wells are located some distance from the recharge ponds to increase the chance that representative ground water (i.e., unaffected by the recharge ponds) will be collected. This location will also provide water level elevation data that will help determine the direction of ground-water flow between the waste sites and the water supply wells. Well 1 will be completed at the top of the aquifer to intercept any potential contaminants there; Well 3 will be completed near the bottom of the aquifer to intercept contaminants that have migrated to that depth.

We11 2 is located close to the North Richland Well Field and midway between the Battery Acid Pit and Antifreeze Tank to the south and the Paint and Solvent Site and Antifreeze and Degreaser Site to the north. This well will be completed at the top of the aquifer because potential contaminants are most likely to be found there and the water supply wells of the North Richland Well field tap the top of the aquifer. This location will provide 
water level elevation data that may be used to help understand the impact of the North Richland Well Field (recharge/discharge) on the local ground-water flow system.

Well 4 is located south of the other wells between the waste disposal sites and the Duke Wells. During periods when a ground-water mound exists beneath the recharge ponds in the North Richland Well Field, ground water may flow south from the vicinity of the Battery Acid Pit and the Antifreeze Tank toward the Duke Wells. Data from a well at this location will assist in determining if flow to the south does occur.

Well 5 is located north of the well field and will be used to sample ground water in the vicinity of well 3000D-5. The ideal location for this well is between well 3000D-5 and the Paint and Solvent Site and Antifreeze and Degreaser Site; however, the 1and to the west of 3000D-5 is not accessible to DOE. The well is located to the north of the water supply well of interest because we 11 3000D-1 and 3000-G are south of the well and disposal sites, and are available for sample collection.

Figure A.6 presents the dri1lers log for wel1 1199-541-13C (3000D-1). This well was drilled in the 1940s and is located near the planned new well locations (Figure A.5). Based on this information, the anticipated hydrogeologic conditions at the planned well sites are summarized as:

- unsaturated sand and gravel (undifferentiated surficial deposits, Hanford formation, and Ringold Formation) to a depth of about $55 \mathrm{ft}(17 \mathrm{~m})$

- water-bearing sand and gravel with some boulders (undifferentiated Hanford and Ringold formations) from about $55 \mathrm{ft}$ to $85 \mathrm{ft}$ (17 to $26 \mathrm{~m}$ )

- silt unit ("lower blue clay member" of the Ringold Formation) from about $85 \mathrm{ft}(26 \mathrm{~m})$ to a total depth of $95 \mathrm{ft}(29 \mathrm{~m})$.

The water supply wells of the North Richland Well Field are screened over approximately the upper $40 \mathrm{ft}(12 \mathrm{~m})$ of the unconfined aquifer. 
Table 3.2 contains recommended well depths and screened intervals based on the available hydrogeologic information and the completion of the water supply wells.

\section{Drilling and Well Installation}

Detailed information on the functional design and construction standards for the four shallow and one deep monitoring wells is presented in Appendix $C$ and is intended to be consistent with HS-V-50005 (Generic Construction Specifications for Drilling Ground Water Monitoring Wells at RCRA Sites on the Hanford Reservation). The materials and methods of installation meet the WAC-173-160 (Ecology 1988) requirements for resource protection wells. The wells will be constructed with a cable-tool drill using the drive-barrel technique where possible and the hard-tool technique where necessary. To prevent introduction of contaminants into the borehole, the drill rigs and associated equipment will be steam-cleaned before drilling each new well.

Temporary carbon steel casing will be used to keep the well open and to seal off any contamination that may be encountered during drilling. The minimum inside diameter of the temporary casing will be $8 \mathrm{in} .(20 \mathrm{~cm})$ to allow enough room for the permanent 4 -in $(10-\mathrm{cm})$. stainless steel casing. None of the temporary carbon steel casing will be left in the borehole after completion.

IABLE 3.2. Recommended Well Depths and Screened Intervals

$\begin{array}{ccc}\begin{array}{c}\text { We11 } \\ \text { Number }\end{array} & \begin{array}{c}\text { Tota] } \\ \text { Depth } \\ \text { (ft) }\end{array} & \begin{array}{c}\text { Screen } \\ \text { Length } \\ \text { (ft) }\end{array} \\ \text { Well 1 } & 75 & 15 \\ \text { We11 2 } & 75 & 25 \\ \text { We11 3 } & 130 & 10 \\ \text { Wel1 } 4 & 75 & 15 \\ \text { Wel1 5 } & 75 & 15\end{array}$


After reaching the desired depth, the borehole will be logged (natural gamma). Water samples will be collected after first reaching the water table and rapidly analyzed to determine if surface disposal of the purge water is acceptable. Purge water will be analyzed for the following constituents to determine appropriate disposal:

- lead

- ethylene glycol (antifreeze)

- volatile organic acids

- aipha radiation

- beta radiation

- gamma radiation.

After the borehole has been completed, the permanent stainless steel casing will be set, and the well completion process will begin. The planned well will use flush-joint threaded, 4-in.-dia (10-cm-dia), stainless steel casing and screen. After the permanent casing has been set, all temporary carbon steel casing will be pulled as the filter pack or annular seal material is added. At least $2 \mathrm{ft}(0.6 \mathrm{~m})$ of filter pack or seal material must be kept inside the temporary casing, both to maintain the integrity of the borehole and to ensure adequate placement of the filter pack and seal. The selected silica sand filter pack will be placed in the annulus from total well depth to at least $3 \mathrm{ft}(1 \mathrm{~m})$ above the top of the screen. A $3-$ to $5-\mathrm{ft}$ (1- to $1.5-\mathrm{m}$ ) thick bentonite-pellet seal will then be placed on top of the sand pack. The annulus above the bentonite pellets and up to approximately $20 \mathrm{ft}(6 \mathrm{~m})$ below ground surface will be filled with bentonite (crumbles, granules, or slurry). Air-entrained Portland cement will then be installed to $3 \mathrm{ft}$ below ground surface. After all the temporary carbon steel casing has been removed, a $4-\mathrm{ft}(1.2-\mathrm{m})$ by $4-\mathrm{ft}(1.2-\mathrm{m})$ by $6-\mathrm{in} .(15-\mathrm{cm})$ thick concrete pad will be poured and a $6-i n .(15-\mathrm{cm})$ stainless steel protective casing installed around the permanent casing. A brass survey marker will be set in the concrete to record the well name. Guardposts will be installed at each corner of the pad to protect the well head. Schematic diagrams of a completed shallow and deep well are shown in Figures $A .7$ and A.8, respectively. The wells will be developed by bailing and/or pumping. Water removed from the well during development will be disposed of in accordance 
with an approved effluent monitoring plan. After development, a HydroStan(a) pump will be placed in the well to facilitate sample collection. A locking well cap will be used to restrict access to the well and protect the aquifer.

After completion, the monitoring well will be surveyed for location and elevation by a licensed surveyor. The elevation of the brass marker and the top of the stainless steel casing will be determined to the nearest $0.01 \mathrm{ft}$ $(3 \mathrm{~mm})$, with a mark placed on each to indicate the location that was surveyed. The areal location will be determined to the nearest $0.5 \mathrm{ft}(15 \mathrm{~cm})$. All horizontal measurements will be referenced to Lambert Coordinates, and all vertical measurements will be referenced to the National Coast and Geodetic Survey makers.

\section{HYDROGEOLOGIC CHARACTERIZATION}

This section presents the types of samples and analyses that will be performed during and after well installation. The resulting data will then be used to describe the geologic and hydrologic features of the area in the vicinity of the 1100 Area and the Richland water supply wells. Work will be conducted following established procedures presented in the appendices.

Geologic Characterization

Data collected during the drilling of monitoring wells will be used to characterize the geology and stratigraphy of the area. Types of data to be collected are discussed below and in Appendix D.

\section{Geologic Sampling}

Two 1-pint (0.5-1iter) drilling samples will be collected at 5-ft (1.5-m) intervals or at changes in 1ithology. Samples are to be collected with a drive barrel in the unsaturated sediments. If drilling with a drive barrel becomes ineffective, the driller may switch to the hard tool, and samples will be collected with a bailer. Moisture samples will be collected every $5 \mathrm{ft}(1.5 \mathrm{~m})$ in the unsaturated sediments when using the drive barrel.

(a) Hydrostar is a trademark of Instrumentation Northwest, Incorporated, Redmond, Washington. 
Sediments that are contaminated and classified as radioactive or as hazardous waste will be handled, transported, and disposed of in accordance with existing Westinghouse Hanford Company procedures.

The samples collected will be described as hand specimens in the field and documented on drill logs by the site geologist. Selected samples will be collected for laboratory analyses of mojsture content. These activities will be performed in accordance with the procedures and guidances found in Last and Liikala (1987).

The borehole and samples will be monitored for radiation on a regular basis. The site will be tested for contamination periodically and at a minimum of once per day with radiation detection instruments, a photo-ionization detector, Drager tube, or gas chromatograph, depending on the type of contamination anticipated. Necessary equipment and instructions for dealing with the particular contamination anticipated will be covered in the site safety plans. Samples of sediment that is suspected of contamination will be tracked using chain-of-custody procedures.

If perched water is encountered, it will be checked by a radiation protection technician and sampled for analysis, and the zone also will be cased off or grouted to prevent migration.

\section{Sample Analyses}

Selected samples will be collected for 1aboratory analyses of moisture content. These analyses will be performed on drive-barrel samples collected from the vadose zone.

\section{Borehole Logging}

The borehole will be geophysically logged using the natural gamma logging tool. Logs will be annotated to show casing location and sizes. At completion, each well will also be viewed with the aid of a down-well video camera to determine if the well is acceptably clean and undamaged.

\section{Data Interpretation and Presentation}

All geologic and geophysical data will be interpreted by geologists to determine the stratigraphy beneath the site. These data will be presented in 
cross sections, fence diagrams, and tables as recommended in the RCRA GroundWater Monitoring Technical Enforcement Guidance Document (TEGD) (EPA 1986).

The data, interpretations, and recommendations will be presented in a letter report to Westinghouse Hanford Company. The report will include 1) as-built drawings, 2) inspection reports, 3) borehole geologic logs, 4) preliminary cross sections, 5) laboratory results, 6) sampling and analysis results (soil and water), and 7) borehole survey results.

Sampling and Analys is

Sample collection techniques and control, analytical methods, and quality assurance/quality control are presented in this section. A specific list of monitoring parameters is presented in Table 3.1.

Sample Collection. Preservation, and Handling

Ground-water quality sampling will be conducted for each of the five new wells. Each well will be equipped with a dedicated Hydrostar pump, capable of pumping 3 to $5 \mathrm{gal} / \mathrm{min}$ (11 to 19 liters/min). Ground-water samples will be collected by trained personnel from Pacific Northwest Laboratory (PNL) following procedures presented in Appendix $E$. These procedures were deve1oped from established Hanford-DOE procedures and TEGD guidelines (EPA 1986), and specify the type of sample bottle and preservation requirements for each sample type and the chain-of-custody procedures controlling the integrity of each sample from the time of field collection through analysis.

Water levels are to be measured before each well is sampled. After measurement, each well should be purged a minimum of three well-bore volumes or until the well is thoroughly flushed of standing water to obtain a representative sample of the formation water. Indicator parameters (e.g., temperature, pH) will be monitored before sample collection to ensure the sample is representative of aquifer conditions.

The ground-water samples will be analyzed for the parameters listed in Table 3.1. Analyses are performed at United States Testing Company (UST). 


\section{Quality Assurance/Quality Control}

Quality assurance (QA) for the initial sampling of the new wells will be conducted in accordance with PNL's quality assurance manual. A QA plan describing the manner in which specific QA requirements are to be met has been prepared for the site-wide Ground-Water Monitoring Project. This plan will be followed for documenting the construction and sampling of the five new wells and is presented in Appendix F.

Quality control (QC) will be conducted in accordance with the PNL technical procedure P-7, as presented in Appendix E, and UST's internal QC program. The purpose of this effort is to determine and document the quality of the analytical results being produced by the laboratory and to institute corrective actions as needed. The QC effort has two main components:

1) routine internal checks performed by UST, and 2) external checks conducted by PNL to independently evaluate UST performance. Project samples will be submitted to the analytical laboratory in the same way that samples from the Site-Wide Ground-Water Monitoring Project are submitted and so can be evaluated based on the QC information generated by the Site-Wide project. The scope of these efforts is described in the following sections.

Laboratory Quality Control. Internal QC at UST includes general practices applicable to a wide range of analyses, as well as specific procedures stipulated for particular analyses. The QC and QA programs at UST are documented in the UST Quality Control Manual (Hembree et a1. 1986) and the Quality Assurance Manual (Hembree and Lardy 1986). UST produces a quarterly Quality Control Report of Hazardous Substance Analyses to PNL for review by personnel in subcontracts, sample analyses management, and QC, and statistical task leaders of the ground-water monitoring program.

Field/External Quality Control. Pacific Northwest Laboratory will use both interlaboratory comparisons and spiked, replicate, and blank samples in evaluating the accuracy of results from UST. The purpose and scope of each of these are as follows.

Interlaboratory comparisons using field samples are conducted to determine if the results obtained by the primary laboratory, UST, are comparable to those obtajned from other laboratories. Comparisons are currently being 
conducted for anions, volatile organic compounds, and metals. Each month, replicate samples from selected wells are delivered to four different PNL laboratories. The results from these PNL laboratories are then compared with the results from UST.

Spiked samples are submitted to UST to estimate the bias of analytical laboratory procedures and to determine when this bias exceeds control limits. Two types of spiked samples are being used: 1) blind, spiked samples prepared by PNL, and 2) spiked samples prepared under an established multilaboratory comparison program. BTind, spiked samples containing metals, anions, herbicides, pesticides, and volatile organic compounds (e.g., halomethanes) have been submitted quarterly since January 1986. These samples were prepared by PNL with materials supplied by Environmental Resource Associates. Additional spiked samples prepared with materials supplied by the U.S. Environmental Protection Agency (EPA) were added in June 1986. The constituents included are ammonium ion, cyanide, acid/base/neutral (ABN) compounds, and an expanded number of pesticides and volatile organic compounds. The performance of various analyses, including analyses for semivolatile organic compounds, thiourea, phosphoric pesticides, ethylene glycol, sulfide, and perchlorate, will be monitored. United States Testing Company has also received and analyzed spiked samples submitted under the EPAsponsored Water Pollution Laboratory Performance Evaluation Study WP016 and Water Supply Laboratory Performance Evaluation Study WSO18.

Replicate analyses of field samples will be conducted to establish how much variability might be expected in the laboratory measurements performed on nearly identical samples. Trip blanks will be submitted to UST to determine whether environmental conditions encountered during collection and transportation of samples have affected the results obtained by analysis. One trip blank is submitted each sample period per area at the rate of one for 1 to 20 wells. The trip blank is analyzed for volatile organic compounds.

Quality Control Documentation. Relevant information concerning analysis of the samples will be entered into logbooks that are maintained for each analytical procedure. This information might include calibration data, 
sample data, instrument conditions, matrix spike recoveries, and standards preparation. Other data needed to support sample results, such as chromatograms, charts, and tables, will be labeled and stored in a secure area.

Sample data generated by the analysts at the UST Hazardous Substance Analysis Laboratory are entered into UST's main computer by a data handler. The data sheets provided by the analysts for this purpose will include

- sample identification

- sample results in appropriate units

- extraction date (if any)

- analysis data

- dilutions (if any)

- matrix spike recoveries

- reagent blank results

- any other QC results.

Copies of the analysts' data sheets will also be provided to the 1aboratory supervisor or director for review.

Data are entered in accordance with written procedures (UST 1986), and discrepancies or anomalies are resolved at this time. For all analyses, except total organic halogens (TOX), total organic carbon, and radionuclides, results less than the contractual detection limits are entered as equal to the detection limit. For those stated exceptions, the actual results are entered into the computer. During data entry, an asterisk is automatically added by the computer to all results at or below the detection limit.

After all data for a sample set have been entered, an internal report is generated to verify that the data have been correctly entered. If corrections are needed, a second report is generated for verification. Finaliy, the data are copied onto a magnetic tape in the format specified by PNL. A hard-copy summary report and data file listing are generated and delivered to PNL with the tape and copies of the analysts' data sheets. Deliveries of data to PNL are generally made once per week. 



\subsection{REFERENCES}

Bjornstad, B. N. 1985. "Late-Cenozoic Stratigraphy and Tectonic Evolution within a Subsiding Basin, South-Central Washington." Geological Society of America Abstracts with Programs 17:524.

Caggiano, J. A., and D. W. Duncan (eds.). 19B3. Preliminary Interpretation of the Tectonic Stability of the Reference Repository Location, Cold Creek Syncline, Hanford Site. RHO-BW-ST-199, Rockwe11 Hanford Operations, Richland, Washington.

DOE. 1986. Reference Repository Location, Hanford Site, Washington. Vol. 2, D0E/RW-0070, U.S. Department of Energy, Richland, Washington.

DOE. 1988. Consultation Draft of the Site Characterization Plan. Vol. 1, U.S. Department of Energy, Richland, Washington.

Ecology. 1988. "Minimum Well Construction Standards." Washington Administrative Code, Chapter 173-303-160, Washington State Department of Ecology, Olympia, Washington.

EPA. 1986. RCRA Groundwater Monitoring Technical Enforcement Guidance Document (TEGD). OWSER-9950.1, U.S. Environmental Protection Agency, Washington, D.C.

EPA. 1987. "Interim Status Standards for Owners and Operators of Hazardous Waste Treatment, Storage, and Disposal Facilities." U.S. Code of Federal Regulations. 40 CFR 265.

Fecht, K. R., S. P. Reidel, and A. M. Taliman. 1985. Paleodrainage of the Columbia River System on the Columbia Plateau of Washington State: A Summary. RHO-BW-SA-318P, Rockwe11 Hanford Operations, Richland, Washington.

Gee, G. W. 1987. Recharge at the Hanford Site: Status Report. PNL-6403, Pacific Northwest Laboratory, Richland, Washington.

Graham, M. J., M. D. Hall, S. R. Strait, and W. R. Brown. 1981. Hydrology of the Separations Areas. RH0-ST-42, Rockwell Hanford Operations, Richland, Washington.

Hembree, N. H., and M. M. Lardy. 1986. Quality Assurance Manual. Rev. 6, U.S. Testing Company, Inc., Richland, Washington.

Hembree, N. H., M. M. Lardy, and R. G. Swoboda. 1986. Quality Control Manual. Rev. 3, UST-R-QA-9-80, U.S. Testing Company, Inc., Richland, Washington.

ICF. 1987. Hydrogeologic Study of North Richland Well Field and Groundwater Recharge Basins: A Report Prepared for the City of Richland, Washington. ICF Northwest, Richland, Washington. 
Last, G. V., and T. L. Liikala. 1987. A Field Guide for Well Site Geologists: Cable Tool Dri]ling. PNL-6392, Pacific Northwest Laboratory, Richland, Washington.

Leonhart, L. S. 1979. Surface Hydrologic Investigations of the Columbia Plateau Region. Washington. RHO-BWI-ST-6, Rockwell Hanford Operations, Richland, Washington.

Lindberg, J. W., and F. W. Bond. 1979. Geohydrology and Groundwater Quality Beneath the 300 Area, Hanford Site, Washington. PNL-2949, Pacific Northwest Laboratory, Richland, Washington.

Newcomb, R. C. 1958. "Ringold Formation of Pleistocene Age in Type Locality, The White Bluffs, Washington" American Journal of Science $256: 328-340$.

Newcomb, R. C., J. R. Strand, and F. J. Frank. 1972, Geology and Groundwater Characteristics of the Hanford Reservation of the U.S. Atomic Energy Commission, Washington. United States Geological Survey Professional Paper 717, United States Government Printing Office, Washington, D.C.

PSPL. 1982. Skaqit/Hanford Nuclear Project, Application for Site Certification/Environmental Report. Vo1. 2, Chapters 4-8, Puget Sound Power and Light Company, Bellevue, Washington.

Reidel, S. P., K. R. Fecht, and R. W. Cross. 1982. "Constraints on Tectonic Models for the Columbia Plateau from the Age and Growth Rates of Yakima Folds." Proceedings of the 33rd Alaska Science Conference, Arctic Division. American Association for Advancement of Science, Vol. 12.

Reidel, S. P., G. R. Scott, D. R. Bazard, R. W. Cross, and B. Dick. 1984. "Post-12 Million Year Clockwise Rotation in the Columbia Plateau, Washington." Tectonics 3(2):251-273.

Schalla, R., R. W. Wallace, R. L. Aaberg, S. P. Airhart, D. J. Bates, J. V. M. Carlile, C. S. Cline, D. I. Dennison, M. D. Freshley, P. R. Heller, E. J. Jensen, K. B. 01 sen, R. G. Parkhurst, J. T. Rieger, E. J. Westergard. 1988. Interim Characterization Report for the 300 Area Process Trenches. PNL-6716, Pacific Northwest Laboratory, Richland, Washington.

Supply System. 1981. Final Safety Analysis Report WPPSS Nuclear Project No. 2. Amendment 18, Washington Public Power Supply System, Richland, Washington.

Swanson, D. A., T. L. Wright, and I. Zietz. 1979. Aeromagnet ic Map and Geological Interpretation of the Western Columbia Plateau, Washington and Adjacent Oregon. Open-File Report 76-51, Map GP-917m, U.S. Geological Survey, Denver, Colorado. 
Tolan, T. L. 1986. Tectonic Map of the Columbia Plateau and Adjacent Areas Scale 1:50,000. SD-BW1-TI-320, Rockwell Hanford Operations, Richland, Washington.

UST. 1986. Procedure Manual. UST-RD-PM-9-80, U.S. Testing Company, Richl and, Washington. 

APPENDIX A

FIGURES 


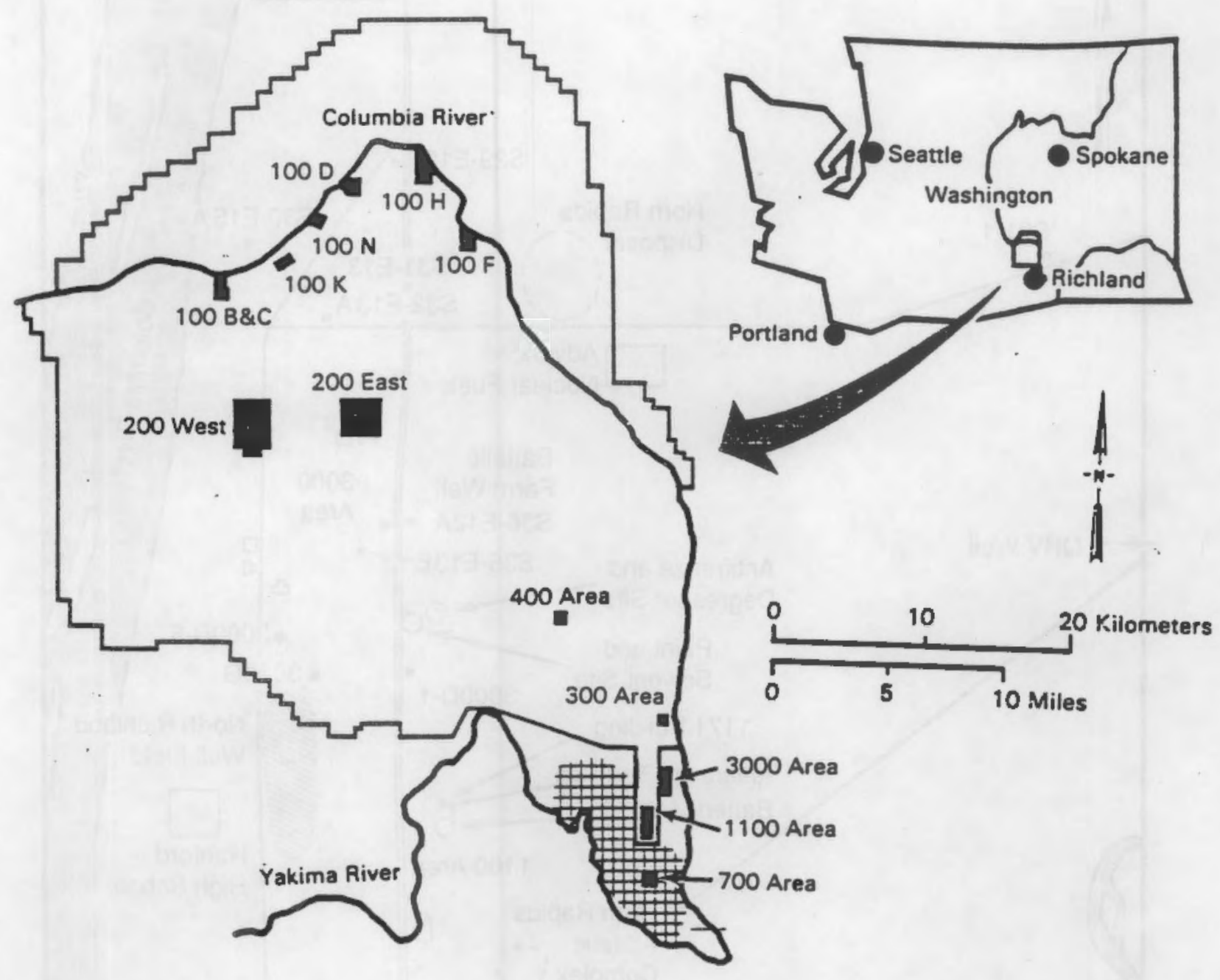

FIGURE A.1. Hanford Site Map 


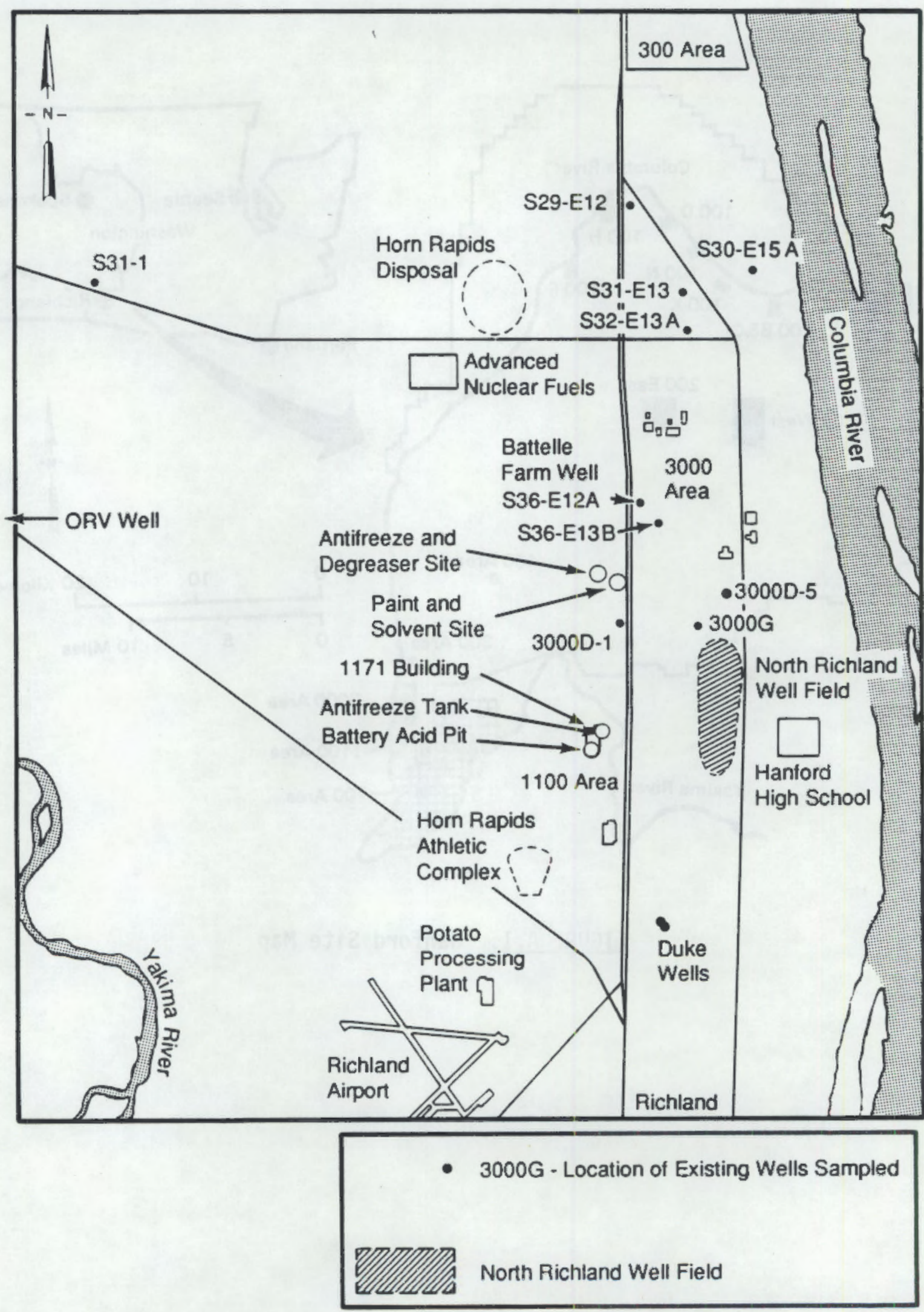

FIGURE A.2. 1100 Area and North Richland Well Field (figure not to scale) 


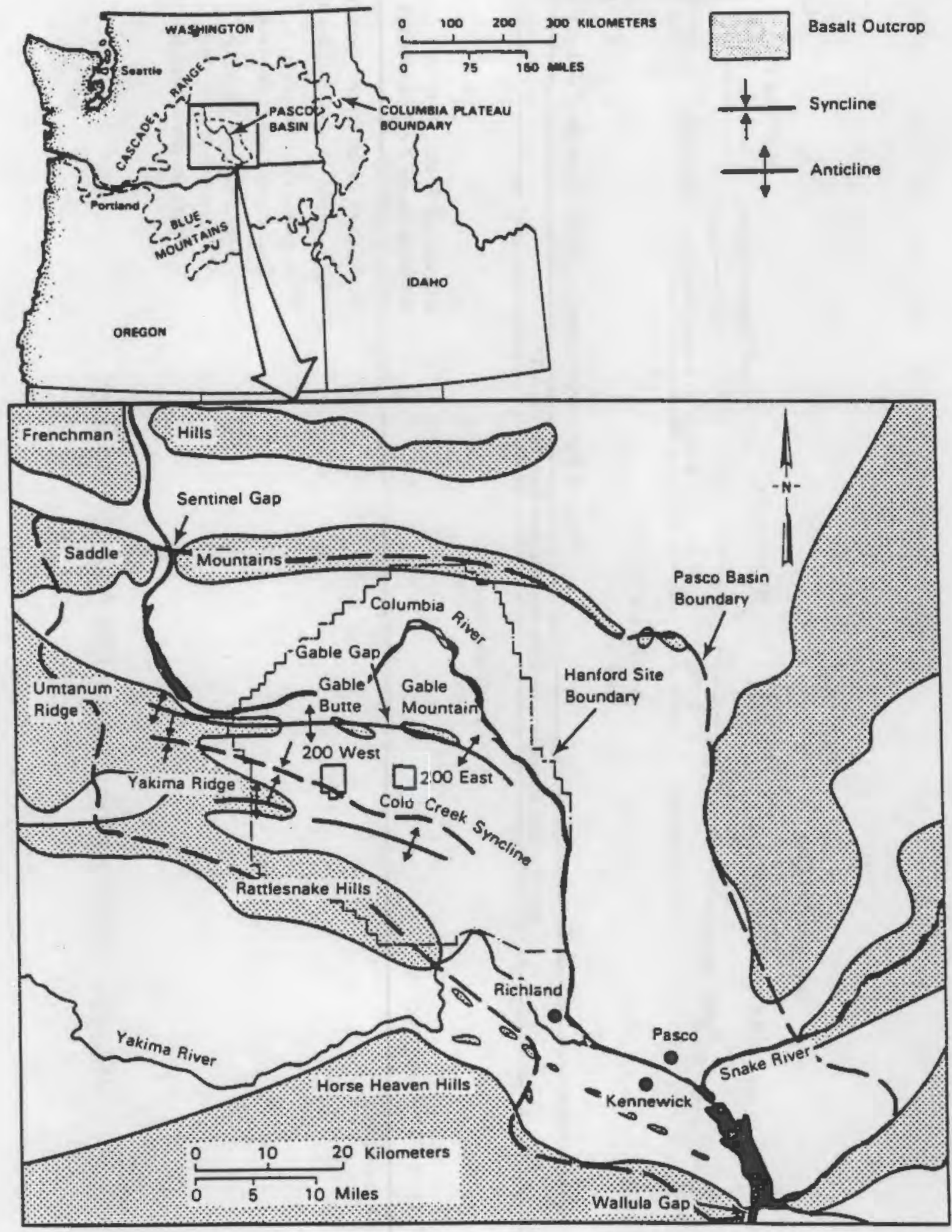

FIGURE A.3. Structural Setting of the Hanford Site (modified from Caggiano and Duncan 1983) 


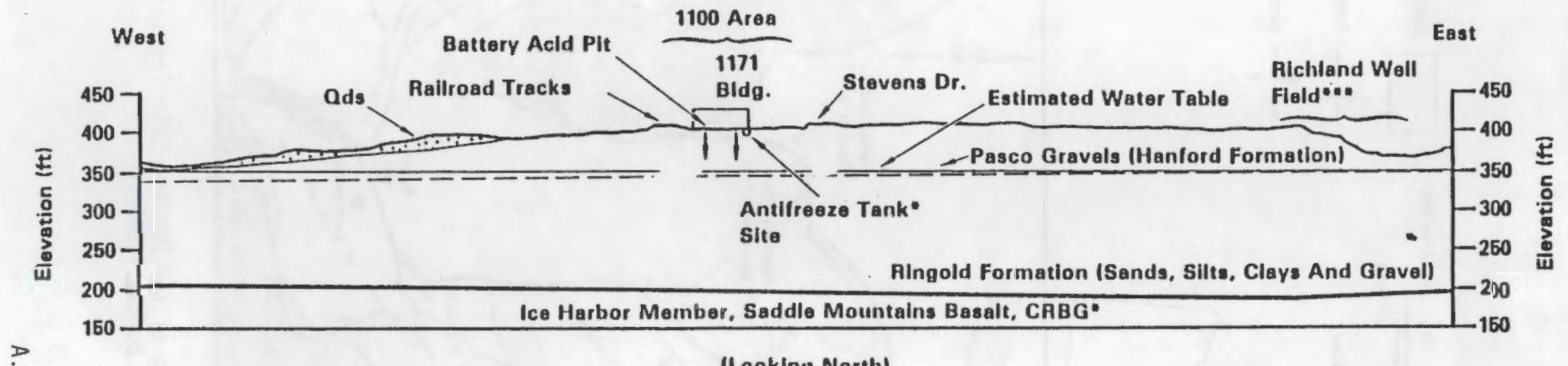

(Looking North)

Horzontal Scals: $1^{\prime \prime}=600^{\circ}$

Qde $=$ Dune Sands

Vertlcal Scale Exaggerated $(3.9 \times)$

- Columbla Rlver Basalt Group

" "Projected 375' To LIne Of Section

- "Hypothetical Location.

Exact Location Unknown.

" "Water Table In Vicinity Of Richland Well Field is Variable In Time And Spece

FIGURE A.4. Cross Section Showing 1100 Area Stratigraphy 


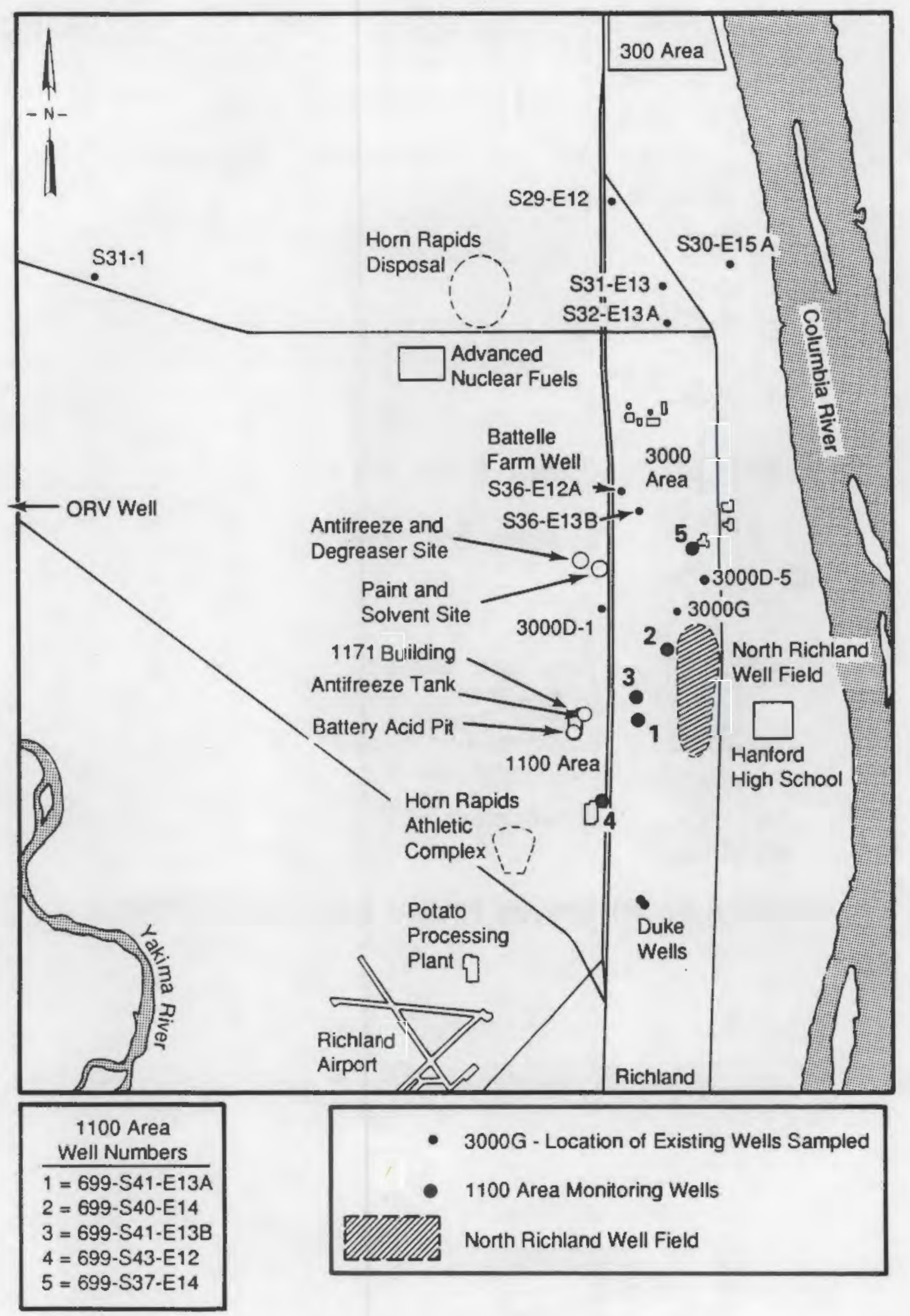

FIGURE A.5. Planned New Well Locations (figure not to scale) 


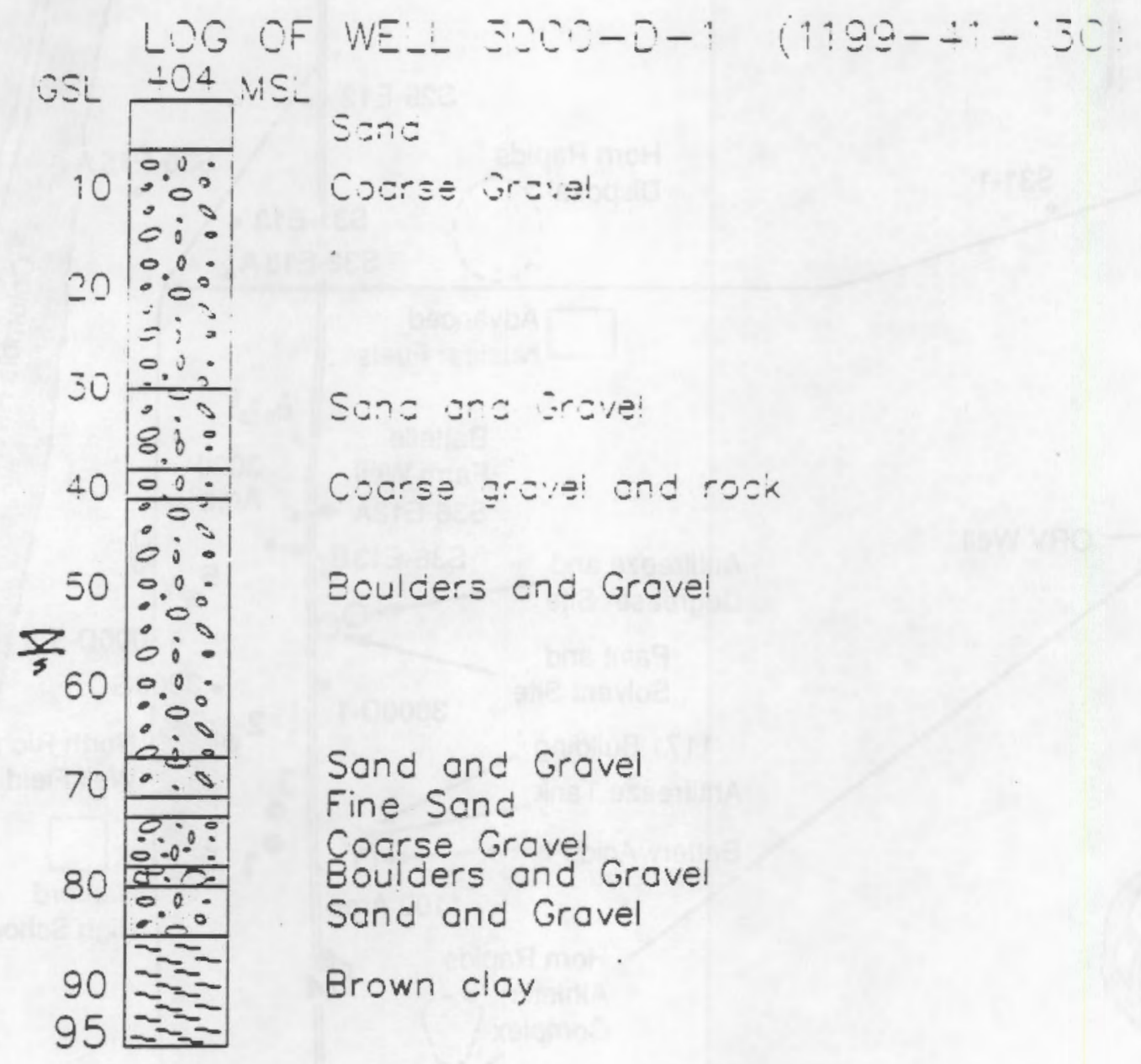

FIGURE A.6. Drillers Log for Well 119-S41-13C (3000D-1) 


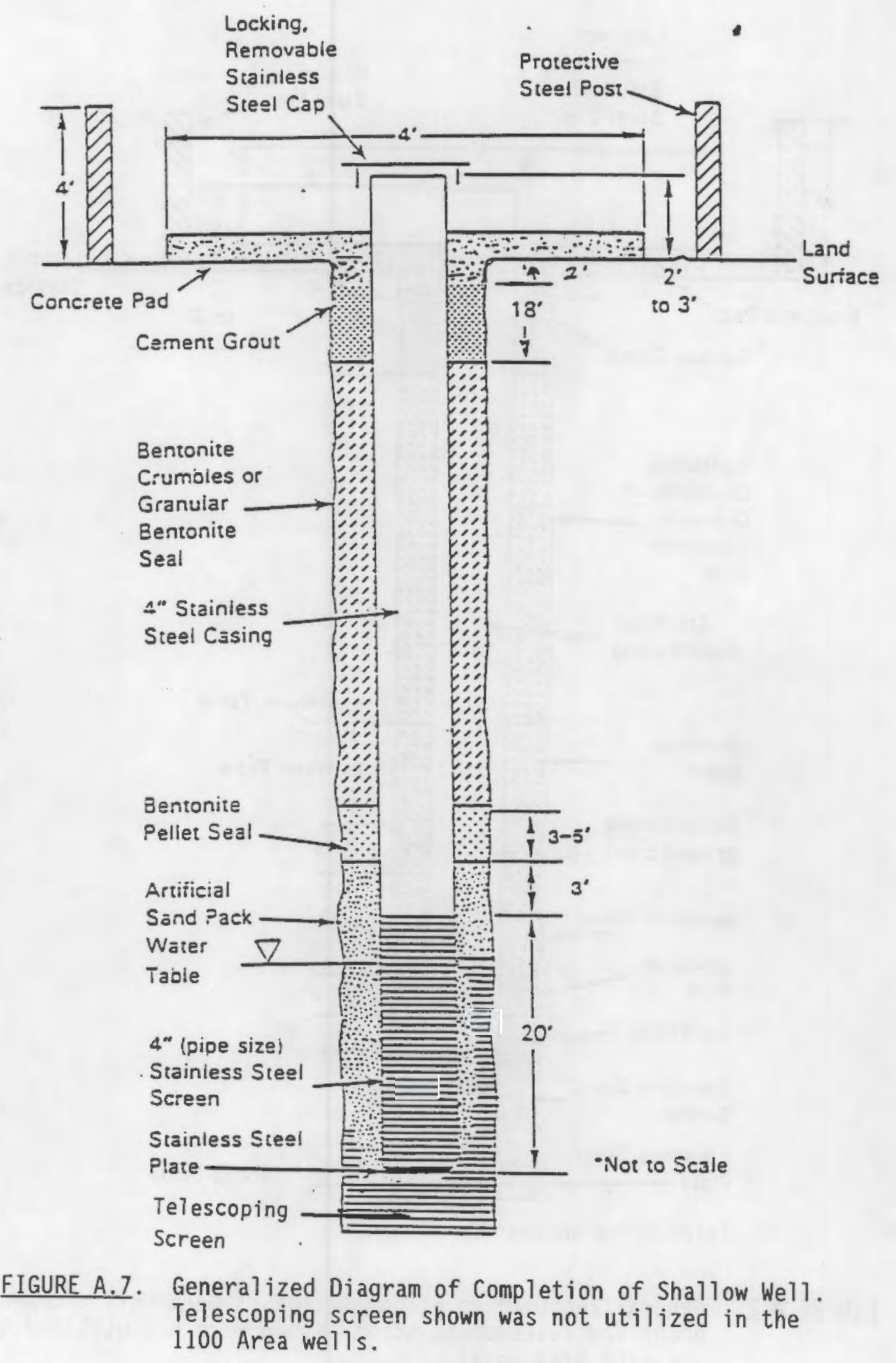

A. 7 


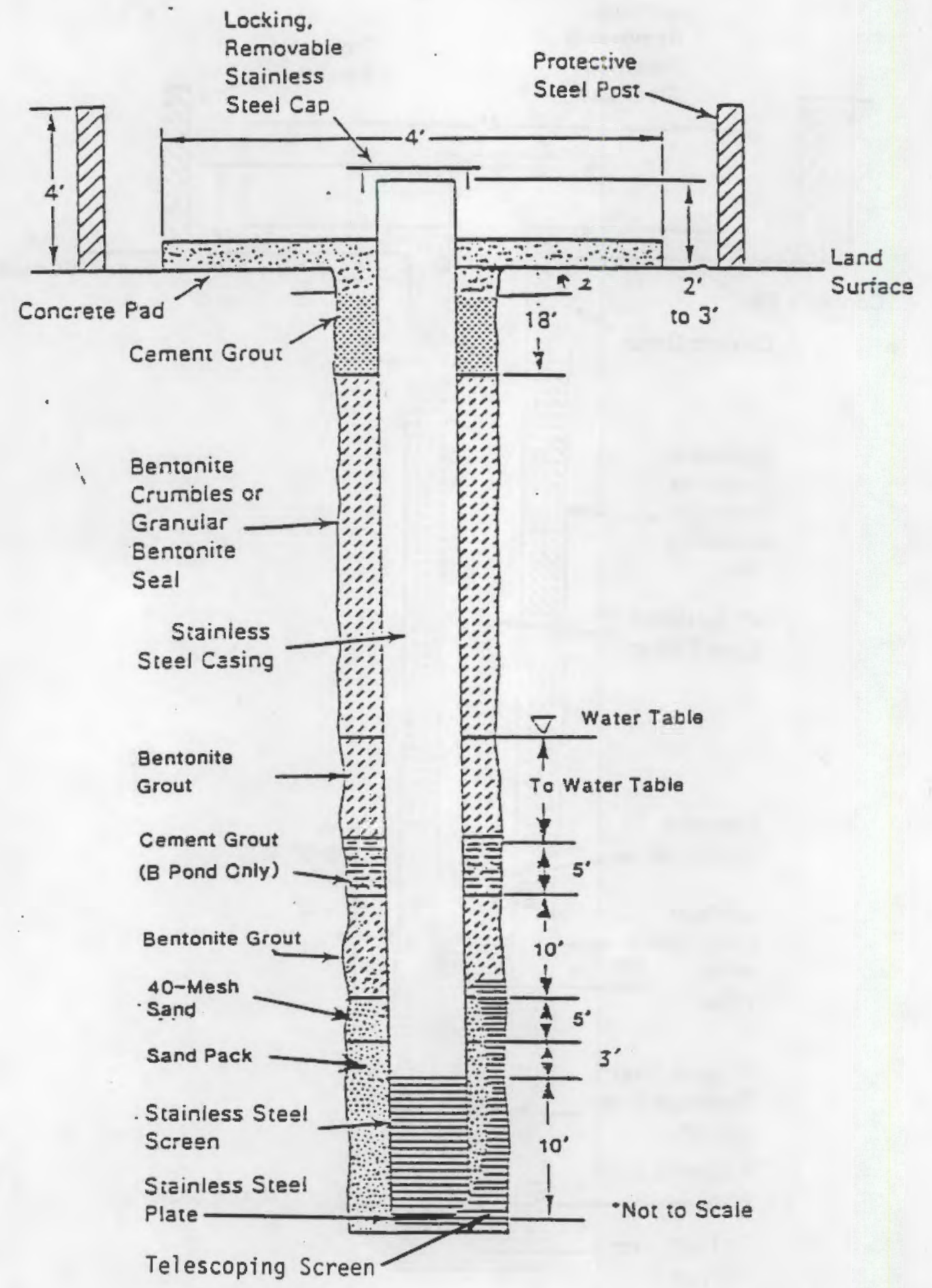

FIGURE A.8. Generalized Diagram of Completion of Deep Well. Cement grout and telescoping screen shown were not utilized in the 1100 Area wells. 
Procedure for Collection and Documentation of Drjliting Data, P-6 for RCRA Compliance/Hazardous Matertals Monitoring

\subsection{OBJECTIVE}

This procedure provides standardized methods for the collection and documentation of sediment sanples and well construction data, for we?ls drilled by the cable tool method.

\subsection{APPLICABILITY}

This procedure appijes to work performed by well site geologists during well construction.

\subsection{RESPONSIBILITIES}

\subsection{Well Site Geologist}

- Collect and document drilling data

\subsection{Senior Technical Reviewer}

- Reyiew and sign/date Well Completion Report/Title III Inspection List

- Reylew and sign/date As-zuilt Diagram

- Reyiey and sign/date Drill Logs

\subsection{PROCEDURE}

4.1 Collection of Sediment Samoles

Sediment samples shall be collected at 5-foot intemrals and changes $4 \pi$ formation by the well stte geologist. All drill cuttings to be samplea shall be collected from the driller in a 5 gallon bucket.

Two pint jars shall then be filled from the bucket. One jar shall be subatited for laboratory analysis and the other retained for arehiving.

A label fndicating well number, depth, date, drilling nethod and initials of the geologist collecting the sample shall be placad on each sanpie jar. Well number, depth and date siall also be written on the lid of each jar.

\begin{tabular}{|c|c|c|c|}
\hline $\begin{array}{l}\text { Approvals: } \\
\text { Project Manag }\end{array}$ & & QA Rep. 12 & $4 y 3 / 6 / 87$ \\
\hline $\begin{array}{l}\text { Procedure No: } \\
\text { P-6 }\end{array}$ & $\begin{array}{c}\text { Revision No: } \\
0\end{array}$ & $\begin{array}{r}\text { Date Issued: } \\
3-16-87\end{array}$ & Page 1 of 3 \\
\hline
\end{tabular}




\section{APPENDIX D \\ DATA COLLECTION AND DOCUMENTATION PROCEDURES FOR \\ MONITORING WELL INSTALLATION}

This appendix lists the procedures to be used during drilling and well construction activities by Pacific Northwest Laboratory in support of the well installation and sampling of 1100 Area environmental monitoring wells.

D. 1 
APPENDIX B

1100 AREA WATER QUALITY AND WASTE SITE DATA 


\section{TABLE B.1. Analytical Results for Surface Soil Samples from the 1100 Area}

\begin{tabular}{|c|c|c|c|c|c|c|c|c|}
\hline COAST I TUENT ( $49 / 9)$ & $\begin{array}{l}\text { Battery } \\
\text { Acid Pit } \\
(1100-1) \\
\text { BAP001A01 }\end{array}$ & $\begin{array}{l}\text { Battery } \\
\text { Acid Pit } \\
\text { (t100-1) } \\
\text { Bap001B01 }\end{array}$ & $\begin{array}{l}\text { Spill lest } \\
\text { of Tracks } \\
\text { suT001A01 } \\
\end{array}$ & $\begin{array}{l}\text { Asphelt } \\
\text { Emulsion } \\
\text { AEP001A01 }\end{array}$ & $\begin{array}{l}\text { Paint and } \\
\text { solvent Pjt } \\
(1100-2) \\
110002 \mathrm{A01} \\
\end{array}$ & $\begin{array}{l}\text { Paint and } \\
\text { Solvent PIt } \\
(1100-2) \\
110002801 \\
\end{array}$ & $\begin{array}{l}\text { Antifreeze } \\
\text { and } \\
\text { Degreaser } \\
\text { Pit } \\
(1100-3) \\
110003 \mathrm{AO} 1 \\
\end{array}$ & $\begin{array}{l}\text { Antifreeze } \\
\text { and } \\
\text { Degreaser } \\
\text { Pit } \\
(1100-3) \\
190003 \mathrm{~B} 01 \\
\end{array}$ \\
\hline $\mathrm{ALPHA}(\mathrm{pCi} / \mathrm{L})$ & $<1.4$ & 5.3 & 4.2 & 3.9 & 4.3 & 2.6 & $Q .2$ & 4.9 \\
\hline$B E T A(P C i / L)$ & 18.1 & 20.9 & 17.3 & 20.5 & 16.7 & 16.8 & 15.3 & 14.0 \\
\hline $\mathrm{Hg}$ & 1.37 & $<0.2$ & $<0.2$ & $<0.2$ & $<0.2$ & $\infty .2$ & $<.2$ & $\infty .2$ \\
\hline Be & $<0.5$ & $\infty .5$ & $<0.5$ & $\infty .5$ & $<0.5$ & $<0.5$ & 4.5 & $\infty .5$ \\
\hline sr & 35 & 22 & 18 & 16 & 24 & 21 & 25 & 22 \\
\hline Zn & 77 & 58 & 97 & 92 & 46 & 49 & 45 & 47 \\
\hline $\mathrm{Ca}$ & 19700 & 4520 & 3250 & 4830 & 5130 & 4570 & 9440 & 7530 \\
\hline Ba & 91 & $\pi$ & 82 & 57 & 71 & 65 & $\pi$ & 64 \\
\hline cd & $<0.2$ & $\infty .2$ & $<0.2$ & $<0.2$ & $<0.2$ & $<.2$ & $\infty .2$ & $\infty .2$ \\
\hline $\mathrm{Cr}$ & 12 & 15 & 10 & 9 & 8 & 9 & 7 & 4 \\
\hline $\mathrm{Ag}$ & $<1.0$ & $<1.0$ & $<1.0$ & $<1.0$ & $<1.0$ & $<1.0$ & $<1.0$ & $<1.0$ \\
\hline $\mathrm{Na}$ & 649 & 279 & 132 & 047 & 311 & 287 & 253 & 307 \\
\hline $\mathrm{Ni}$ & 9 & 6 & 9 & 9 & 9 & 9 & 7 & 5 \\
\hline $\mathrm{Cu}$ & $<1.0$ & $<1.0$ & $<1.0$ & $<1.0$ & $<1.0$ & $<1.0$ & $<1.0$ & $<1.0$ \\
\hline$v$ & 47 & 56 & 52 & 59 & 56 & 52 & 58 & 60 \\
\hline sb & $<10$ & $<10$ & $<10$ & $<10$ & $<10$ & $<10$ & $<10$ & $<10$ \\
\hline A! & 1000 & 7510 & 7310 & 5820 & 7710 & 7260 & 6680 & 4970 \\
\hline Mn & 276 & 207 & 309 & 270 & 301 & 287 & 290 & 296 \\
\hline $\mathbf{K}$ & 1590 & 1230 & 1460 & 786 & 1220 & 1200 & 1300 & $6 B 8$ \\
\hline Fe & 26300 & 25300 & 23800 & 23400 & 25400 & 23700 & 26600 & 28000 \\
\hline $\mathrm{Mg}$ & 5150 & 4000 & 4790 & 4980 & 5160 & 4990 & 5020 & 4780 \\
\hline As & 4.0 & 1.2 & 0.95 & 1.2 & 0.9 & 1.3 & 0.9 & $<0.5$ \\
\hline se & $<0.5$ & $<0.5$ & $<0.5$ & $<0.5$ & $<0.5$ & $<0.5$ & 0.5 & $<0.5$ \\
\hline $\mathrm{Pb}$ & 980 & 1140 & 21.4 & 56.4 & 20.8 & 28.4 & 5.5 & 4.1 \\
\hline Nitrate & 1.6 & 3.9 & $<1.0$ & $<1.0$ & 11 & 5.8 & 10.5 & 1.3 \\
\hline Sulfate & 1650 & 1510 & 2.0 & 3.4 & 21.2 & 5.2 & 5.4 & $<1.0$ \\
\hline Fluoride & 2.9 & 3.9 & $<1.0$ & $<1.0$ & $<1.0$ & $<1.0$ & $<1.0$ & $<1.0$ \\
\hline Chloride & 1.9 & $<1.0$ & $<1.0$ & $<1.0$ & 4.3 & 2.1 & 1.2 & $<1.0$ \\
\hline Phosphate & $<2.0$ & $<2.0$ & $<2.0$ & $<2.0$ & $<2.0$ & $<2.0$ & $<2.0$ & $<2.0$ \\
\hline$T 0 x(1)$ & $<1.0$ & $<1.0$ & $<1.0$ & 4.0 & $<1.0$ & $<1.0$ & $<1.0$ & $<1.0$ \\
\hline$\tau \propto c(2)$ & 70.3 & 50.2 & 353 & 461 & 61.5 & 39.3 & 45.9 & 19.0 \\
\hline Ethrgly (3) & $<10$ & $<10$ & $<10$ & $<10$ & $<10$ & $<10$ & $<10$ & $<10$ \\
\hline AR1254(4) & $<1.0$ & 1.3 & $<1.0$ & $<1.0$ & $<1.0$ & $<1.0$ & $<1.0$ & $<1.0$ \\
\hline UNKKOWN ABN & ND & 1.3 & (5) & ND & ND & HD & ND & ND \\
\hline BISPHT (6) & ND & ND & 170 & 17 & ND & ND & ND & ND \\
\hline DIMOPHT (7) & ND & ND & 82 & ND & ND & ND & ND & ND \\
\hline UNK. ALIPH. HC (8) & ND & ND & ND & (9) & ND & ND & ND & ND \\
\hline
\end{tabular}

(1) Total organic helogen

(2) Total organic carbon

(3) Ethylene glycol

(4) Arometic 1254 - PCB

(5) Nine unknow acjd-base-neutrals (ABN's) with estimated cancentrations of 26 to $7900 \mu g / 9$

(6) Bis (2-ethythexyl) phthalate

(7) Di-n-octyl phthalate

(8) Unknown al iphatic hydrocarbon

(9) Nine unknom aliphatic hydrocarbons with estimated concentrations of 22 to $36 \mu g / l$

ND $=$ not detected 
IABLE B.2. Volatile Organic Concentrations in Ground-Water Samples Collected from Seyen Wells in the Vicinity of the 1100 Area on August 3 and $4,1988(a)$

\begin{tabular}{|c|c|c|c|c|c|c|}
\hline Well & $\begin{array}{c}\mathrm{CHCl}_{3} \text { (b) } \\
\mathrm{ppb} \\
\end{array}$ & $\underset{\mathrm{ppb}}{1,1,1-\mathrm{TCA}(\mathrm{c})}$ & $\begin{array}{l}\mathrm{CCl}_{4}(\mathrm{~d}) \\
\mathrm{ppb} \\
\end{array}$ & $\begin{array}{l}\text { TCE }(e) \\
\text { ppb }\end{array}$ & $\begin{array}{c}\mathrm{BDCM}(f) \\
\mathrm{ppb}\end{array}$ & $\begin{array}{c}\mathrm{PCE}(\mathrm{g}) \\
\mathrm{ppb}\end{array}$ \\
\hline $6-S 31-E 13$ & 0.40 & 0.40 & $<0.01$ & 0.15 & $<0.01$ & 0.27 \\
\hline $6-531-E 13$ & 0.40 & 0.39 & $<0.01$ & 0.15 & $<0.01$ & 0.27 \\
\hline $6-S 29-E 12$ & $<0.05$ & 0.06 & $<0.01$ & 0.01 & $<0.01$ & 0.03 \\
\hline $6-S 29-E 12$ & $<0.05$ & 0.06 & $<0.01$ & $<0.01$ & $<0.01$ & 0.03 \\
\hline $6-S 32-E 13 A$ & 0.37 & 0.35 & $<0.01$ & 0.16 & $<0.01$ & 0.26 \\
\hline $6-S 32-E 13 A$ & 0.37 & 0.35 & $<0.01$ & 0.16 & $<0.01$ & 0.26 \\
\hline 6-S32-E13B & 0.50 & 0.39 & $<0.01$ & 0.18 & $<0.01$ & 0.27 \\
\hline $6-531-1$ & 0.38 & 0.04 & $<0.01$ & 0.02 & $<0.01$ & 0.02 \\
\hline $6-\$ 31-1$ & 0.39 & $<0.01$ & $<0.01$ & $<0.01$ & $<0.01$ & 0.01 \\
\hline $6-S 41-13 c(h)$ & 1.11 & 9.35 & $<0.01$ & 0.08 & 0.05 & 0.71 \\
\hline $6-541-13 C$ & 1.13 & 10.15 & $<0.01$ & 0.10 & 0.04 & 0.75 \\
\hline 6-S36-E13A & 0.83 & 2.15 & $<0.01$ & 0.22 & 0.01 & 0.82 \\
\hline $6-S 36-E 13 A$ & 0.81 & 2.19 & $<0.01$ & 0.23 & 0.01 & 0.84 \\
\hline
\end{tabular}

(a) All samples were below detection limits for methylene chloride $<2 \mathrm{ppb}$ and 1,1-dichloroethane $<1 \mathrm{ppb}$

(b) $\mathrm{CHCl}_{3}=\mathrm{chl}$ oroform

(c) $1,1,1-\mathrm{TCA}=1,1,1$-trichloroethane

(d) $\mathrm{CCl}_{4}=$ carbon tetrachloride

(e) TCE $=$ trichloroethylene

(f) $\mathrm{BDCM}=$ bromodichloromethane

(g) $\mathrm{PCE}=$ perchloroethylene

(h) Also known as We11 3000D-1 
TABLE B.3. Analytical Results for Ground-Water Samples from Two Richland Water Supply Wells. Samples were coilected and analyzed by Hanford Environmental Health Foundation. (a) Data are in milligrams per liter unless otherwise noted.

Parameter $\quad \begin{aligned} & \text { Detection } \\ & \text { Limit }\end{aligned} \quad \begin{aligned} & \text { Groundwater } \\ & \text { at 3000D-5 }\end{aligned} \quad \begin{aligned} & \text { Groundwater at } \\ & \text { 3000 Area B (b) }\end{aligned}$

Primary Drinking Water Standards

$\begin{array}{llll}\text { Arsenic } & <0.005 & <0.005 & <0.005 \\ \text { Barium } & <0.1 & <0.1 & <0.1 \\ \text { Cadmium } & <0.0005 & <0.0005 & <0.0005 \\ \text { Chromium } & <0.005 & <0.005 & <0.005 \\ \text { Fluoride } & <0.2 & <0.2 & <0.2 \\ \text { Lead } & <0.005 & <0.005 & <0.005 \\ \text { Mercury } & <0.0005 & <0.0005 & <0.0005 \\ \text { Nitrate } & <0.05 & 0.32 & 0.67 \\ \text { Selenium } & <0.005 & <0.005 & <0.005 \\ \text { Silver } & <0.005 & <0.005 & <0.005 \\ \text { Sodium } & & 2.0 & 2.5\end{array}$

Secondary Drinking Water Standards

Chloride

Copper

Iron

Manganese

Zinc

Sulfate

Total Dis. Solids

Color

$<0.05$

$<0.03$

$<0.01$

$<0.05$

$<1$

$<5$

$<0.004$
Cyanide

VOA $(c)$

Chloroform (ppb)

Bromoform ( $\mathrm{ppb}$ )

p-Chloroto-

luene (ppb)

$\begin{array}{ll}1.3 & 1.0 \\ <0.05 & <0.05 \\ <0.03 & <0.03 \\ <0.01 & <0.01 \\ <0.05 & <0.05 \\ 10.0 & 9.3 \\ 107 & 94 \\ <5 & <5\end{array}$

$<0.004<0.004$
1.73

0.73

0.7

(a) Samples analysed for primary and secondary constituents were collected during August 1987. Samples analysed for volatile organic constituents were collected on November 2, 1987.

(b) Well $3000 \mathrm{~B}$ is one of 11 wells within the North Richland Well Field. The well field and we11 3000D-5 are shown on Figure A.2.

(c) No other VOA were detected. 
TABLE B.4. Analytical Results for Sampled Wells in the 1100 Area - 300 Area Study, 1986 Units in Part per Million (ppm)

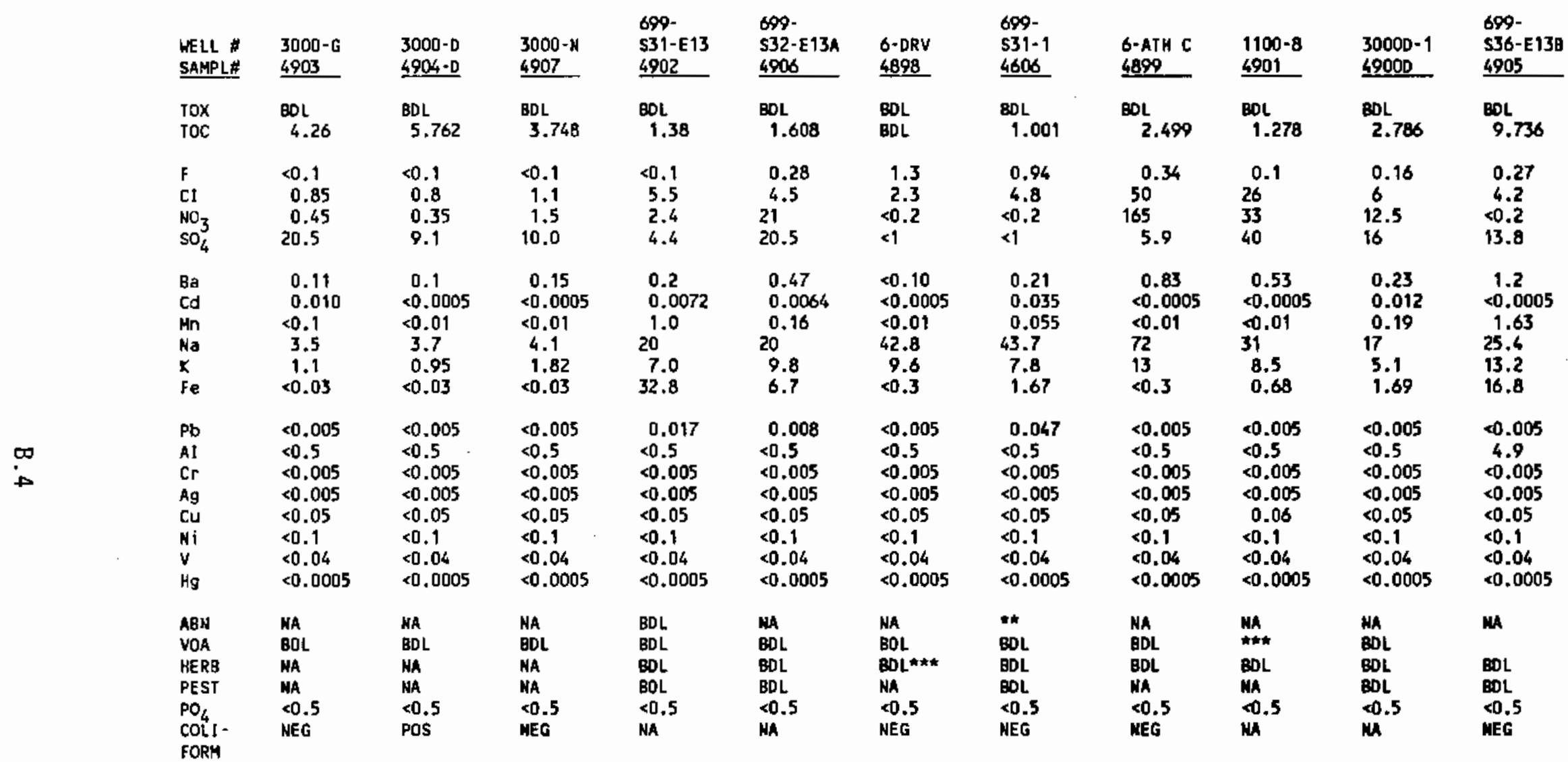

$T O X=$ Total organic hologen, TOC $=$ Total organic carbon, AGN $=$ Acib-bose-neutral (semivolatile) organic compounds

VOA $=$ volatile organic compounds, HERB = Herbicides, PEST $=$ Pesticides, $\mathrm{WA}=$ Not analyzed, BDL $=$ Belou detection $i$ imit.

* Inadequate sample volume for normal detection timit

** bis (2 ethylhexyl) phthalate (code 840 ) 22 ppb (no other ABH's detected)

$\star \star \star$ Methylene Chloride (code A93) $20 \mathrm{ppb}$ (no other vaA's detected)

***\# Inedquate samples (detection limit $=0.0016$ pan) 
TABLE B,5. Results for Water Samples from Richland City Water Supply Wells Analyzed by the State of Washington

Etate of Washinaton

Department of Social and Heaith Services

Division of Health

Public Health Laooratories

1610 N.E. 150th 5t.. Seattle, wa 98155

(206) 36i-2898

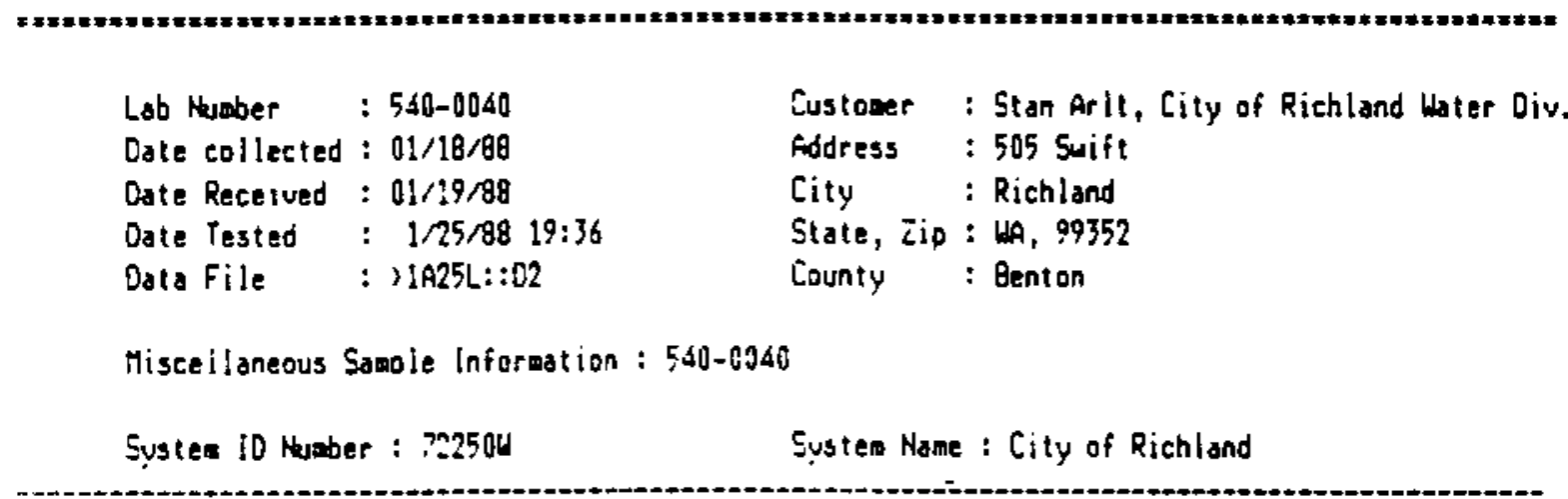

Non-Loaposited Eamolies

OSHS Source Number : $\$ 93$

Source Name : Duke Field

Composited Samoies

Wumer of Sources Used in Comoosite : HA

OSits Source $\$$ 's Composited : H/A

Andysis of Individual Sources Hecessary: It/A

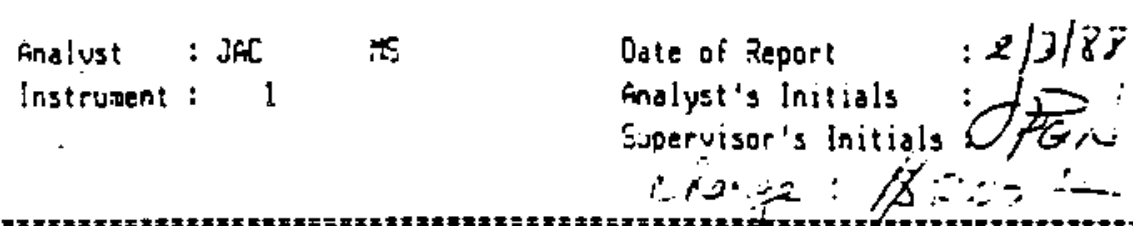

Results of Anatysis by EPA Methad 524

PEGULATED CDMPOUNOS

\begin{tabular}{|c|c|c|c|c|}
\hline $\begin{array}{l}\text { EPA } \\
\text { Code }\end{array}$ & Compound Hame & $\mathrm{MC}(u g / 1)$ & - Amount (ug $/ 1)$ & Compl iance \\
\hline ------ & - & ------ & $-\cdots$ & ------ \\
\hline 2976 & UIMYL CHLORIOE & ? & 0.8 & YES \\
\hline 2977 & 1,1-0ICH_CPOETHMENE & 7 & 0.0 & VES \\
\hline 3781 & 1,1,1-TRICHLOROETHAIE & 200 & 0.0 & YES \\
\hline 2982 & CAREON TETRACHLQRIOE & 5 & 0.0 & YES \\
\hline $2: 90$ & BENZENE & 5 & 0.0 & YES \\
\hline 2980 & 1,2-0ICH OROETHANE & 5 & 0.0 & YES \\
\hline 2984 & TRTCLRORCXTHMEME & 5 & 0.0 & YES \\
\hline 2969 & P-DICHLORCEERZEE & $\pi$ & 0.0 & YES \\
\hline
\end{tabular}

Hote: An Amount of $0.0 \mathrm{ug} / \mathrm{l}$ indicates that the true concentration is less than the detection liait of the exthod.

(page 1 of 3 ) 
Lab Mumber: : $540-0040$

Data File : '!A25L::02

Results of Analusis bv EPA Method 524

(cont inved)

Unregulated Coecounds

Monitor ing Required

\begin{tabular}{|c|c|c|c|}
\hline PA Code & Comoound hase & Prount & $u g /(1)$ \\
\hline 2210 & CHLOROFETHANE & & 0.0 \\
\hline 2214 & EROHOHETHANE & & 0.0 \\
\hline 2216 & CHLOROETHAN & & 0.0 \\
\hline 9964 & METHMERE OHORIOE & & 0.0 \\
\hline 2979 & $:-1,2,-$ DICHEARCETHMEN & & 0.0 \\
\hline 2978 & 1.1-OLCLLGRETHANE & . & 0.0 \\
\hline 2416 & 2,2-DICHLCRDFROPANE & & 3.0 \\
\hline 2380 & C!S-1,2-OICLLCROETMMEIE & & 0.0 \\
\hline 2981 & CHLROFORY & & 1.6 \\
\hline 3410 & 1.1-OICHORLPROPEYE & & 0.0 \\
\hline 2983 & 1,2-0:CHORgPRgPaAE & & 0.0 \\
\hline 2408 & OIRROHOHETHANE & & 0.0 \\
\hline 2943 & BRONLOICHLORQTETHAHE & & 8.0 \\
\hline 2991 & TQLERE & & 0.0 \\
\hline 2985 & 1,1.2-TRICHOROETHAYE & & 0.0 \\
\hline 2987 & TETRACL COPSTTMMEVE & & 0.0 \\
\hline 2412 & 1,3-DIOLLCROPROPAKE & & 0.0 \\
\hline 2944 & CILORCOIEROMDRETHANE & & 0.0 \\
\hline 2989 & CHOOROEERTES: & & 0.0 \\
\hline 2996 & 1,1,1,2-TE-RACHLRCETHANE & & 0.0 \\
\hline $\begin{array}{ll}30 \\
399 ? 2\end{array}$ & ETHM EENZEHE & & 0.3 \\
\hline 2995 & W/P-XYEFIE & & 0.0 \\
\hline 2997 & D-XYLERE & & $: 0$ \\
\hline 2996 & STYFENE & & 0.0 \\
\hline 7942 & BROWDFCM & & 0.9 \\
\hline 2993 & APOHOBENZENE & & 0.0 \\
\hline $24: 4$ & 1., J-TPICH OROPROPANE & & 0.3 \\
\hline 2988 & 1.1,2,2-TETRACHL OROETTAANE & & 0.0 \\
\hline 2965 & Q-OLOROTRLUENE & & 0.0 \\
\hline 2966 & P-GLOROTOLIEAE & & 0.0 \\
\hline 2967 & M-OIOHQRCELSLEGE & $\ldots$ & 0.0 \\
\hline 2968 & D-OICHORDBERIZERE & & 0.0 \\
\hline
\end{tabular}

- Hote: An Arount of 0.0 ug/l indicates that the teue concentration is less than the detection liait of the wethod. 
TABLE B.5. (contd)

Lab thuber : 540-0040

Dato File : JlA25L::02

Results of Analysis by EPA rethod 524

(cont imued)

Unregulated Compounds

Discretianary

\begin{tabular}{|c|c|c|}
\hline EPA Code 1 & Compound Nane & eamount (ug/l) \\
\hline - & H & ------ \\
\hline 2218 & TRIDLLROFLOROTETHANE & 0.0 \\
\hline 2430 & BROHOCHLORDETTHAKE & 0.0 \\
\hline 2994 & ISIPROPYLEENZENE & 0.0 \\
\hline 2998 & N-PROPY EETZESE & 0.0 \\
\hline 2424 & $1,7,5$-TRILETHMLENLENE & 0.0 \\
\hline 2426 & TERT-BUTMEQYZERE & 0.0 \\
\hline 2418 & 1,2,4-TRIMETHYLQELENE & -8.0 \\
\hline 2428 & SEC-EUTY DEHZEYE & 0.0 \\
\hline & P-ISOPROPYLILLEKE & 0.0 \\
\hline 2422 & N-AUTYSERZENE & 0.0 \\
\hline 2378 & I,2,0-TRICHLORCSENZENE & 0.0 \\
\hline 2248 & HAFTHALEFE & 0.0 \\
\hline 2246 & HEXACHEROQDTAOIENE & 0.0 \\
\hline 2420 & 1,2,3-TRICHLROBENENE & 0.0 \\
\hline
\end{tabular}

- Hote: An Amount of 0.0 yo/l indicates that the trueconcentration is less than the detection livit of the enthod.

(poge 3 of 3 )

B.7 
State of thastingaton

Deportment of Social and Health Services

Division of Health

Public Health Laooratories

1610 K.E. 150th St., Seattle, Wa 98155

(206) $361-2898$

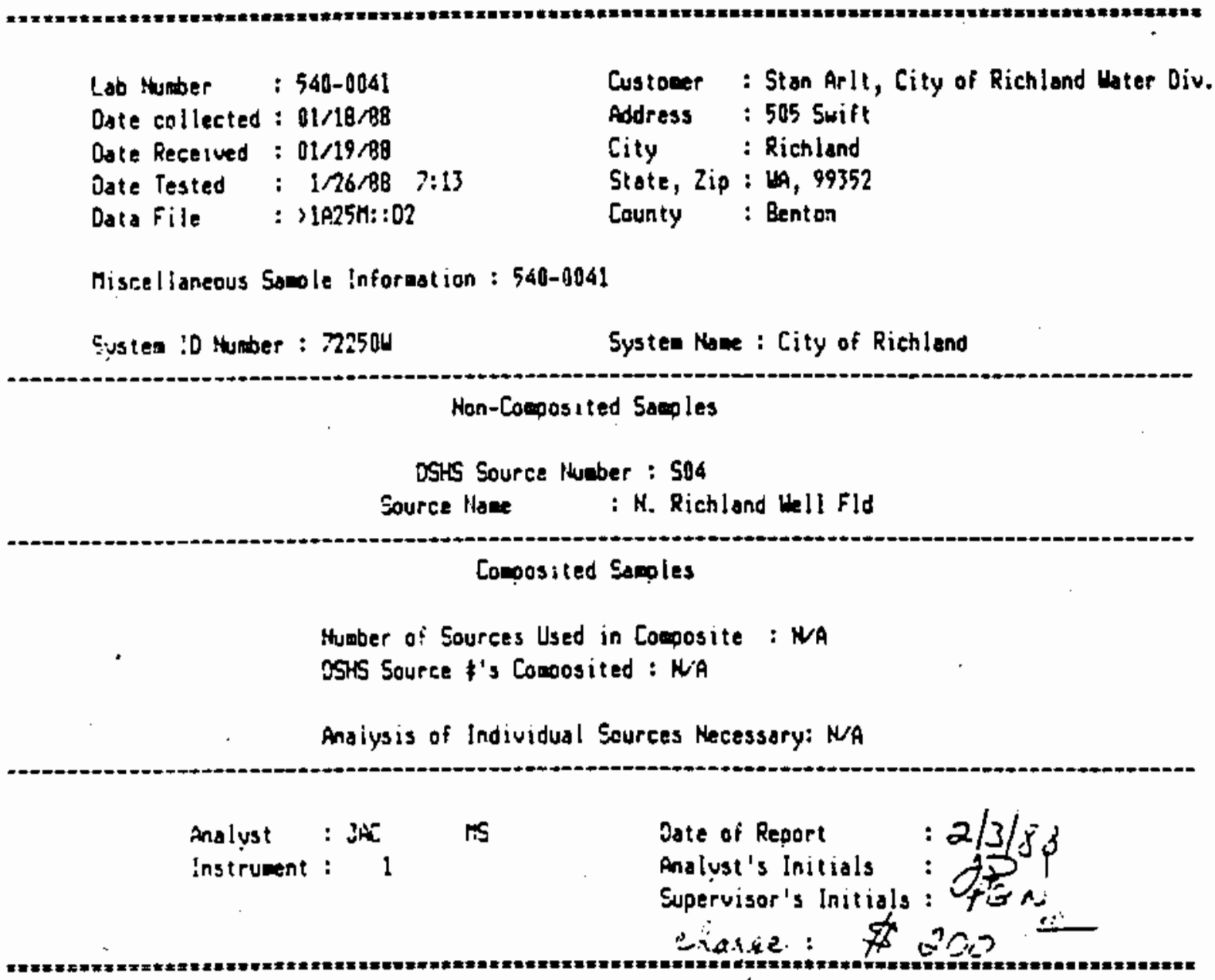

Results of Analysis by EPA ine thod 524

EPA

Cade :

Conoound Nace

REQLATEO COMPOADS

-.-nen

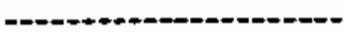

\begin{tabular}{|c|c|}
\hline Cade $\leq$ & Compound Nase \\
\hline 2976 & UIMM CHLRIOE \\
\hline 7 & 1.1-DICHOROETMLEE \\
\hline 2981 & 1,1,1-TRIOLCONETHAYE \\
\hline 82 & CARBOA TETRACHCRIOE \\
\hline 2990 & EERTENE \\
\hline 990 & 1.2-OICHLCROETHANE \\
\hline 984 & TRICHLORDETIMEE \\
\hline 69 & P-olat ordeganete \\
\hline
\end{tabular}

- itste: An munt of $0.0 \mathrm{ug} / 1$ indicates that the true concentration is less thon the detection liail of the rethod.

(page 1 of 3$)$

B.8 
Lab thuber: 5 a AB-00S:

Data File : :1A25M::02

Results of Analysis b.y EPA Methad 524

(cont inued)

Unregulated Cemoounds

Monitoring Required

\begin{tabular}{|c|c|c|c|}
\hline EFA Code & Cionoound Nase & Amount & $(\operatorname{ug} / 1)$ \\
\hline 2210 & CHOROUETHANE & & 0.0 \\
\hline 2214 & BROHANETHANE & & 0.0 \\
\hline 2916 & CHAOPCTHANE & & 0.0 \\
\hline 2364 & RETHMESE CHLDRIOE & & 0.0 \\
\hline 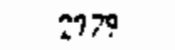 & $T-1,2,-9, C H$ OROETHMENE & & 0.0 \\
\hline 4978 & 1.1-OILH GRLETHAKE & - & 0.0 \\
\hline 2616 & 2,2-OICH OPOFROPAYE & & 0.0 \\
\hline 2380 & CIS-1,2-DICHLOROEIHYLEE & & 0.0 \\
\hline 2941 & CHLOROFCRM & & 13.6 \\
\hline 2430 & 1,1-OICHEOROPPOPENE & & 0.0 \\
\hline 2983 & 1,2-DIOLCROPROPANE & & 0.0 \\
\hline 2408 & DIBRDTRLTHANE & & 0.0 \\
\hline 2943 & BRDNOC ICHLCPOHETHANE & & 1.5 \\
\hline 2991 & TOL'ERE & & 0.0 \\
\hline 2985 & $1,1, \hat{2}-$ TRICHLOROETHANE & & 0.0 \\
\hline 2987 & TETRACHQRROETHMLNE & & 0.0 \\
\hline $24: 2$ & 1,3-OICHOROPROPANE & & 0.0 \\
\hline 2944 & CLLCP.JOIEPOMTOAETHAHE & & 0.0 \\
\hline 2989 & CHeGRDRENLE:EE & & 0.0 \\
\hline 3996 & $1,1, \therefore, 2$-TETRACHLLOROETHANE & & 0.0 \\
\hline$: \div 92$ & ETHM GENZENE & & 0.0 \\
\hline 2905 & M/P-XTLEe & & 0.0 \\
\hline 2997 & I-XYLEIE & & 0.0 \\
\hline 2996 & STMEEE & & 0.0 \\
\hline $294 ?$ & SROMSFORI & & 0.0 \\
\hline 2092 & BROHOPSWZEE & & 0.0 \\
\hline $2 \leq 14$ & $1,2,3$-TR!CHLCRCPROPANE & & 0.8 \\
\hline $2: 88$ & 1,1,2,2-TETRACTL OROETHSHE & & 0.0 \\
\hline 2955 & D-CHeCROTLLUERE & & 3.0 \\
\hline 2966 & P-OHLOROTQLUENE & & 0.0 \\
\hline 2967 & M-OICHLOROEENRSNE & - & 0.0 \\
\hline 2968 & O-DICH OROBBETEENE & & 0.0 \\
\hline
\end{tabular}

* Hate: An Amount of 0.0 uo $/ !$ indicates that the true concentration is less than the detection linit of the method. 
IABLE B.5. (contd)

Lab Nusber : 540-004!

; Datafile : :IA25M::C?

Resules of Analys:s by EPA Rethod 5:4

(cont inued)

Uhrequlated Comounds

Discretianary

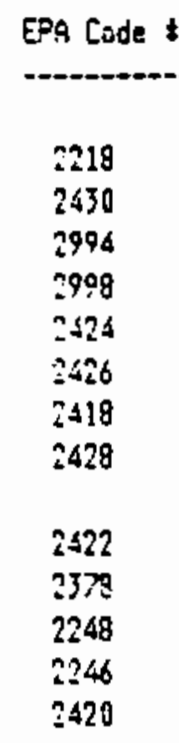

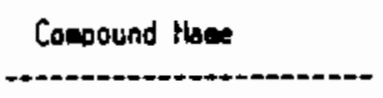

afount (ug/)

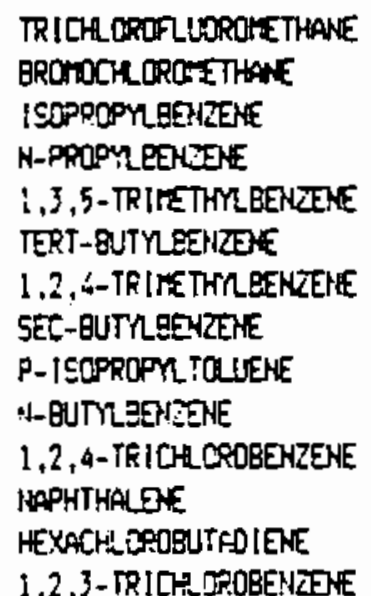

0.8

0. 1

0.0

0.0

ง. 0

0. 0

$-0.0$

9.0

0.0

0.0

0.0

0.0

9.0

0.0

\footnotetext{
+ Hote: in finount of 0.8 ugit indicates that the trueconcentration 15 less than the detection Iarit of the eethod.
}

(osce 3 of 3$)$ 


\section{APPENDIX C}

\section{FUNCTIONAL DESIGN AND CONSTRUCTION STANDARDS FOR}

GROUND-WATER MONIIORING WELLS 


\section{APPENDIX C \\ FUNCTIONAL DESIGN AND CONSTRUCTION STANDARDS FOR \\ GROUND-WATER MONITORING WELLS}

\section{INTRODUCTION}

Five ground-water monitoring wells will be drilled between the 1100 Area and the Richland water supply wells. The purpose of this appendix is to discuss the minimum design, and construction, standards required to meet the objectives of environmental monitoring. Three tasks are discussed:

- Task 1. Drilling

- Task 2. Geologic sampling

- Task 3. Well construction

\section{LOCATION AND DEPTH}

Welis will be installed at five locations near or adjacent to the 1100 Area. Well locations are considered tentative until underground and overhead utilities are checked by Westinghouse personnel before drilling. Estimated locations are shown in Figure A.2. Approximate depths are listed in Table 3.2.

\section{HYDROGEOLOGY}

The subsurface stratigraphy consists of unconsolidated and consolidated deposits of clay, silt, sand, gravel, cobbles, and boulders deposited in fluvial and glaciofluvial environments. The deposits may be locally cemented.

The depth to water in the area is approximately $50 \mathrm{ft}$ below ground surface. The direction of ground-water flow is thought to be toward the east. 
TASK 1 DRILLING

Method

A cable-tool drill rig is the recommended drilling method (Kasper and Myers 1987). Temporary steel casing will be driven concurrent with drilling because most of the geologic formations are expected to be uncemented and unconsolidated. Hard tool may be used for drilling through gravel, boulders and cobbles, otherwise core barrel drilling is preferred. A split-tube sampler may be used to take relatively undisturbed samples of specific layers when necessary.

Precaution should be taken to prevent contamination of the well with oil and grease.

Borehole Size

The minimum borehole diameter will be 8-in. (Kasper and Myers, 1987). Telescoping of successively smaller casings will be required in each well to achieve a minimum 8-in. diameter borehole, prevent hydraulic interconnection through any confining layers, and prevent problems in pulling the casing.

If perched water is encountered, special precautions will be taken to prevent contamination migration as follows. In no instance shall any one size of temporary casing be extended across the entire thickness of a confining layer, since this may connect two aquifers or a perched zone of water to the ground water. The uppermost casing shall not penetrate less than $2 \mathrm{ft}$ nor more than $5 \mathrm{ft}$, or $20 \%$ of the known thickness of the confining unit. The smaller casing shall be telescoped through the remainder of the confining layer into the next permeable unit. Bentonite slurry will be added to the annulus while drilling through the confining layer to form a seal.

Qualification of Drillers

Drillers should have at least 5 years experience as a driller and be licensed in the state of Washington. Drillers should have a minimum of 2 years experience specificaliy in cable-tool drilling. At least 1 year of this experience should be as a driller, not as a helper or a derrick man. The cable-tool drillers should have specific experience for underreaming and pulling casing. In addition, the drillers should have extensive experience 
installing monitoring wells for hazardous waste investigations. In accordance with Occupational Safety and Health Act (OSHA) regulations (29 CFT Part 1910.120), the drilling company that is contracted must meet OSHA medical, monitoring, and hazardous waste training requirements.

\section{Equipment}

The drilling contractor will provide all equipment required complete all tasks outlined in the detailed drilling specifications. Equipment will be in good working order and steam cleaned just before arriving on site. Equipment failure is the responsibility of the contractor, and any time lost because of equipment failure will constitute downtime.

\section{Temporary Materia]s}

All temporary casing should be clean, undamaged sections of carbon steel pipe. The following sizes may be required: 16-, 12-, 10, and 8-in. Casing should be Schedule 40 and meet ASTM specification A53 Grade B or API $5 L$ Grade B (ASTM 1986).

Welding

Because retrieval of temporary casing may be difficult, precautions will be taken to help maintain high-integrity welds (i.e., beveling cuts before welding, grinding, and crushing to remove slag between passes). Welds should not be ground flush to the casing. An extra bead should be considered on welds near the top of the casing string. The contractor will be responsible for broken welds.

Drill Cyttings and Water Disposal

Cuttings and drill-water bailed or cored from the hole will be surveyed with radiation and organic vapor detection monitoring instruments. Materials with any contamination above prescribed action levels will be stored on site until the proper disposal method is identified. Uncontaminated soil and water will be disposed of in the vicinity of the borehole. 


\section{TASK 2 GEOLOGIC SAMPLING}

The drilling contractor will be responsible for proper operation of the equipment to be used both for the sediment sample collection and for the aquifer tests. Sediment samples will be collected at a minimum of $5 \mathrm{ft}$ intervals during installation of the well. Samples will be collected using a core barrel where possible, or with a bailer when using the hard tool method. Samples collected for chemical analysis should be collected with the core barrel method, or split-spoon.

\section{TASK 3 WELL CONSTRUCTION}

Generic diagrams of the proposed shallow and deep well constructions are presented in Figures A.8 and A.9. The wells shall be constructed such that they meet or exceed the requirements of WAC 173-160 and 162 .

\section{CONSTRUCTION MATERIALS}

\section{Stainless Steel Screens and Casing}

The wells should be constructed of 4-in. diameter, flush-threaded stainless steel well screen and pipe (Kasper and Myers 1987). The monitoring well casing will be Schedule 5S, meeting ASTM specification A312 or A778 (ASTM 1986). The end fittings will be Schedule 40, double entry Stub, ACME flush screw threads with a Viton 0 -Ring on male end fittings. The pipe should be furnished in various lengths to eliminate the need for cutting. The length and slot size of the well screens will be selected by the site geologist. All well screens will be the continuous wire wrap type. A bottom plate should be welded to the bottom of each well screen. Silt traps should not be used. A11 factory welding of fittings to screen or casing and welding to the lock in caps be performed with a MIG or IIG stainless steel wire feed welding process. The stainless steel wire feed must be of the same material as the casing or screen being welded. The stainless steel well screen and pipe may be composed of these four types: 304, 304L, 316, and 316L (Kasper and Myers 1987). Type 304 and 304L or 316 or $316 \mathrm{~L}$ may be combined. However, individual sections should not be composed of 304 or $304 \mathrm{~L}$ and 316 or $316 \mathrm{~L}$ welded together. 
To minimize damage to the well screen and pipe, the following should be required:

- All male threads should be protected from damage.

- All pipe and screen must be wrapped in polyethylene packaging.

- Each box containing pipe and screen should be clearly marked as to the material type.

- The outside of the shipping box shall be clearly labeled with the description (including material type), quantity, and part number of the stainless steel product contained in the shipping box.

- Stainless steel caps and centralizers must be the same type (not just the same series) of stainless steel as the well casing to which it is attached.

In areas where constituents of concern include chromium or certain radionuclides (particularly selenium-75, strontium-85 and -90 , and cesium-137) the corrosion-resistant stainless steel should be passivated by the manufacturer or supplier to prevent the release of chromium from the well screen and pipe or adsorption of constituents (Raber et al. 1983). Passivation is also needed for cathodic protection in high chloride or fluoride environments (e.g., areas where large amounts of $\mathrm{HCL}$ have been released into the ground water). Pretreatment used before the passivation shall be in accordance with $\mathrm{Mi}$-spec-5002, shall leave the surface of the pipe and screen free of oil, grease, rust, scale, and other foreign matter, and shall have no deleterious effect on the material properties. The stainless steel parts shall be passivated in accordance with Mi1-spec QQ-P-35B. Specifically, these parts shall be immersed in a aqueous solution of 38 to $42 \%$ nitric acid, by volume, in accordance with $0-\mathrm{N}-350$, and 2 to $3 \%$ sodium dichromate, by weight, in accordance with $0-S-595$. The temperature of the solution should be 70 to $120 \mathrm{~F}$ and the materials should be immersed for 25 to $30 \mathrm{~min}$; this is followed by a clean rinse and an immersion in a 4 to $6 \%$ sodium dichromate solution for 30 min followed by a final clean-water rinse and an air drying period. 
The stainless steel casing, well screen, and accessories (caps, centralizers, etc.) must conform to all applicable items in the above specifications with all supporting test data certified and submitted to and approved by PNL. and $\mathrm{XEH}$, before shipment.

\section{Filter Pack}

Because the rate at which sand grains will settle in water is controlled by particle size and shape, it is desirable to obtain a filter pack that consists of sand grains that are uniform in shape and size. The filter pack surrounding the well screen should consist of kiln-dried, rounded and spherical grains of sand composed of at least $95 \%$ quartz. The sand grains shall be composed of subrounded to rounded grains; that is, at 1east $90 \%$ of the grains shall have a Powers roundness of 4.0 to 6.0 , or a Krumbein roundness of 0.6 to 1.0. The Maximum Projection Sphericity (Sneed and Folk 1958) shall be 0.6 to 1.0 . The sand particles should be very uniform in size. Specifically, the uniformity coefficient should be equal to or less than 2.5. The effective size shall be proportional to the sieve size. For example, the 10-20 U.S. mesh size shall have an effective size of $0.033-$ to $0.045-i n$. (1.0 to $1.2 \mathrm{~mm}$ ), and the 8-12 U.S. mesh size shall have an effective size of $0.067-$ to $0.079-i n$. (1.7 to $2.0 \mathrm{~mm})$. The paper sacks containing the sand should have polyethylene liners to prevent contamination and water damage. The two currently approved supply sources for the quartz sand pack are the Fountain Sand and Gravel Company, Pueblo, Colorado, and the Colorado Silica Sand, Inc., Colorado Springs, Colorado.

\section{Secondary Filter Pack (Optional)}

A secondary filter is often desirable and is mandatory if a bentonite slurry is to be placed above the sand pack rather than a seal of pellets, crumbles, or granules. A secondary filter is a 1 to $2 \mathrm{ft}$ layer of material that is placed in the annular space between the filter pack and the bentonite seal or between the bentonite seal and the grout. Unlike the filter pack, which is very uniform in size, the secondary filter above the sand pack should be less uniform. The greater variation in size will allow the coarser grained sand fraction of the secondary filter to settle first on equivalently coarse sand pack and second on regressively finer-grained sand. Thus, the 
finest sand particles are adjacent to the overlying finer-grained bentonite slurry or crumbles. This prevents migration of the bentonite into the filter pack. A properly graded secondary filter shall consist of two particle sizes of sand including: 20 to 40 mesh (effective particle size of 0.016 - to 0.019-in. (0.4 to $0.5 \mathrm{~mm})$ and 40 to 140 mesh, commonly called 100 mesh (effective particle size of $0.0055-$ to $0.0066-i n$. $(0.14$ to $0.17 \mathrm{~mm})$. The sand materials shall be packaged in sacks with polyethylene liners to prevent contamination and water damage.

\section{Bentonite Sealants}

Bentonite pellet seals shall be composed of commercially available pellets that have a dry bulk density of at least $80 \mathrm{lb} / \mathrm{ft}^{3}$ and are a nominal 0.25 - to $0.375-i n$. in diameter.

Granular bentonite shall be composed of coarse, granular bentonite crumbles, 8-20 U.S. sieve size. The sacks of bentonite shall be shipped on pallets and sealed with plastic sheeting.

The bentonite grout shall be a bentonite clay grout powder with a specific gravity of 2.5, a dry bulk density of $55 \mathrm{lb} / \mathrm{ft}^{3}$ and a $\mathrm{pH}$ of 9 to 10.5 . The bentonite grout powder shall be Volclay Grout (or an approved equal) below the water table and regular bentonite powder and/or granules above the water table. The sacks of bentonite shall be shipped on pallets and sealed with plastic sheeting. Water from an approved source shall be mixed with these powders or granules to form thick, bentonite slurries. When used, slurry seals shall have a thick, batter-like consistency with a Marsh Funnel viscosity of at least $100 \mathrm{sec}$. Volclay grout should maintain a weight of 94.3 to $94.5 \mathrm{jbs} / \mathrm{ft}^{3}$. Maximum placement thickness of a slurry seal in a single lift should be $25 \mathrm{ft}$.

\section{Cement Grout}

Cement grout should consist of a mixture of Port] and cement (ASTM C150; ASTM 1986) and water in the proportion of 5 to $6 \mathrm{gal}$ of clean water per bag (94 1bs or $1 \mathrm{ft}^{3}$ ) of cement. Because the volumetric shrinkage is about $17 \%$, an additive should be added to the cement to cause it to expand on setting, thus providing a tighter seal (Aherns 1970). The two primary choices are 
aluminum powder ( $1 \%$ ) and gypsum ( 3 to $6 \%$ ). Both additives also accelerate setting, but aluminum powder produces a stronger grout and is less expensive than gypsum.

\section{Concrete Pad and Seal}

Concrete, when used in monitoring well construction, shall be composed of either premixed, bagged concrete, or a mix of six sacks of cement per cubic yard. Except for an air entraining agent, neither additives nor borehole cuttings shall be mixed with the concrete. With the addition of water, the maximum slump should be $6-i n$.

\section{Guard Posts}

Protective steel posts should be composed of carbon steel casing that is new, free from pits or breaks, and has a 4-in. nominal diameter. The pipe shall be Schedule 40 meeting ASTM specification Al20 (ASTM 1986). The individual 6-ft segments shall have straight, beveled cuts. The posts should be painted yellow to make them easily visible.

\section{INSTALLATION PROCEDURES}

\section{Stainless Steel Casing and Screen}

The stainiess steel well screen and casing is lowered to the desired depth and suspended in position until the sand pack has been emplaced. Centralizers should be attached immediately above the well screen and at the appropriate distances along the length of the casing necessary to assure an adequate seal.

\section{Filter Pack}

The volume of the filter pack required to fill the annular space between the well screen and the well hole must be computed and carefully measured. The filter pack should be extended at least $1 \mathrm{ft}$ above the top of the screen for every $3 \mathrm{ft}$ of screen length, up to a maximum of $5 \mathrm{ft}$ above the top of the screen. While suspending the riser pipe, the temporary casing or should be carefully withdrawn until the lowermost point on the casing is exactly at the top of the filter packed portion of the hole; this may be accomplished in increments, if necessary. 
Secondary Filter Pack

To prevent downward migration of a bentonite slurry into the sand pack, a carefully measured volume of secondary filter sand should be placed above the top of the primary filter pack. This filter material is poured into the annular space through a clean, flush threaded, tremie pipe that has been lowered to no more than $20 \mathrm{ft}$ above the placement interval. If used, the secondary filter should be 1 to $2 \mathrm{ft}$ thick. Where conditions warrant, the secondary filter may be eliminated.

Bentonite Seal

Where a bentonite seal is required above the water table and the pellet seal, bentonite granules or crumbles shall be used to seal the annular space to within $21 \mathrm{ft}$ below land surface. Pellets or crumbles should be dropped into the annulus slowly and measured frequently to ensure that the bentonite particles are not bridging. The use of Volclay grout will be limited exclusively to placing the grout through a tremie in annular spaces below the water table. Bentonite slurry shall be pumped under pressure into the annulus. The opening of the discharge pipe should be immediately above the filter pack or point of placement. The end of the tremie pipe must be equipped with a side discharge to prevent displacement of the filter pack and seal materials. A minimum of $2 \mathrm{ft}$ of sealant material (sand, pellets, grout slurries, et.) will be maintained in the annulus during placement. It is recommended that no more than $5 \mathrm{ft}$ of sand pack, $2 \mathrm{ft}$ of bentonite pellets or crumbles, or $20 \mathrm{ft}$ of bentonite or grout be installed before the casing is pulled back. The grout shall be a mixture of volclay bentonite-based grout (dry mix) and water and shall have a mud balance weight between 9.3 to 9.5 lbs per gal.

\section{Cement Grout Surface Seal}

The cement grout slurry with $1 \%$ aluminum powder will be added into the open annulus from $21 \mathrm{ft}$ below ground surface to within $3 \mathrm{ft}$ of land surface. If the grout settles, additional grout will have to be added to refill the annulus to within $3 \mathrm{ft}$ of the ground surface. 


\section{Concrete}

The concrete mix for the surface well seal and pad shall be superior in strength and resistance to freezing and thawing. The concrete surface structure shall be placed within the annular space from a depth of $3 \mathrm{ft}$ and extend to the ground surface. This same concrete mix shail be used to construct a 4-ft by 4-ft by $6-\mathrm{in}$. concrete pad around and centered on the well. A brass marker shall be placed in the concrete before it sets. Numbers should not be stamped into the brass marker for at least 7 days after the concrete has been placed.

\section{Guard Posts}

Four steel guard posts shall be placed at the corners of the pad. Three of the posts shall be set in concrete to $2.5 \mathrm{ft}$ in depth. The fourth post should be set in a sleeve to allow easy removal of the post for equipment access to the well.

Pump

A Hydrostar pump shall be placed in each well to facilitate groundwater sample collection.

Hell Cap

A lockable well cap shall be placed on the well and locked to prevent unauthorized access to the well.

\section{Quality Assurance}

The reliability of ground-water samples analyzed for hazardous constituents are known to be affected by drilling methods and equipment used. The following quality assurance procedures will be followed:

1. All equipment in contact with earthen materials or borehole fluids shall be steam-cleaned (high pressure at $80 \mathrm{psi}$ and high temperature at $180 \mathrm{~F}$ is also acceptable) between each borehole. The contractor shall supply the equipment necessary for this decontamination. Tools and sampling equipment (e.g., split-spoon sampler, submersible pumps) to be used for soil sampling or aquifer testing 
shall be decontaminated by wiping or scrubbing off visible particulate matter; washing with a laboratory-grade detergent (e.g., trisodium phosphate), clean water and methanol (technical grade or better) rinsing; and final rinsing with clean water free of oils and contaminants of concern. The trisodium phosphate, methanol (where necessary), distilled water, and all other items (e.g., brushes, clean water, etc.) must be provided and obtained by the contractor.

2. The soil around the borehole is a potential contamination source; therefore, all materials (casings, drill rods, etc.) will be stored on stands, pallets, support vehicles, etc.

3. Only preapproved lubricants, such as "Green Stuff," will be allowed on any drilling tools. No petroleum-based lubricants will be used.

4. Any water used during drilling will be obtained from a designated source. No chemical additives (i.e., drill foam) will be used.

5. All monitoring well pipe, screen, and accessories (cap, centralizers) shal1 be cleaned and decontaminated at the factory.

6. Proper installation of each well is critical to the integrity of the data collected from it. Design specifications will be followed. Any deviations from prescribed design must meet the approval of the WHC cognizant engineer or his designee (i.e., site geologist).

Proper disposal of discharge water during well development and sampling is often a problem at hazardous waste sites. An Effluent Monitoring Plan will be in place before pumping begins. This will identify the acceptable limits of contamination for disposal of pumped water to the ground surface. A ground-water sample will be collected and analyzed before development. If no contamination is detected above acceptable limits, the development will proceed, with discharge to the ground surface at least $1000 \mathrm{ft}$ from any waste disposal facilities. If ground-water contamination is detected, development will not occur until a decision can be made on whether treatment and disposal of pumped water is necessary. 


\section{REFERENCES}

Ahrens, T. P. 1970. "Basic Considerations of Well Design: Part III." Water Well Journal 24(3)47-51.

Folk, R. L. 1968. Petrology of Sedimentary Rocks. Hemphill's, University of Texas, Austin, Texas, $170 \mathrm{p}$.

Kasper, R. B., and D. A. Meyers. 1987. Engineering Study: Technical

Evaluation of Materials and Methods for the Construction of Ground-Water Monitoring Wells at Resource Conservation and Recovery Act Requlated LowLevel Burial Grounds in the 200 Areas, Hanford Site. SD-RE-ES-037, Rockwell Hanford Operations, Richland, Washington.

Raber, E., J. Garrison and V. Oversby. 1983. "The Sorption of Selected Radionuclides on Various Metal and Polymeric Materia.s" Radioactive Waste Management and the Nuclear Fuel Cycle 4(1)41-52.

Sneed, E. D. and R. L. Folk. 1958. "Pebbles in the Lower Colorado River, Texas, A Study in Particle Morphogenesis." Journal of Geology 66:139-183. 


\section{APPENDIX D}

\section{DATA COLLECTION AND DOCUMENTATION PROCEDURES}

FOR MONITORING WELL INSTALLATION 


\section{APPENDIX D}

\section{DATA COLLECTION AND DOCUMENTATION PROCEDURES FOR \\ MONITORING WELL INSTALLATION}

This appendix lists the procedures to be used during drilling and well construction activities by Pacific Northwest Laboratory in support of the well installation and sampling of 1100 Area environmental monitoring wells. 
Procedure for Collection and Documentation of Orflling Data, P-6 for RCRA Compliance/Hazartous Materials Monitoring

\subsection{OBJECTIVE}

This procedure provides standardized methods for the collection and documentation of sediment samples and well construction data, for wells drilled by the cable tool method.

\subsection{APPLICABILITY}

This procedure applies to work performed by well site geologists curing well construction.

\subsection{RESPONSIBILITIES}

\subsection{Well Site Geologist}

- Collect and document drilling data

\subsection{Senior Technical Reviewer}

- Review and sign/date Well Completion Report/Title III Inspection List

- Review and sign/date As-Euilt oiagram

- Reytew and sign/date Orill Logs

\subsection{PROCEDURE}

\subsection{Collection of Sediment Sanoles}

Sediment samples shall be collected at 5-foot interials and changes in formation by the well stte geologist. All drill cuttings to be samplei shall be collected from the drtller in a 5 gallon bucket.

Two pint jars shall then be fflled from the bucket. One jar shall be submitted for laboratory analysis and the other retained for archiving.

A label tndfeating well number, depth, date, drilling method and initials of the geologtst collecting the sampie shall be placed on eaci sampie jar. Well number, depth and date shall also be written on the lid of each Jar.

\begin{tabular}{|c|c|c|c|}
\hline $\begin{array}{l}\text { Approvals: } \\
\text { Project Mana }\end{array}$ & & ep. & 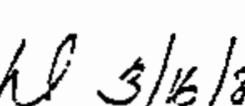 \\
\hline $\begin{array}{l}\text { Procedure No: } \\
\text { P-6 }\end{array}$ & $\underset{0}{\text { Revision }}$ & $\begin{array}{r}\text { Oate issued: } \\
3-16-87\end{array}$ & Page 1 of 3 \\
\hline
\end{tabular}


Procedure for Collection and Oocimentation of Orilling Data, P-6 (continued)

If the well is being drilled with a drive barrel above the water table, a moisture sample shall also be collected from the bucket. Each moistirs: sample shall be sealed with white identification tape. The well number, depth, date and initials of the geologist collecting the sample shall be recorded on the identification tape with indelibie ink. Each moisture sample shall then be doubly wrapped in a plastic bag, and taped shut.

The remainder of the sample in the bucket shall be used for sedinent sample description.

\subsection{Documentation of Sediment Samoles and Well Construction Oata}

Sedinent sample descriptions and well construction data for each well shall be recorded on a Well Completion Report/Title III Inspection List (Attachment 1), As-Buflt Diagram (Attachment 2) and Orili Log (Attachrient 3). These data shall be recorded dafly by the well site geologist. Nonapplicable 1 tems shall be designated $N / A$.

The Well Completion Report/Title III Inspection List provides a compiete sumutary of weli construction and completion data. Data recorded on the Well Completion Report/Title III Inspection List shail include: generai project and well inforation, drilling method, completion data, casting data, perforations, screen, annular seal, geophysical logging, aquifer testing and other applicable ttens. Casing data, periorations, screan, annular seal, geophysical logging, aquifer testing and other applicable items shall be approved by the well site geologist. After coiriletion of the well, an overall reyiew of the Well Completion Report/Title III Inspection List shall be periormed by the Sentor Techntcal Reviewer.

The As-built diagram is a graphical representation of the well construction, geologic and hydrologic data. Data recorded on the As-Built Otagram shall include: well number, geologist, page number, construction data, depth in feet, geologic and hydrologic data. After completion of the wel1, an overali review of the As-aulit ojagram shall be performed by the Senior Technical Reviewer.

The Drill Log contains detailed descriptions of the sediment santies and well construction data. Data recorded on the orill Log shall incluce: geologist name, date, rig, well number, depth at st3rt, depth at finish, computer number, project number, subcontract number, total casing, depth, dritl method, wet/dry sample, It thologic description including moisture sample data, time, drilling comments and remarks. A new Drili Log shall be used eech day. After compietion of the day's activities, the well sita geologist shall sign and date the Orll Log. After completion of the well, an overall review of the Orill Logs shall be perfomed by the Sentor Technical Reviewer.

\begin{tabular}{|l|c|c|c|}
\hline $\begin{array}{c}\text { Procedure No. } \\
P-5\end{array}$ & $\begin{array}{c}\text { Revision to. } \\
0\end{array}$ & Pagse 2 of 3 \\
$3-16-57$ &
\end{tabular}


Procedure for Collection and Documentation of Orllling Data, P-6 (continued)

\subsection{Descriotion of Sediment Samoles and Well Construction' Data}

Detailed descriptions of the sediment samples and well construction data shall be recorded on the Orill Log under "Lithologic Description" by the well site geologist.

Sediment sample descriptions shall include the following information as a minimur: lithologic name, texture, sorting, gross mineralcgy of the framework and matrix, roundness of the framework and matrix, wet/dry color, reaction in hydrochloric acid (HCl), consolidation and changes in li thology.

Well construction data sinall include the following fnroration as appitcable: drill method, drilil depth, conpletion depth, drill rata, casing (type, size, depth and lengths), perforations (type, depth and schedule), screen (type, length, slot size and depth), annular seal (type, interval and volume), packer (type, size and depth), well development and depth to water.

\subsection{Data Manaqement}

After conpletion of the project, the original Well Completion Report/Titie III Inspection List, As-Built Olagram and Orill Logs for each well shall be retained by $v$. L. McGhan of the PNL Geoselences Departanent. A copy of each completed form shall be retained by the PML Recordis Retention Center.

\begin{tabular}{|c|c|c|c|}
\hline $\begin{array}{c}\text { Procedure Ho. } \\
\text { P-6 }\end{array}$ & $\begin{array}{c}\text { Revision Ho. } \\
0\end{array}$ & $\begin{array}{c}\text { Oate Issied: } \\
3-16-87\end{array}$ & Page 3 of 3 \\
\hline
\end{tabular}




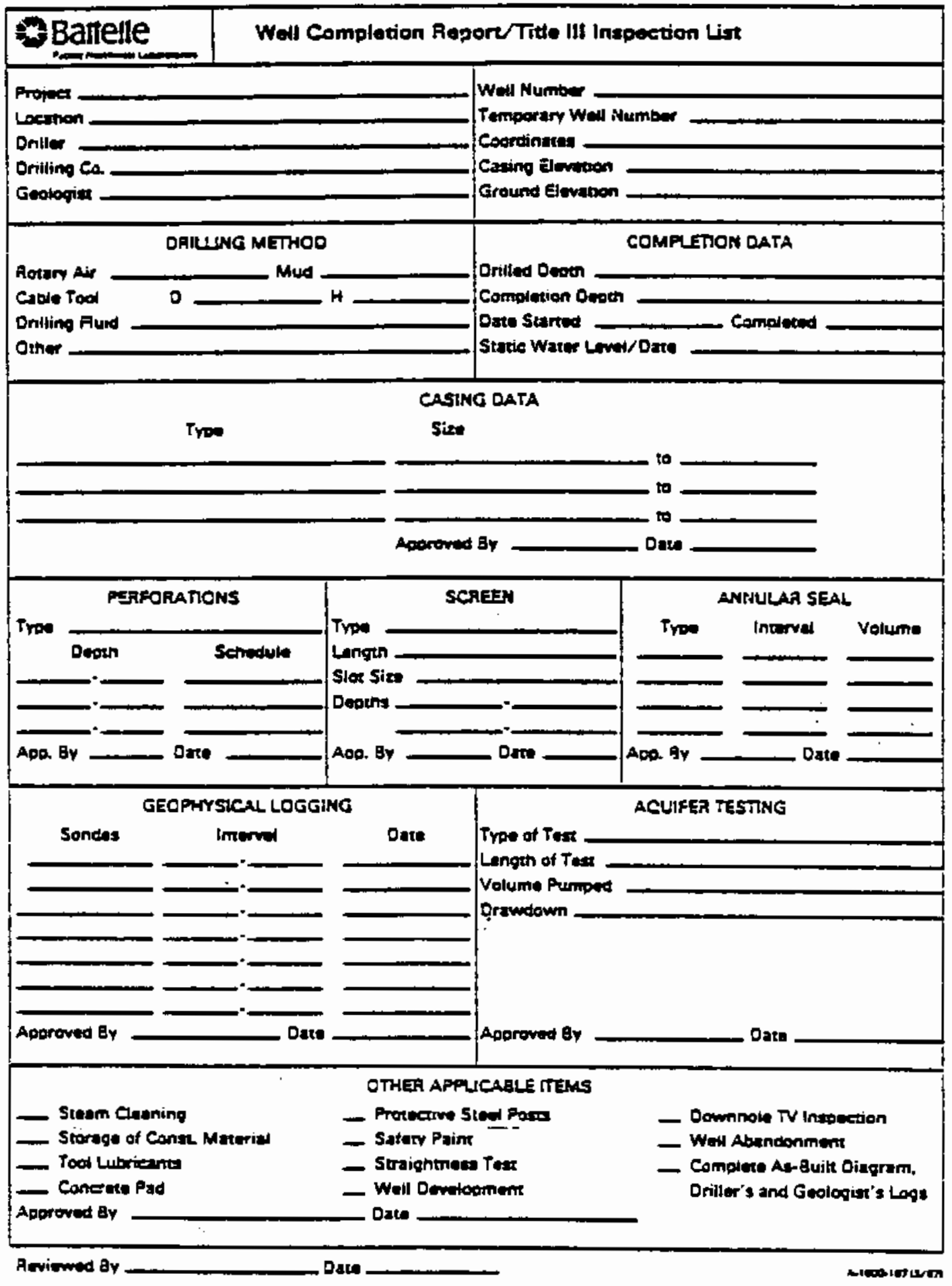




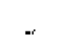


Procedure No. P-6, Rev. No. 0, Attachment 2

Page 1 of 1

.

\section{* Battelle

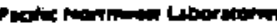

\section{AS-BUILT DIAGAAM}

Well Number

Geologist

Page of

Reviewed by

Date

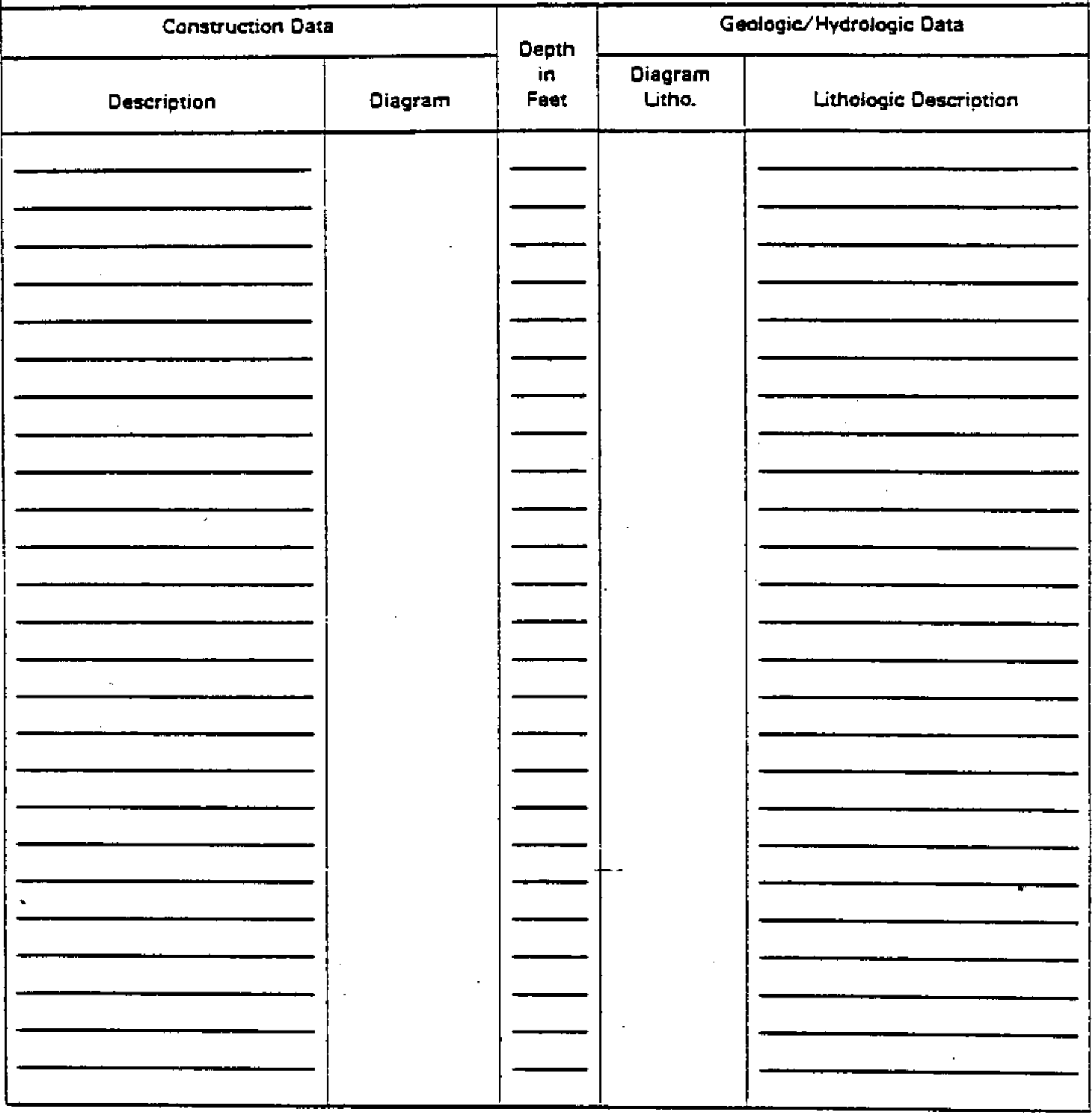


. 


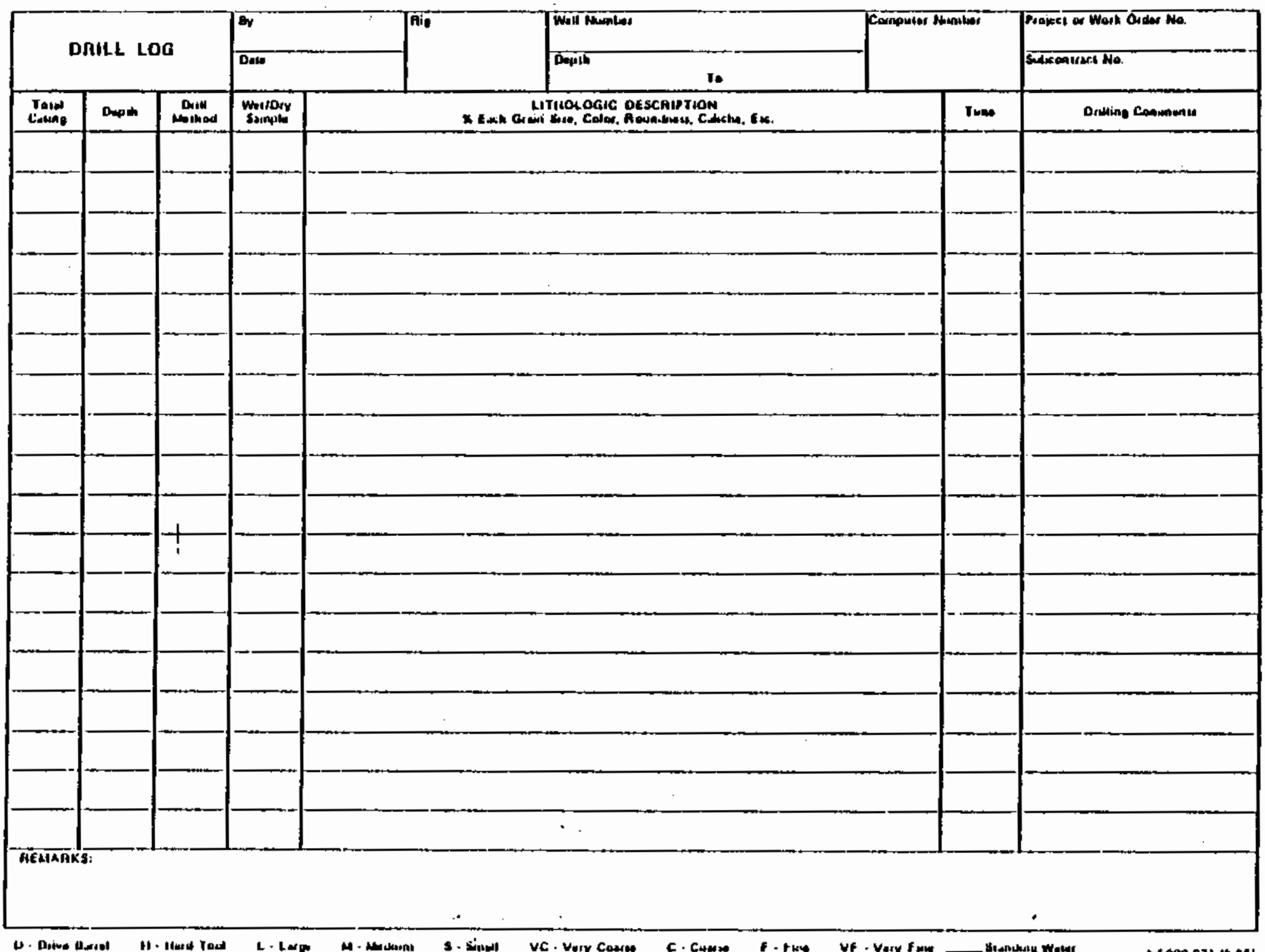





\section{COLLECTION OF SAMPLES}

A. Coliect samples at 5-ft-depth intervals and at significant changes in lithology. Use drive barrel where ever possible above the water table.

1. Drịe barrel - Have driller knock out sample from fust the very end of the drive barrel inta a clean 5-gal bucket. Note the amount of open hole when sampled, If water was added, or if the sampled was dropped, anything that might affect the sample's representativeness.

2. Hard tool - Have driller dump samples out of the bailer into a clean 5-gal bucket. Note whether the sampie is from the top or the botton of the baller, 1ndlcating how representative the sample is:

B. Collect misture samples inuediately from 5 gal bucket of every drive barrel sample. Collect sample in a moisture tin, seal the tin with whits tape, label it, and double-bag it using a plastfe bag and tape. place misture sample. in the refrigerator in back roon of sigma $V$ at the end of each shift.

C. Collect geologic samples - collect two pint jar samples from 5gal bucket. Try to get representative samples by collecting from a -vertical column within the bucket,-or roll and tumble the bucket collecting what falls in the Jar. If real busy, write the depth on the outside of the bucket or leave labeled mofsture sample in bucket untfl you can get back to it.

D. Unusual samples - Split-tube core barrel (spl1t-spoon) for recoverting relatively undisturbed samples. Note depths where drlller started and stopped oriving the sampler. After disassemily of the sampler, immedicteily wrap plastic bags around the sleeved core, taping the encs elosed. Using 
the material retained in the drive stoe for description. Placa core in. refrigerator at end of stiffit. Unpetrified Wood (for $\mathrm{C}^{14}$ ) - use tongs, wrap in aluminum foll, seal with wax. Store in refrigerator. Easalt (ior $x$-ray diffraction) - pick out best (clean and pure) basalt chips only. Nesd approx. 15 grams (7-dram vial). -

E. Each sample should be labeled directly on the Jar lid and on a sample label on the side of the jar. The following information should be included; Well no., depth, date, drilling method, and Geologist's infitals. Moisture samples should be laceled on a piece of whits tape wrapped around the side of the motsture tin. Store samples in the cardboard boxes that the sample jars came in. When a box is full, it should be sealed with duct .. tape and labeled with well no. and depth range. Protect hard tool samoles from freezing.

F. Use remainder of sampie in 5.gal bucket for sediment description. 
DESCRIPTION OF SAMPLES AND WELL CONSTRUCTION DATA

A. Sample Descriptions -- Record under It thologle-description on drill icg unless a more spectific column applies, eg. time.

Talk to the drilier and incorporats his connents on changes in the drilling conditions, and or changes in the appearance of drlll cuttings. If a major change is noted in the drlling conditions or physicai appearance of the matertal ask the drlller to collect a sample, or if it is not significant describe the new sediment from the drill cuttings. After moisture and far samples are collected, use the portion of sample left in the $5 \mathrm{gal}$. bucket for your descriptions. Include clasts too .. Jarge. to fit in Jars; note the maxlmum elast size: Note if the sediment left in the bucxet is different then that coilected in the fars. If the sample is hard tool, you may want to wash or wet sieve (using 2 num sfeve and pan) it prior to describing. Include the following in your descripticis on the drill log form:

1. Depth $\rightarrow$ ie. 4-5 (in feet).

2. Drlll method $-\mathrm{H}$ (hard tool), D (drive barrel), B (back-hoe), si (split tube), etc.

$\therefore$ 3. Wet/Ory Sample - D (dry), sk-(sifghtly woist), M (noist), VM (very moist), or $W$ (wet).

4. Lithoiogic Description -- include:

a. sample name -- ie. SANDY gRAVE - CAfITALIZE IT. This name should be based on its texture, and derived from the temary dfagram in figures 1 and 2.

b. texture - eg. I5: FP (fine pebble), 30\% VFp (very fine pejiie), 30\% ves (very coarse sand), 15\% Cs (coarse sand), and 10\% silt 


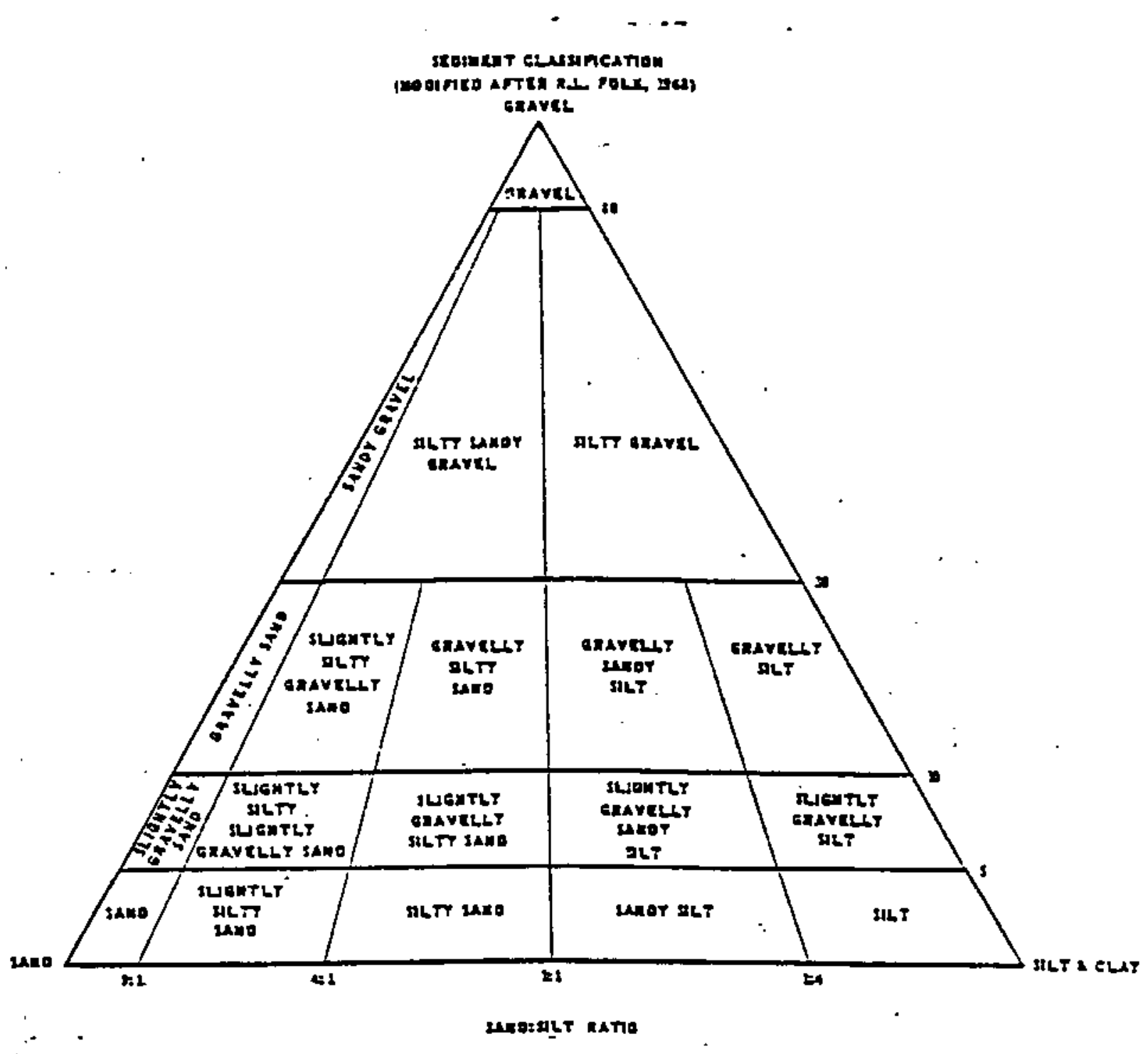

Figure 1. Temary diagram for naning of seciments, modified after RL Folk, 1968, taken from Tallman et al., 1979. 


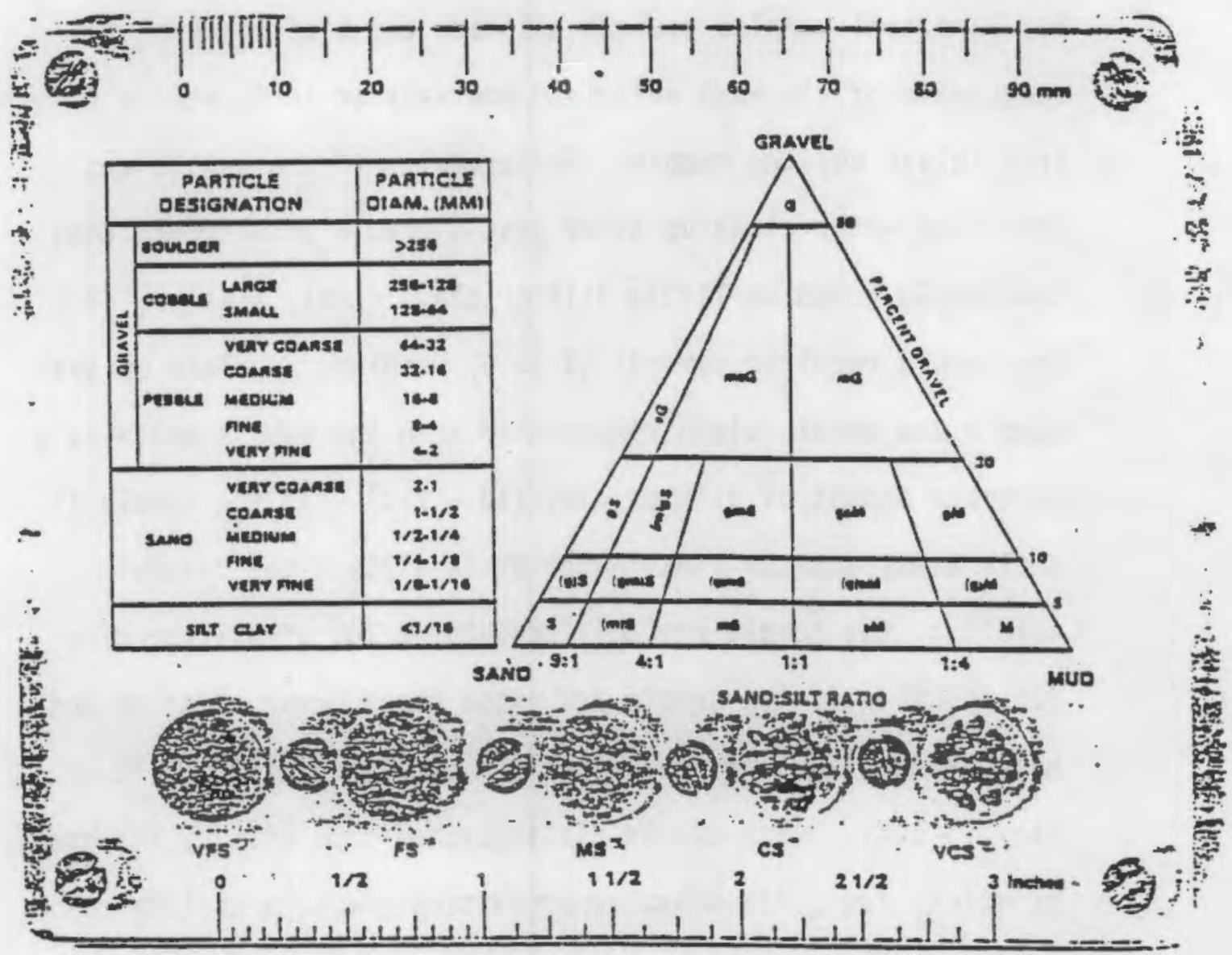

Figure 2. Enlarged photocopy of geologist's grain size chart. Note that the temary diagram uses symbols for the sedment classes. These symbols correspond to the names in ifgure 1 . 
-- Estimate the volume : of each grain size starting with the coarsest fraction first. Use the grain size charts in your geologist kit (figure 2) and the visual : charts (figure 3 ). For hard tool samples you can estimate the : of fines by the opaqueness of the wash water and how easy it is to remove ifines from (clean up) the sampie. For example, if the sample and the rinse water clean up after just a couple of washings then the sample contains little silt or clay ((10\%), however, if the sample required several ( 3 to 5 ) washings to clean up the sample and obtain clean rinse water then the sample contains a moderate amount of silt or clay (10 - 25\%). If the sample is still muddy and the rinse water still dirty after several washings, the sample probably contains a lot of silt or clay (25 - 50:). If the sample and rinse water never clear up and most of sample washes away then the sample is mostiy stit or clay $(50 \%)$. Clay can be distinguished from silt by its greasy or sticky feel, its cohesive properties (ic; 1 ts ablitity to stay in clumps or adhere to clasts), or by how long it takes the fines to settle out of the rinse water.

c. sorting -- very well sorted, well sorted, moderately sorted, poorly sorted, or very poorly sorted. The degree of sorting is determined by estimating the lower size limit of the coarsest 10\% of the detrital grains and the upper size limit of the finest 10\%. These 1 imits are then compared with the size scale to determine the number of size classes (very fine pebble, very coarse sand, coarse sand, etc.) between then. This numicer gives a measure of the degree of sorting. Refer to figure 4. 

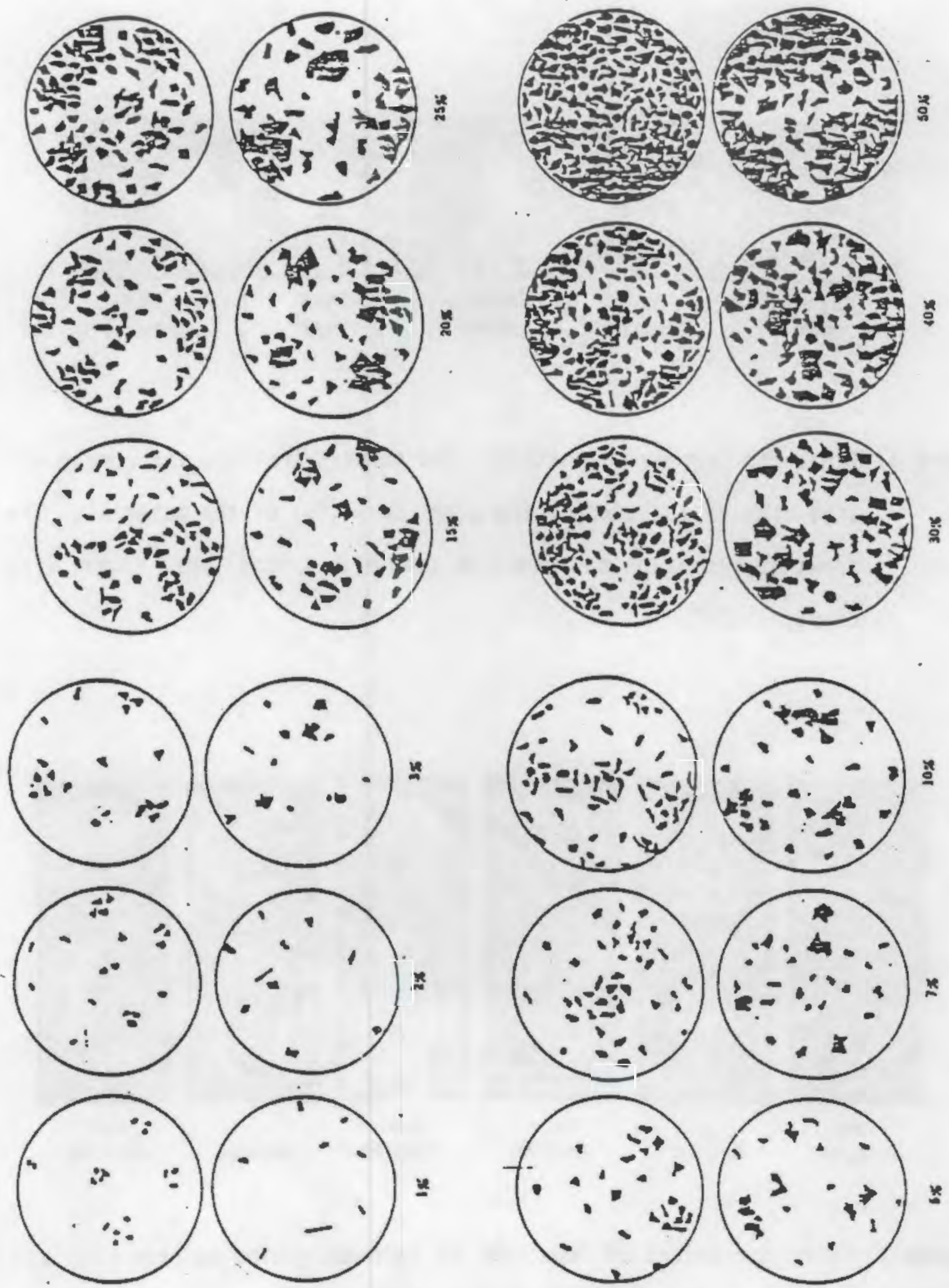

Figure 3. Charts for visually estimating percentage composition of sediments, from Compton, 1962 


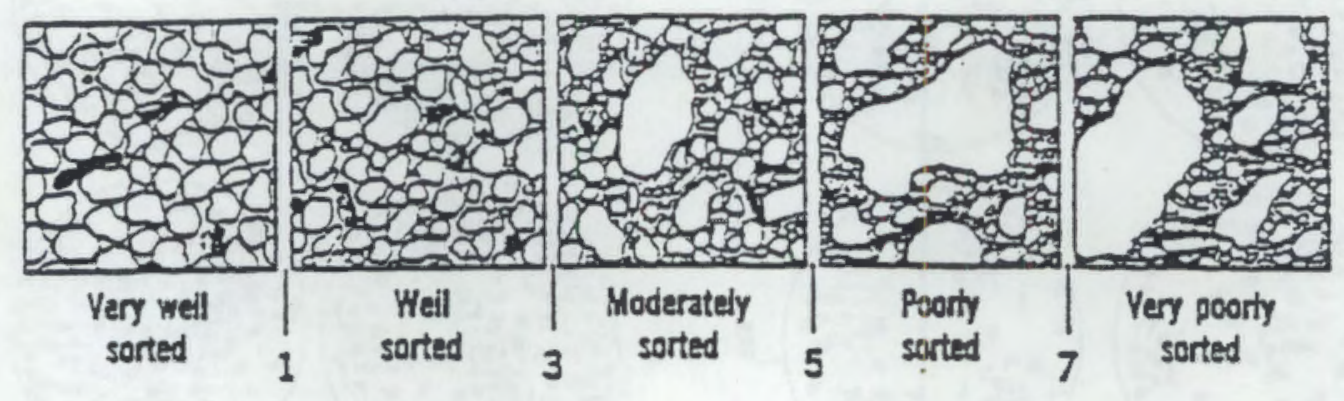

Figure 4. Terms for degrees of sorting. The numbers indicate the number of size elasses included by the great buik (80) of the material. The drawings represent sediments as seen with a hand lens. Taken from Compton, 1962.

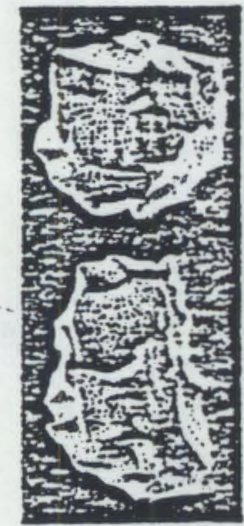

Very angular

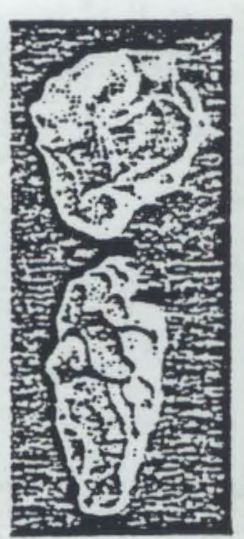

Angular

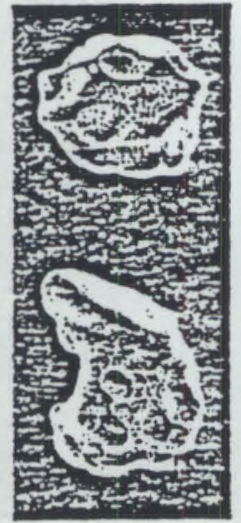

Subangular

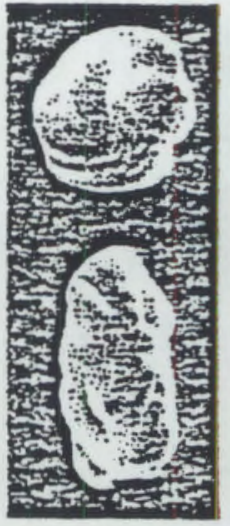

Subrounded

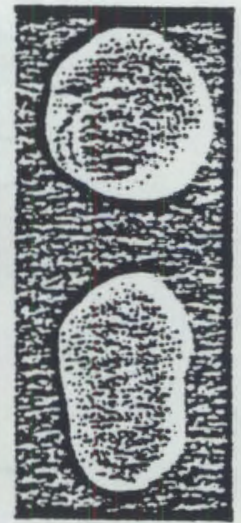

Reunded

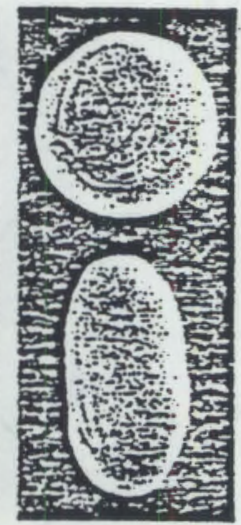

We!l rounded

Figure 5. Terms for degree of roundness of sediment grains as seen with a hand lens. Taken from Compton, 1962. 
d. gross mineralogy -- for example: Gravels are 50: basalt, 30\%

- quartztte, 10\% diorite, and 10\% other, and sands are 40\% basalt, 60\% quartz and feidspar. - Describe the gravel fraction first and then the sand fraction. Estimate the $z$ of basalt and other If thologies/mineralogy by using pebbla counts or the visual : charts (figure 3 ).

9. roundness -- Use the terns very angular, angular, sub-angular, sub-rounded, rounded, or well rounded. For exanrole: Gravei is rounded, and the sand is subrounded to angular. -- Again, describe the gravel fraction first then the sand fraction. Use the roundness charts (figure 5).

f. wet/dry color -- Use the Munsel Color Charts to delfneate both the color name and numerical value of the fine portion of the sample. If the sample is hard tool describe both the wet and dry color.

g. reaction to $10: \mathrm{HCl}$-- none, silght, moderate, strong -- check reaction on dry, fine portion of the sample.

h. Consolidation -- none, slight, moderate, well -- Estimate from the drifling rate, driller's observations (eg. hard or easy to drill, cemented or loose), and the presence of aggregates or cementation in the sample.

1. Note if there is a change in texture, color or lithology from the above sample, and approximate depth where change occurred.

j. Note unusual findings, such as unpetrified wood, smell, contamination, etc. Note the number and types of any special samples taken (moisture, wood, basalt, etc.). 
5. Time -- eg. 1340 hrs -- time sample was collected by driller, using 24-hour clock.

6. Orfling Comments -- note drilling rate, if water was added, and any problems potentially influencing the sample's representativeness. 
B. Well Construction Data -- Record under 11 thologic description on drili log unless another nore spectfic colunn applies.

1. Record $D / W$ (depth to water) and $D / B$ (depth to bottom) at the start of each shift under Drflling Couments on the drill log. Note the t1mes these measurements were taken in the time column. Use E-tape for $D / W$ and steel tape for $D / B$. Measure to nearest haif ft. and subtract top of casing (fold hold point on tape over casing to ground surface \& measure new hold point at top of casing) so that depths are recorded in feet below land surface.

2. Describe well construction activities. Include the time for dritl rig decontamination and the method used (eg; steam cleaning), Installation of casing (type, size, depth and lengths -- keep rusining totals in the Total Casing columin), time, drill method, spectfy the tool lubricants used, drill depths, drill rates (covers time only while drilling and driving casing, does not include welding time, etc.), down time (maintenance work, stand by, etc.), amounts of drilling supply water used, initial depth to water, and geophysical logging activities.

3. Well Completion $-\cdots$

a. Select well screen sizes using 50: pass through as a criterton where a natural sand pack is to be developed (steve natural materials if questionable). Refer to table 1 for the corresponding slot sizes.

b. Selection of an artificial sand pack and associated screan slot sizes is rather complicated but can be estinated by using table 2 as a general guide. If the proper selection is not 
Table 1. Sedfments, Sieve sizes and Well Screen slat sizes

Grain Size Nominclature US Standard Sfeve Opening Well Screen (Wentworth Grade Scale) Sleve Mesh Mllilmeters Inches Slot Size \#

Larger Than
Very Fine Pebbles
Very Fine Pebbles
Very Coarse Sand


TABLE 2. ESTIMATION OF ARTIFICIAL SAND PACX AND SCREEY SLOT SIZES.

\begin{tabular}{|c|c|c|}
\hline $\begin{array}{l}\text { Finest } 30 \% \text { size } \\
\text { of formation }\end{array}$ & $\begin{array}{l}\text { Formation is untform } \\
\text { and } 60 \% \text { is fine sand } \\
\text { to elay. }\end{array}$ & $\begin{array}{l}\text { Formation is highly } \\
\text { non-teniform and contains } \\
\text { silt and clay stringers }\end{array}$ \\
\hline $\begin{array}{l}\text { Coarse silt to } \\
\text { clay }(<0.063 \mathrm{~mm})\end{array}$ & $\begin{array}{l}\text { SAND PACX: } \\
\text { IOO mesh } \\
\text { SCREEN SLOT SIZE: } \\
5\end{array}$ & $\begin{array}{l}\text { SAND PACK: } \\
20-40 \text { mesh } \\
\text { SCREEN SLOT SIZE: } \\
10\end{array}$ \\
\hline $\begin{array}{l}\text { Very fine sand } \\
\text { to clay }(<0.125 \mathrm{~mm})\end{array}$ & $\begin{array}{l}\text { SAND PACX: } \\
20-40 \text { mesh } \\
\text { SCREEY SLOT SIZE: } \\
10\end{array}$ & $\begin{array}{l}\text { SAND PACX: } \\
\text { 16-30 mesh } \\
\text { 20-30 mesh } \\
\text { SCREEN SLOT SIZE: } \\
20\end{array}$ \\
\hline $\begin{array}{l}\text { Fine sand to elay } \\
(<0.25 \text { mon })\end{array}$ & $\begin{array}{l}\text { SAND PACX: } \\
\text { 16-30 mesh } \\
20-30 \text { mesh } \\
\text { 10-20 mesh } \\
\text { SCREEN SLOT SIZE: } \\
20 \text { with 16-30 or } \\
20-30 \text { mesh } \\
30 \text { with 10-20 wesh }\end{array}$ & $\begin{array}{l}\text { SAND PACK: } \\
8-12 \text { mesh } \\
\text { SCREEN SLOT SIZE: } \\
40\end{array}$ \\
\hline
\end{tabular}


obvious, then the the lead geologist or field troubie shooters should be contacted before the deteraination is made.

c. Select completion interval by using drilling specifications, geology, and geophystcat logs.

d. Note the selection and selection methods on drili log. Check with the lead geologist if selectlons are not obvious. Elevate to Field Trouble-shooters or Technical Forum for difficult problems.

4. Describe well completion activities - completion depth, periorations (type, depth and schedule), screen (type, length, slot size and depth), packer (type, size and depth), sand or gravel pack (type, Interval and volume), annular seal (type, fnterval and volume). Take frequent depth to bottom medsurements during instaliation of the sand pack and annular seals. Keep track of the depth to botton verses the depth to bottom of casing shoe, and volumes of construction materials used per finterval. Suggest that drillers not backitil too much at once. Use mud balance for checking grout or slurry. Once a specific Well Completion/Title III inspection item has been fintshed, fe. screen set, sand pack placed or seal placed, record the lower and upper interval depths and the total amount and type of material used, under Drilling Comments.

5. Describe well development, and hydrologic testing activities. 


\section{DATA MANAGEMENT}

A. Each night, make 3 coples of the drill logs (for drfller, Kalser driling engineer, and project files).

B. After completton of the project, the orlginals for each well shall be delivered to and retained by VL McGhan, Room 1230, Slgma V Building.

C. Storage of Uncontaminated Samples

1. Temporary storage (during data interpretation) -- in Container behtnd Signa $V$, or in back room of Sigina $V$ if freezing weather.

2. Permanent storage -- 2101-i4, 200 E -- fill out sample transfer record sheets, give to Anne Clarke (RHO). 


\section{REFERENCES}

Compton, R. R. 1962. Manual of Field Geology. Wiley, New York, New York.

Driscoll, F. 6. 1986. Ground Hater and Hells. Johnson Division, St. Paul, Minnisota.

Last, G. V., and T. L. Liikala. 1987. A Field Guide for Hellsite Geologists: Cable-Tool Drilling. PML-6392, Pacific Northwest Laboratories, Richland, Washington. (In press).

Tallman, A. M., K. R. Fecht, M. C. Marratt, and G. V. Last. 1979. Geploov of the Separation Areas, Hanford Site, South-Central Hashinaton. RHO-ST23, Rockwell Hanford Operations, Richland, Washington. 


\section{APPENDIX E}

\section{SAMPLING AND ANALYSIS PROCEDURES}




\section{APPENDIX E}

\section{SAMPLING AND ANALYSIS PROCEDURES}

This appendix introduces the procedures that will be used for sample collection (including well evacuation sample withdrawal methods), field measurements, sample preservation and shipment, chemical analysis, chain of custody, and quality control.

A11 sampling activities are currently performed under contract by Pacific Northwest Laboratory (PNL). United States Testing Company (UST) currently conducts sample analyses for most constituents. The procedures for laboratory analyses done by UST are documented in UST-RD-PM-9-80, Vols. 1 and 2 for radioactive constituents and UST-RD-PM-9-80, Vol. 3 for hazardous constituents (UST 1986). 
Introduction

Equipment

Test wells are sampled to determine the impact of Hanford's liquid radioactive waste disposal on the regional groundwater quality. Wells are sampled at frequencies which depend upon their location within the ground-flow network and the concentrations of radioactivity and chemical ions in the samples taken from them. Test well samples are obtained by bailing directly from the well casing or from piezometer tubes within the well casing. Air pressure is used to lift the water samples out of a piezometer tube, or a submersible pump is used in the well to bring water to the surface.

The following equipment may be needed in collecting groundwater samples:

- rubber gauntlet gioves

- sample bottles

- bucket

- temperature probe

- $\mathrm{pH}$ and conductivity meters

- well location maps and road guides

- RWP clothing

- radiation detection instruments

- electric air compressor

- truck-mounted, gasoline-powered electric generator

- pencils, masking tape

- trip sheet

- sample labels

In addition to the equipment listed above, several types of equipment may be riecessary in the collection of groundwater samples depending on the type of sample.

\section{Bailer Samples}

The following equipment may be needed in collecting a bailer sample from a test well:

- cable guide tool

- spare bailer

- truck-mounted electric winch 
Equipment (cont'd.) Organics Samples

The following equipment may be needed in collecting an organics sample from a test well:

- E-tape

- ice chest and ice

- amber glass sample bottles with teflon-lined caps

- truck-mounted organics sampling pump

- assorted tools

- sealable plastic bags

General Preparation

Load the truck with the necessary supplies in a secure and safe manner. Test all equipment prior to leaving on the trip.

Position the truck near the well for convenience and safe operation of sampling equipment. 


\subsection{SUBHERSIBLE PUHAP SAHPLES}

Introduction

Submersible Pump Sampling Procedure
Most test wells are sampled using the dedicated submersible pumps which are installed in a majority of the wells. This is the preferred sampling method because it eliminates the possibility of cross-contamination between wells.

Be sure the power switch to the 230-volt outlets is turned off.

Make sure the hose bibb is open. Never operate the pump with the outlet valve closed! Air pushed ahead of the water rising in the pipe colum must have room to escape or serious damage will occur.

Plug the power cord into one of the 230-volt outlets on the generator and into outlet at the well head.

Start the electric generator.

Turn power switch on to begin pumping process. Be sure not to handle energized power cords. If the pump does not work properly - indicated by lack of air flow out the discharge hose or the generator "lugging" down - immediately turn the switch off. After waiting a few seconds while turn the switch on and off several times rapidly, finally pausing in the "ON" position to determine if the pump has started to function properly.

Repeat this procedure several times. If the sample pump still doesn't work, it needs repaired. If the breakers or fuses on the generator go off, an electrical short in the system is indicated and repair is needed.

Begin timing after the water begins to flow from the outlet. The wells must be pumped for at least 10 minutes. (If a well "pumps dry," see "Special Procedures.")

Collect sample.

obtain sample temperature on four spaced intervals of pumping time with temperature probe.

Obtain $\mathrm{pH}$ and conductivity measurements.

Turn power switch off and then turn off generator. Unplug power cord.

Cap the sample bottle tightly, fill out the sample label with the time and date, and affix to bottle. 
Submersible Pump Sampling Procedure (cont.d.)

Special Procedures
Survey the sample bottle with a GM instrument. If the count is $>200 \mathrm{c} / \mathrm{m}$, record on trip sheet, RUP requirements apply and notify EM supervisor.

Place the sample in a secure location during transportation.

Deliver sample to appropriate laboratory for analysis and obtain signature on trip sheet of person receiving the sample.

If the weil pumps dry while waiting for a sample, it does not generally mean that no water is present. Some wells pump down after a period of time. However, a sample can still be obtained by following these steps:

- Turn off the pump when the well pumps dry.

- Hait for the well to recharge. This should be about 15 minutes, but may take as long as 30 minutes.

- Turn the pump back on. A sample can be taken inmediately, as this is water from the aquifer and not from borehole storage.
DATE ISSUED: $\quad 12-84$
580

SECTION 5.1

PAGE 5.4 


\subsection{BAILER SAMPLES}

Introduction

Bailer Sampling Procedure
Sone test welis do not produce enough water to sample by pumping, or they may contain too much sand for a purap to handle without failing. These wells are sampled using a bailer.

Place a sample bottle in the bailer and lock it in place with the weight retainer on the neck of the bottle.

Disengage the winch clutch and lower the bafler into the water.

Engage the winch clutch when bailer strikes the water surface.

Allow about 30 seconds for sample bottle to fill.

Turn on the electric winch and raise the sample to the surface, adjusting the speed of winch spool as the bailer is pulled up. Remove the sample from the bailer.

Obtain sample temperature with temperature probe and record on trip sheet.

obtain sample $\mathrm{pH}$ with $\mathrm{pH}$ meter and record on trip sheet.

Obtain sample conductivity with conductivity meter and record on trip sheet.

Cap the sample bottle tightly, fill out the sample label with the time and date, and affix to bottle.

Survey the sample bottle with a Gil instrument. If the count is $>200 \mathrm{c} / \mathrm{m}$, record on trip sheet, RWP requirements apply and notify EM supervisor.

Place the sample in a secure location during transportation.

Deliver sample to appropriate laboratory for analysis and obtain signature on trip sheet of person receiving the sample. 


\subsection{PIEZOMETER (AIR LIFT) SMIPLES}

Introduction

Piezometer Sampling (Air Lift) Procedure
Some piezometer tubes are sampled by the air lift method, in which the sample water is pushed up and out of the well by compressed air. A 1/2-in. diameter PVC tube is installed in these wells for this purpose.

Connect the compressor hose to piezometer tube.

Check the gauge on the compressed air tank. It should read in the operating range prior to the start of air lift. open the regulator valve to pressurize the hose and continue with the compressor running until water is forced out of the outlet on the side of the piezometer adapter head.

Follow pumping time indicated in the trip schedule; however, field conditions may cause some variations in the pumping time.

Rinse bucket twice before filling. Fill sample bottle from bucket.

Turn power switch off and then turn off compressor. Unplug power cord.

Obtain sample temperature with temperature probe and record on trip sheet.

Obtain $\mathrm{pH}$ with $\mathrm{pH}$ meter and record on trip sheet.

Obtain conductivity with conductivity meter and record on trip sheet.

Cap the sample bottle tightly, fill out the sample label with the time and date, and affix to bottle.

Survey the sample bottle with a GM instrument. If the count is $>200 \mathrm{c} / \mathrm{m}$, record on trip sheet, RWP requirements apply and notify EMI supervisor.

Place the sample in a secure location during transportation.

Deliver sample to appropriate laboratory for analys is and obtain signature on trip sheet of person receiving the sample. 


\subsection{PIEZOMETER (BAILING) SAMPLES}

introduction

Piezometer Sampling (Bailing) Procedure
A few piezometer tubes are sampled by the bailing method. These are piezometers which do not produce enough water to sample by air lift. The bailer used consists of a flexible rubber tube, 1-in. ID and approximately 1-1/2 to $3 \mathrm{ft} l o n g$. On one end, a brass plug is inserted and wired in place.

Bail the piezometer tube in the same manner as the weil casings are bailed, pouring the sample collected into a sampling bottle until the required amount of sample is obtained.

Obtain sample temperature with temperature probe and record on trip sheet.

Obtain $\mathrm{pH}$ with $\mathrm{pH}$ meter and record on trip sheet.

Obtain conductivity with conductivity meter and record on trip sheet.

Cap the sample bottle with a GM instrument. If the count is $>200 \mathrm{c} / \mathrm{m}$, record on trip sheet, RWP requirements apply and notify Ell supervisor.

Place the sample in a secure location during transportation.

Deliver sample to appropriate laboratory for analys is and obtain signature on trip sheet of person receiving. the sample. 


\subsection{ORGANICS SAMPLES}

Introduction

Sampling Precautions

Organics Sampling Procedure
Test wells are sampled to determine the extent and impact of Hanford's hazardous waste disposal on the regional ground-water quality. Some wells are sampled quarterly in compliance with RCRA regulations, while others may be sampled less frequently as part of a general surveillance effort. These samples are obtained with special sampling equipment designed to reduce alteration of the samples before analysis.

Do not smoke, eat, or handle any objects not necessary for sampling while performing sampling procedures. Do not sample downwind of any potential volatile organics sources such as car exhausts, open fuel tanks, etc. These could result in contaminating the sample. Note any potential sources in the area on the trip sheet if they are unavoidable. Avoid handling the tefion liner. If handling is necessary use specially prepared and protected forceps or tweezers. Do not use any liner which falls out of the cap and onto the ground.

Take water level measurement with electrical tape. CAUTION: Be sure the power switch to the 230-volt outlets is turned off!

Check to see that the hose bibb is open. Never operate the pump with the outlet valve closed! Air pushed ahead of the water rising in the pipe column must have room to escape or serious damage will occur.

Plug the power cord into one of the 230 -volt outlets on the generator and into outlet at the well head.

Start the electric generator.

Turn power switch on to begin pumping process. Be sure not to handle energized power cords. If the pump does not work properly, indicated by lack of air flow out the discharge hose or by the generator "lugging" down, immediately turn the switch off. After waiting a few seconds turn the switch on and off several times rapidly, finally pausing in the ON position to determine if the pump has started to function properly.

Repeat this procedure several times. If the sample pump still doesn't work, it needs to be repaired. If the breakers or fuses on the generator go off, an electrical short in the system is indicated and repair is needed.

Note "Special Procedures," if a we11 "pumps dry."
DATE ISSUED:

SUPEASEDES $\quad 6-81$
ISSUE DATED:
ISSUE DATED:
$6-81$ E.9
SECTON

5.5

PNL-MA-

580

PAgE 5.8 

must be pumped until three to five bore-volumes have been removed, or approximately 30 minutes.

Attach the air supply 1 ine that is attached to the filter at the rear of the organics pump console to the air compressor.

To lower the special organics pump into the well, release the slide bolt lock located on the reel drive.

Lower the pump until it is just above the dedicated submersible pump, using the handbrake to control speed and stop the reel.

Re-engage the reel lock to hold the pump at the desired depth.

Turn on the air compressor.

Adjust the pumping rate to the recomanended level of $30 \mathrm{gph}$ by using the regulator that is installed on the instrument panel. Slowly increase the regulator pressure until the pump starts cycling. Allow a few minutes for the rater discharge tube to fill and the flow meter to begin operating.

After water flows from the outlet tube, let the pump run for at least 5 minutes.

Slow the discharge to the slowest rate obtainable.

Unscrew the cap being careful not to touch the lip of the bottles or insides of the tefion liner. Also avoid touching the mouth of the discharge tap, and avoid splashing or agitating the water while the bottle is being filled.

Rinse the sample container two to three volumes with well water.

Fill the sample bottle slowly to prevent entrapment of any air bubbles. The bottle should be filled completely such that a meniscus forms.

Place the cap on immediately, turn the bottle upside down, tap it a few times and note whether there are any bubbles in the sample. If a bubble exists, discard the sample and repeat sampling including the triple rinse. If a bubble is found on the second attempt do not repeat the procedure again but note the bubble's existence on the sampie label and also notify the laboratory when it is delivered.

Turn off air compressor. 
Organics Sampling Procedure (cont'd.)
Turn power switch off and then turn off generator. Unplug power cord.

Fill out the sample label with the time and date, and affix to bottle.

Survey the sample bottle with a GM Instrument. If the count is $>200 \mathrm{c} / \mathrm{m}$, record on trip sheet, RWP requirements apply and notify EM supervisor.

Place the sample in a sealable plastic bag and then in a dark refrigerated container during transportation.

Record on trip sheet the location, EllA number, date and time of sample collection.

Deliver sample to appropriate laboratory for analys is as soon as possible and obtain signature on trip sheet of person receiving the sample. If it can not be delivered to the lab the same day, store the sample in a refrigerator which maintains a constant temperature of $4^{\circ} \mathrm{C}$. 


\subsection{HAZARDOUS MATERIALS SAMPLING}

introduction

Environmental monitoring for a wide variety of hazardous chemicals has recently been implemented at the Hanford Site. Samples to be analyzed for these materials must, in many cases, receive special treatment. Accordingly, new procedures that are specifically designed to preserve the integrity of these samples have been developed.

The procedures to be used during collection and transportation of the samples are contained in this section. All aspects of sampling, including pump operation, borehole purging, and field measurements (water leve1, pH, specific conductivity, and temperature) are described. The chain-of-custody procedures used to track and protect the samples are also included. 


\subsection{WATER-LEVEL MEASUREMENT PROCEDURE}

Introduction

Equipment

Graduated Steel Tape Method
Water-Tevel measurements are taken each time a weli is sampled, before it is purged. These measurements are taken as depth-to-water from the top of the well casing. They must be subtracted from the surveyed elevation of the casing given in Hanford Wells to obtain the elevation of the water table. The water-table elevations obtained for all wells in the sampling network during a particular sampling episode can be used to produce a contour map showing the ground-water surface at the time that the measurements were made. These contour maps can be used to help characterize the ground-water flow system and to ensure that the sampling network is adequate.

Graduated steel measuring tapes are more accurate than electrical tapes and so should be used for official measurements. However, an electrical tape can be used to determine the approximate depth to water.

The following equipment will be needed:

- steel measuring tape with attached weight

- blue carpenter's chalk

- a copy of Hanford Wells (PNL-5397)

- electrical tape

- engineer's measuring tape

- field record forms.

Chalk the 1-ft section of steel tape below the zero reading point.

Find the elevation of the measuring point and the estimated water level in Hanford Wells, or use an electric tape to find the approximate depth to water.

Lower the steel tape from the well's measuring point (marked with paint on the top of the casing) to the estimated water level. Note the amount of tape that is in the well by reading the tape at the measuring point. This value is referred to as the "hold point."

Remove the steel tape and check the wetted portion below the zero reading point.

NOTE: If the chalked portion is not wet, repeat the procedure, but allow more of the tape to go down the well (i.e., use a greater hold point).

Add the unwetted length of the chalked portion of the tape to the hold point value to obtain the depth-to-water measurement.
PNL-MA- 580

SECTION

13.1

PAge 1 of 2 
Electric Tape Method
If the chalked portion is not wet, repeat until the water level is marked on the chalked portion of the tape.

Repeat the procedure until two steel tape measurements agree within $\pm 0.05 \mathrm{ft}$.

Record the depth-to-water measurements, time of measurements, measuring device, and the name of the person taking the measurements on the field record form.

Lower the electric tape from the measuring point into the borehole until the buzzer and the light indicate contact with the water.

Mark the electric tape at the measuring point and identify the nearest graduation on the electric tape.

If the water level is deeper than the nearest graduation marked on the tape, add the difference to the depth identified to obtain the true depth to water.

If the water level is shallower than the nearest graduation marked on the tape, subtract the difference from the depth identified to obtain the true depth to water.

Record the depth-to-water measurements, time of measurements, measuring device, and the name of the person taking the measurements on the field record form.

NOTE: This measurement should be used only as an approximate depth to water, because the electric tape is less reliable than the steel tape. 
Introduction

Equipment

Sampling

Precautions
These procedures are intended for use in collecting ground-water samples that will be analyzed for hazardous chemicals.

The following equipment may be needed in the field during sampling:

- truck-mounted air compressor and generator

- bladder-pump controller box

- bladder-pump hoses (set of 2)

- extra discharge line for bladder pump

- extra discharge line for submersible pump

- Teflon bailer

- $\mathrm{pH}$ and conductivity meter

- digital thermometer

- steel measuring tape

- blue chalk

- electrical tape

- engineer's measuring tape

- a copy of Hanford Wells (PNL-5397)

- stopwatch or watch with second hand

- bucket or jug (for measuring flow rate)

- distilled water

- ziplock bags

- ice chests with ice

- plastic gloves

- aprons

- towels.

- indelible marker

- extra sample labels

- sample seals

- a copy of all relevant procedures

- sample containers with caps and liners (including extras)

- field record forms

- chain-of-custody forms

- sample-analys is request forms.

Do not smoke, eat, or handle any objects not necessary for sampling while performing sampling procedures.

Do not sample downwind of any potential sources of volatile organics such as car exhausts or open fuel tanks. These could contaminate the sample. If any such sources are unavoidable, make a note of them on the field record forms.

Leave caps on the sample containers until just before filling.

Avoid handling the Teflon bottle cap liners. Do not use any liner that falls out of the cap and onto the ground.

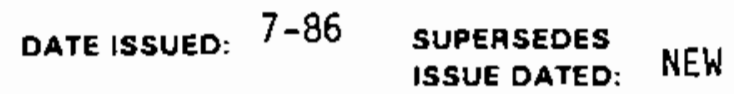

A $1400-128(5 / 60)$
E. 15
PNL.MA. 580

PAGE 1 of 6 
Sample Collection Using Submersible Pump
Wear gloves when taking samples and when handling containers, especially those with added preservative.

Take water-level measurements according to the water level measurement procedure.

Check to see that the hose bibb for the submersible pump is open.

CAUTION: Be sure the power switch to the $230-V$ outlets is turned off!

Plug the power cord into one of the $230-V$ outlets on the generator on the truck and into the outlet at the well head.

Start the electric generator.

Turn the power switch on to begin pumping process. Be sure not to handle energized power cords. If the pump does not work properly, as indicated by a lack of air flow out the discharge hose or by failure of the generator to "lug" down, turn the switch off immediately. After waiting a few seconds, turn the switch on and off several times rapidly, finally pausing in the ON position to determine if the pump has started to function properiy.

After the water begins to flow from the outlet, pump the well for the length of time indicated in Table 13.1 (or for an adjusted pumping time, as explained in Table 13.1), and until $\mathrm{pH}$, temperature, and specific conductivity stabilize. If the well being sampled is not listed in the table, pump for a minimum of 15 minutes and check for stabilization of the $\mathrm{pH}$, temperature, and specific conductivity.

NOTE: Some wells pump down after a period of time. If the well pumps dry while purging, it does not generally mean that a sample cannot be collected. A sample can still be obtained by following these steps:

1. Turn off the submersible pump when the wel? pumps dry.

2. Wait for the well to recharge. This should be about 15 minutes, but may take as long as 30 minutes.

3. Measure the depth to water using the electrical tape. Make sure that the water level is above the pump intake.

4. Turn the submersible pump back on. Collect the samples that are designated for collection with the submersible pump. $\begin{array}{ll}\text { DATE ISSUED: } 7-86 & \begin{array}{l}\text { SUPERSEDES } \\ \text { ISSUE DATED: NEW }\end{array}\end{array}$

A $1400-128: 5 / 80)$
PNL.MA. 580

E. 16
SECTION 13.2

PAge 2 of 6 
Table 13.1. Calculated Pumping Times

Well Number Calculated Time (min)

\begin{tabular}{|c|c|c|}
\hline \multirow[t]{5}{*}{ (199) } & H3-1 & 24 \\
\hline & $\mathrm{H} 4-3$ & $5^{\star}$ \\
\hline & H4-4 & $4 *$ \\
\hline & $H 4-5$ & $8 *$ \\
\hline & $\mathrm{H} 4-6 \quad \therefore$ & $5^{*}$ \\
\hline \multirow[t]{14}{*}{ (399) } & $1-1$ & 30 \\
\hline & $1-2$ & 42 \\
\hline & $1-3$ & 25 \\
\hline & $1-4$ & 34 \\
\hline & $1-5$ & $4^{\star}$ \\
\hline & $1-6$ & $6^{\star}$ \\
\hline & $1-7$ & 17 \\
\hline & $1-8$ & 29 \\
\hline & $2-1$ & 32 \\
\hline & $3-7$ & 60 \\
\hline & $3-10$ & 18 \\
\hline & $4-1$ & 35 \\
\hline & $4-7$ & 35 \\
\hline & $8-2$ & 33 \\
\hline \multirow[t]{2}{*}{ (699) } & S30-E15A & $9 *$ \\
\hline & S19-E13 & $12^{*}$ \\
\hline
\end{tabular}

* All wells will be pumped a minimum of 15 minutes, even if the calculated time is iess.

NOTE: These pumping times are based on voiding three bore-volumes of water from the well at a pumping rate of $10 \mathrm{gpm}$. To calculate an adjusted pumping time based on a field measurement of flow rate:

1. Divide the size of the container (in gal) by the number of seconds it took to fill. Multiply by 60 to get the new pumping rate (per minute).

2. Muitiply the calculated time given in Table 13.1 by 10 and then divide by the new pumping rate to get the new pumping time. 
Sample Collection Using Bladder Pump
Measure the $\mathrm{pH}$, temperature, and specific conductivity of the discharged water at least three times during purging, according to the standard procedures for measuring $\mathrm{pH}$ and conductivity. The $\mathrm{pH}$ will be considered stable when two consecutive measurements agree within $0.2 \mathrm{pH}$ units. Temperature witl be considered stable when two consecutive measurements agree within $0.2^{\circ} \mathrm{C}$. Conductivity will be considered stable when two consecutive measurements agree with in $10 \mu$ mhos. If $\mathrm{pH}$, temperature, and conductivity do not stabilize within the calculated purging time, contact the technical supervisor before collecting samples.

Enter time, date, and your initials on all sample labels.

Record information on field record form as it becomes available. The information that must be recorded on the field record forms is described in Section 13.3 (Chain-of-Custody Procedures).

Check the labels on the sample containers to determine which ones can be filled using the submersible pump. (A blue line on the right side of the sampie label indicates - that the submersible pump should not be used to fill that particular container.)

Fill the appropriate sample containers as described under "General Sampie Collection Procedure."

Attach a sample seal to each container and place it in a cooler or ice chest.

If the well has a dedicated bladder pump, turn on the air compressor and use the procedure described below. If the well does not have a bladder pump, use the procedures given for the Tefion bailer to collect the remaining samples.

Attach the compressor to the bladder-pump pressure inlet on the face of the controller panel, using the female portion of the coupling supplied.

Connect either end of the controller's red air hose to the pump supply on the right side of the control panel. Connect the other end of the controller air hose to the quick-connect nipple located in the well cap assembly.

Attach the appropriately labeled extension line to the bladder-pump discharge 1 ine.

Five to fifteen pumping cycles are required to purge the air from the bladder pump and tubing. Full water flow from the sample supply tube should then begin. After water flows from the outlet tube, run the bladder pump for at least five minutes before taking samples.
DATE ISSUED: 7-86 SUPERSEDES

ISSUE DATED: NEW
PNL-MA- 580

SECTION 13.2

E. 18

Page 4 of 6 
To reduce the water-flow rate during sample collection, turn the throttle control on the left side of the control panel in the counterclockwise direction. To increase the flow rate, turn the throttle control clockwise.

To optimize pumping efficiency for a specific wel! depth, refer to the pump manufacturer's operating instructions.

Sample Collection Using Tefton Baiter

Genera] Sample Collection Procedure
Unclasp the metal bajler from the winch line and replace it with the Tefion bailer.

Disengage the winch clutch and slowly lower the bailer into the water.

Engage the winch clutch when the bailer strikes the water surface.

Allow about 30 seconds for the sample tube to fill.

Turn on the electric winch and slowly raise the Teflon bailer to the surface.

Lower and rinse the bailer twice before collecting a sample.

Unscrew the cap of the sample container, being careful not to touch the lip of the bottle or the inside of the Tefion liner. Avoid touching the mouth of the Teflon bailer.

Unclasp the Teflon bailer.

Pour the water from the bajler into the sample container slowly to prevent trapping any air bubbles. Avoid splashing or agitating the water while the sample container is being filled.

Unscrew the cap from the sample container, being careful not to touch the lip of the bottle or the inside of the Teflon liner. Also avoid touching the mouth of the discharge line.

Fill the sample bottle slowly by placing the outlet tube against the inner side of the sample bottle to prevent trapping any air bubbles. Avoid splashing or agitating the water while the bottle is being filled.

NOTE: For those bottles requiring no headspace, the bottle should be filled completely so that a meniscus forms. Cap the bottle immediately, turn it upside down, tap it a few times and check for air bubbles in the sample. If a bubble exists, discard the sample and repeat the sampling procedures until an
PNLL-MA. 580

E. 19
SECTION 13.2

PAGE 5 of 6 
Teflon Bajler

Cleaning

Procedure air-free sample is obtained. There is an exception to this if the bottle to be filled contains a preservative, in which case only one attempt will be made to obtain an air-free sample.

As each container is filled, attach a sample seal to it and place it in a cooler or ice chest.

Survey the sample container with a $G M$ instrument. If the count is greater than $200 \mathrm{c} / \mathrm{m}$, record it on the field record form and use Radiation Work Procedure 318-EE-1. Contact the EM Supervisor for instructions concerning where the sample is to be taken.

Turn off the air compressor.

Turn the power switch off and then turn the generator off. Unplug the power cord.

Complete the chain-of-custody and sample-analys is request forms.

Deliver the sample to the appropriate laboratory for analysis as soon as possible, following chain-of-custody procedures. If it cannot be delivered to the lab the same day, store the sample in a refrigerator located inside a locked building or within a secured area. The refrigerator must maintain a constant temperature of $4^{\circ} \mathrm{C}\left(39^{\circ} \mathrm{F}\right)$. Leave the cooler to be used when the samples are delivered.

Wash the inside and the outside of the bailer with a mild mixture of dish soap and water.

Rinse the bajler twice with tap water.

Store the bailer in a sealable plastic bag between uses. 
Introduction

Delivery Procedures
To ensure the integrity of the samples from the time of collection through data reporting, the history of the custody of each sample will be documented according to these procedures. A sample is considered to be under a person's custody if it is in any of the following states: 1) in his physical possession; 2) in his view after he has taken possession; 3) secured by him so that no one can tamper with the sample; or 4) secured by him in an area which is restricted to authorized personnel. Anyone having custody of samples must comply with the procedures described below.

Sample Labels

Fill out and affix gummed paper labels to the sample containers prior to or at the time of sample collection. The label to be used is shown in Exhibit 13.1. The well number noted on the label identifies the well location where the sample was collected.

\section{Sample Seals}

Attach gummed paper seals to the samples immediately upon sample collection, before the samples leave your custody. Attach the seal in such a way that the sample cannot be opened without breaking the seal.

\section{Field Record Form}

Record (in black ink) all pertinent information about each sample collected on a field record form and insert into a logbook. It will be a bound book with consecutively numbered pages. An example field record form is shown in Exhibit 13.2.

Chain-of-Custody Form

A chain-of-custody form will accompany all samples from the time they are collected until they are disposed of after analys is and reporting. A single form will be used for as many samples as possible. The form to be used is shown in Exhibit 13.3. Each person who handles the sample and signs the form witl return a copy of the form to the company contact whose name appears on the top line.

\section{Sample-Analys is Request Form}

UST requires that a sample-analys is request form accompany all samples delivered to the lab. The form to be used is shown in Exhibit 13.4. The field portion of the form will be completed by the sample collector; the laboratory portion will be completed by laboratory personnel.
PNL-MA- 580

E.21
SECTION 13.3

PAGE 1 of 7 
Laboratory Acceptance Procedures

Receiving Department
You will nomally deliver samples directly to the laboratory on the day of collection. If they cannot be delivered on the day of collection, you must store them in a refrigerator in a locked building. (No shipping of the samples will be necessary, due to the lab's proximity to the site.) Al) samples will be accompanied by a chain-of-custody form and a sample-analysis request form. Deliver samples only to authorized laboratory personnel.

The chain-of-custody does not end at the laboratory door, and therefore the laboratory must ensure the continuity of its record by following these procedures:

Remove the sample cooler from the delivery vehicle and bring it into the receiving area.

Check the sample cooler for any obvious damage.

Sign the chain-of-custody and sample-analysis request forms.

If you transfer custody of the cooler to one or more intermediates before it is delivered to the Sample Custodian, the chain-of-custody form must reflect every change of custody.

(sample number) - company code

(list of analyses)

(well number)

(bottle preparation)

SAMPLE SIZE :

LAB :

DATE/TIME :

COLLECTOR:

Exhibit 13.1. Sample Label

PNL-MA. 580

E. 22
SECTION 13.3

PAGE 2 of 7 
Well Number

Oate

Pumping Time: Precalculated

New

Submersible (Time On)

Bladder (Time On)

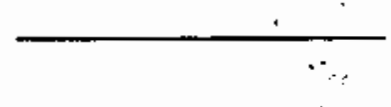

SAMPLES COLLECTED SAMPLE NO.
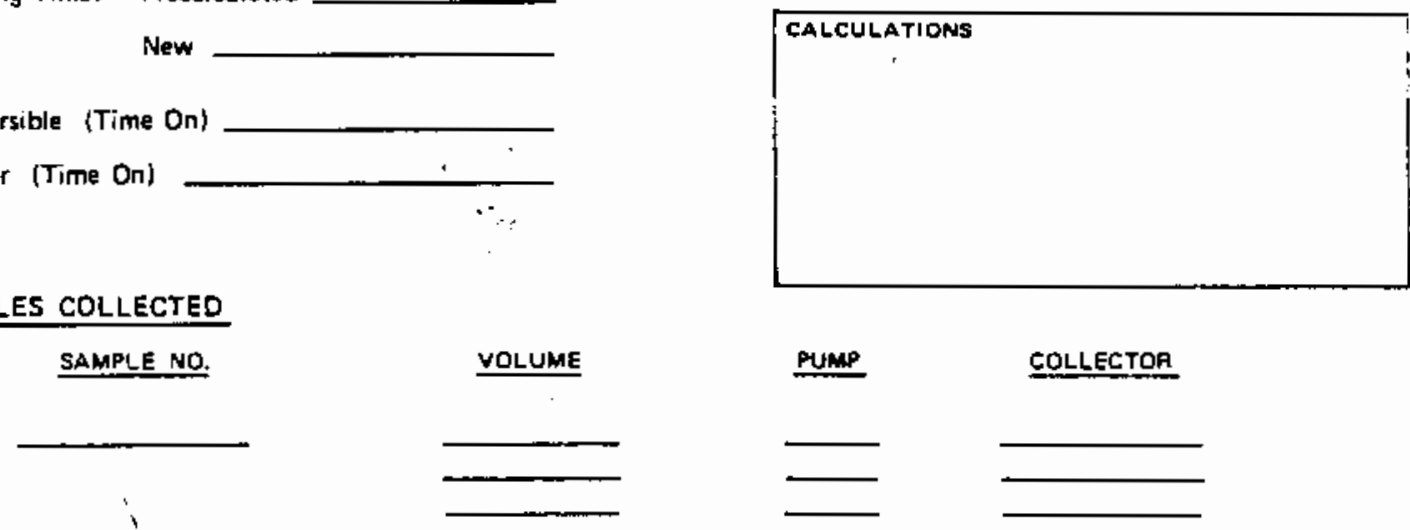

(E - tape I
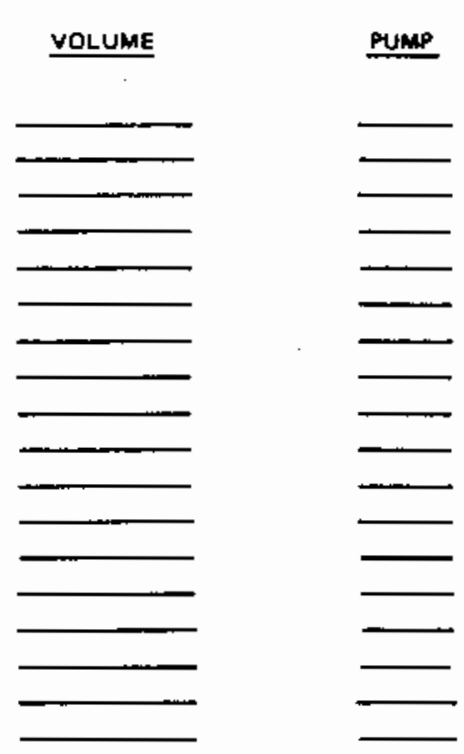

COLLECTOA

FIELD MEASUREMENTS

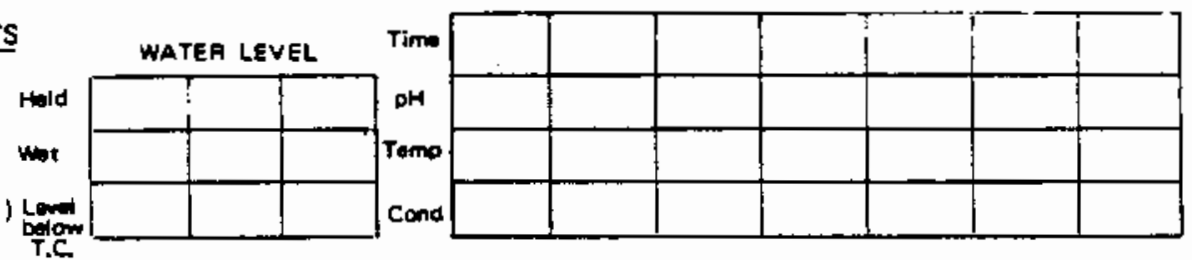

FIELO OESERVATIONS

Data Recorded By

Chain-Or-Custody Form No.
Data Checked By

Laboratory Fecord Book No.

Page No.

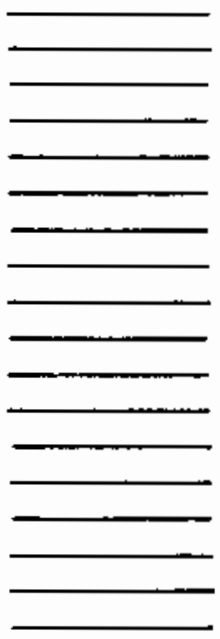




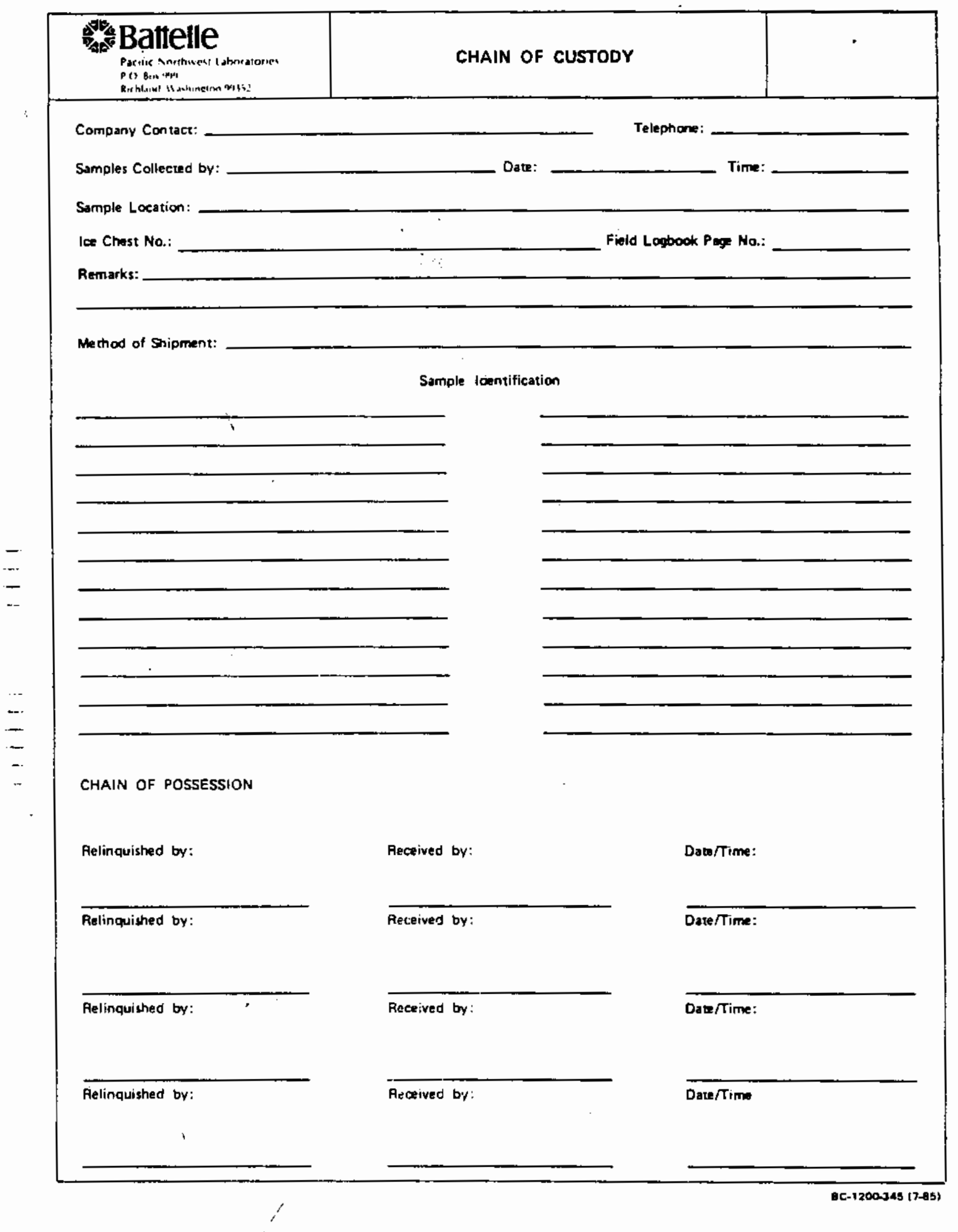

EXHIBIT 13.3. Chain-of-Custody Form

DATE ISSUED: $7-86$
NEW
PNL-MA- 580

E. 24
SECTION 13.3

PAge 4 of 7 
Pacteic Northweat Ldoocatory

$P 0$ Box 999

WA

99352
United States Tasting Company. ine.

2800 George Washingron way

Richland Ha 99352

Received by

Ticle

Dace

Time

Company Contace

\begin{tabular}{l|l|l|l|l|l|}
\hline 3 & 5 & 6 & 7 & 8
\end{tabular}

CHAIN OF CUSTODY NO.

SAMPLE ID

UST SAMPLE ID

COM MEN T S

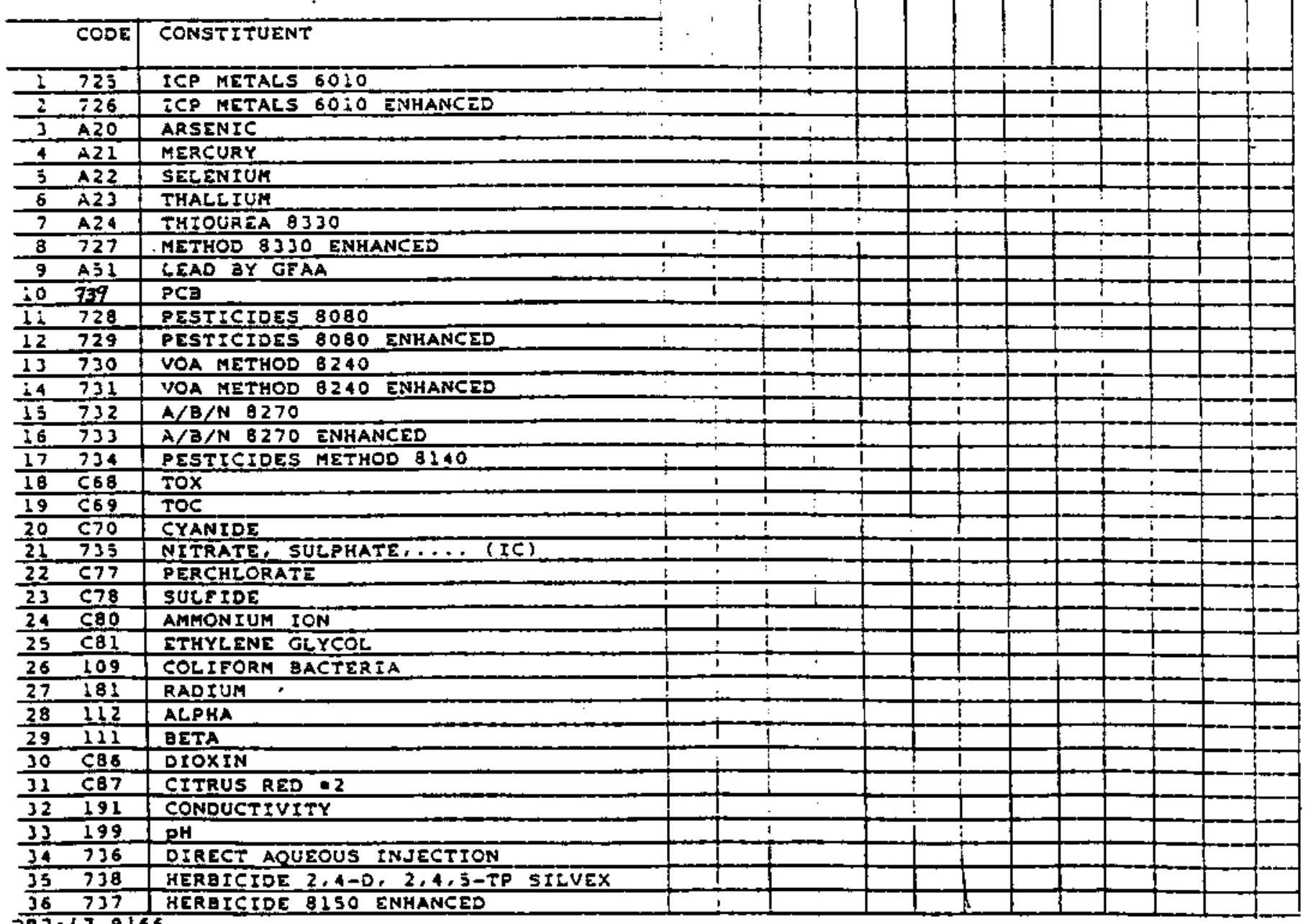
Dर2:17.9166

DATE ISSUED: $7-86$
PNL-MA. 580

SECTION 13.3

PAgE 5 of 7 
When the sample cooler is delivered to you (or your designated alternate), sign the chain-of-custody form.

Then return one copy to the sender and retain the remaining copies. The sample cooler is now your responsibility (or that of your designated alternate). You must now log the samples in.

Examine the sample coolers. On the sample log-in form (Exhibit 13.5 ), record

- presence/absence of custody seal(s) on the samples

- condition of custody seal(s).

Open the sample coolers, examine the sample documents, and record on the sample log-in form whether chain-of-custody form(s) are present.

Remove sample containers and record on sample log-in form:

- condition of samples (intact, broken, leaking, etc.)

- presence/absence of sample labels and sample seals

- any discrepancy with chain-of-custody form(s).

If discrepancies are found, contact the sender for clarification.

Once a 11 samples have been properly logged in, send a copy of the sample log-in form to the company contact named on the chain-of-custody form.

Use an internal numbering system for identification of all samples.

Assign internal numbers to the samples and record the numbers on the sample log-in form alongside the corresponding sample number assigned by the collector.

Place the properly labeled sample containers in the secure storage area. 
DATE :

TIME :

COOLER ID :

CLIENT SAMPLE ID:

UST-RD ID :

NUMBER OF BOTTLES IN COOLER

IS CHAIN OF CUSTODY FORM PRESENT? :

CHAIN OF CUSTODY NUMBER

IS SAMPLE ANALYSIS REQUEST FORM PRESENT? :

* is the custody seal on the cooler intact? :

* are the custody seals on the bottles intact? :

* do the sample labels agree with the chain of Cystody sheet? :

* Do the sample labels agree hith the sample analysis REQUEST SHEET? :

* If anSwer is 'no', please explain belon in detail.

SAMPLES LOGGED IN BY:

REVIEHED BY SAMPLE CUSTODIAN:

Govind Rao

Exhibit 13,5. Sample Log-In Form

PNL-MA- 580

SECTION 13.3

E. 27

PAGE 7 of 7 


\subsection{TEMPERATURE MEASUREMENT PROCEDURE}

Introduction

Equipment

Temperature Measurement Procedure
Temperature measurements are taken during and after purging of the well, just prior to sample collection. Measurements taken during purging are used to help ensure that the well bore has been sufficiently evacuated, as indicated by stabilization of the temperature. (The $\mathrm{pH}$ and conductivity are measured at the same time for the same reason.) The temperature is considered stable when two consecutive measurements agree within $0.2^{\circ} \mathrm{C}$. The final temperature measurement is taken just prior to sampling and is recorded as an analytical value for the sample. The digital thermometers are regularly checked against a standard thermometer for accuracy.

The following equipment will be needed:

- digital thermometer

- field record forms.

Turn on the digital thermometer. Make sure that the switch is positioned so that the measurements will be in degrees centigrade.

Place the probe into the stream of water being discharged from the pump.

The temperature is indicated by a flashing display, which will normally fluctuate for a few seconds. Wait until fluctuation ceases (i.e., until the same temperature is indicated on three consecutive flashes), and then record the temperature on the field record form. 
Introduction

Equipment

Conductivity

Calibration

Procedure
Conductivity measurements are taken during and after purging of the well, just prior to sample collection. Measurements taken during purging are used to help ensure that the well bore has been sufficiently evacuated, as indicated by stabilization of the conductivity. (The $\mathrm{pH}$ and temperature are measured at the same time for the same reason.) Conductivity is considered stable when two consecutive measurements agree within 10 umhos. The final conductivity measurement is taken just prior to sampling and is recorded as an analytical value for the sample. The conductivity meter should be calibrated once a day, before it is taken to the field to begin sampling.

The following equipment will be needed:

- conductivity meter

- distilled or deionized water

- small screwdriver

- standard solution

- field record forms.

Internal Standard

Empty the sample cup on the meter. Rinse it out with distilled or deionized water. Ory the cup thoroughly.

Turn the range switch to TEST.

Press the OPERATE button.

Use the small screwdriver to adjust the CALIBRATE until the meter or display reads 8 .

NOTE: This is to be done as a rough calibration or battery check.

\section{Standard Solution}

Empty the sample cup. Rinse it out with distilled or deionized water.

Fill the cup with standard solution.

Turn the MODE switch to conductivity.

Turn the RANGE selector switch to the correct range for the standard solution.

Press the OPERATE button. 
Conductivity Measurement Procedure
Use the smail screwdriver to adjust the CALIBRATE unti] the meter or display reads the solution value.

NOTE: Do not return the conductivity standard solution to the container. Always discard it.

Check that the conductivity meter is properly calibrated by using the internal standard.

Remove the cap from the sample cup.

Rinse the cup with the water to be tested and dump it several times. Then fill the cup with the sample.

Turn the conductivity RAMGE switch to the correct range.

Range in umhos

$$
\begin{array}{ll}
\times 1 . & 0-10 \\
\times 10 . & 0=100 \\
\times 100 . & 0=1,000 \\
\times 1,000 . & 0=10,000
\end{array}
$$

Turn the MODE switch to CONDUCTIVITY.

Press the OPERATE button on the side of the meter.

Read the conductivity on the digital display to the nearest umho and record on field record form. Multiply the meter reading times the scale factor to obtain the correct placement of the decimal point.

After taking the reading, dump the sample and refill the cup with distilied water.

$$
\text { Replace the cap. }
$$




\section{Introduction}

Equipment

$\mathrm{pH}$ Calibration Procedure
Measurements of the $\mathrm{pH}$ are taken during and after purging of the well, just prior to sample collection. Measurements taken during purging are used to help ensure that the well bore has been sufficiently evacuated, as indicated by stabilization of the $\mathrm{pH}$. (Conductivity and temperature are measured at the same time for the same reason.) The $\mathrm{pH}$ is considered stable when two consecutive measurements agree within $0.2 \mathrm{pH}$ units. The final $\mathrm{pH}$ measurement is taken just prior to sampling and is recorded as an analytical value for the sample. The $\mathrm{pH}$ instrument should be calibrated once a day, before it is taken to the field for sampling.

The following equipment witl be needed:

- $\mathrm{pH}$ meter

- distilled or deionized water

- smali screwdriver

- buffer solutions

- field record forms.

Wash the meter's sample cup with distilled water.

Fill the cup with 4.0 buffer solution.

Turn the MODE switch to $\mathrm{pH}$.

Press the OPERATE button. Use the small screwdriver to adjust the ZERO to make the display read $4.00 \mathrm{pH}$ on the upper meter scale or on the digital display.

Discard the buffer solution. Wash the cup twice with distilled or deionized water.

Fill the cup with 10.00 buffer solution.

Adjust the SLOPE to make the display read $10.00 \mathrm{pH}$.

Rinse the cup again and refill it with $4.0 \mathrm{pH}$ buffer solution. Recheck the $4.0 \mathrm{pH}$ value and adjust the ZERO if necessary.

Single Buffer Calibration.

After the SLOPE is calibrated the first time each day, it may be left alone for subsequent calibration. Recheck the slope as desired. 
pH Measurement Procedure
Calibrate with a buffer solution that is close to the range of interest. If the process $\mathrm{pH}$ is from 6.0 to 9.0 , use a 7.0 buffer solution for single-point calibration.

With the buffer solution in the cup, recalibrate only the STO to make the display read the desired value.

NOTE: Do not return buffer solutions to their containers. Always discard them.

Ensure that"the $\mathrm{pH}$ instrument is properly calibrated.

Remove the cap from the sample cup.

Rinse the cup with the water to be tested several times. Then fill the cup with the sample.

Turn the MOOE selector switch to $\mathrm{pH}$.

Press the OPERATE button on the side of the meter.

Read the $\mathrm{pH}$ on the upper meter scale or on the digital display to the nearest tenth of a unit. Record the value on the field record form.

Dump the sample and refill the cup with distilled water. Replace the cap. 
Procedure for Disposal of Excess Ground Water Samples, P-1

for RCRA Compliance/Hazardous Materials Monitoring

\subsection{OBJECTIVES}

The objective of this procedure is to establish a method for disposing of ground-water samples that remain after all laboratory analyses are completed. The procedure applies to samples generated in and before FY 1986. The FY 1987 contract for sample analysis with U.S. Testing (UST), will provide for disposal by UST.

\subsection{REQUIREMENTS}

Adherence to the procedure for disposal of ground-water samples shall assure that disposal of these samples conforms to all washington State regulations dealing with hazardous wastes.

\subsection{COMPLIANCE WITH REGULATIONS}

Criteria for unrestricted disposal of ground-water samples have been complied and communicated to this project by PNL Laboratory Safety staff members Jeene Hobbs and Manford Leale. None of the sample concentrations reviewed would be designated as regulated hazardous waste under Washington State's dangerous waste regulations in WAC 173-303 . (D)

Compliance with waste regulations will be re-checked if any major changes occur in concentration levels. PNL Laboratory Safety shall be contacted to provide special disposal instruction in case the contents of any bottles exceed al lowable concentrations of radionuclides or hazardous materials.

\subsection{DISPOSAL OF SAMPLES}

Personnel from UST will return any bottles containing remaining ground water to PNL, along with the completed chain-of-custody forms. If the entire sample is used, UST will dispose of empty sample containers, and return chajn-of-custody forms to PNL.

(a) Memo from Jeene Hobbs to Jan Carlile, dated 12/09/85.

Memo from Manford Leale to Marcia Walter, dated 01/23/86.

(b) Memo from Jeene Hobbs to Marcia Walter, dated 01/31/86.

\begin{tabular}{|c|c|c|c|}
\hline \multicolumn{2}{|c|}{ 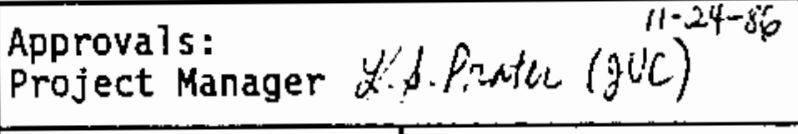 } & \multicolumn{2}{|c|}{ QA Rep , XKR Gahl $1 / 14 / 8$} \\
\hline $\begin{array}{l}\text { Procedure No: } \\
\text { P-1 }\end{array}$ & $\begin{array}{c}\text { Revision No: } \\
0\end{array}$ & $\begin{array}{l}\text { Date Issued: } \\
11-24-86\end{array}$ & Page 1 of 2 \\
\hline
\end{tabular}


Procedure for Disposal of Excess Ground-Water Samples, $P-1$

Disposal of ground-water samples will be carried out by PNL.

Ground-water samples that can be disposed into the sewer system will be disposed of in a PNL laboratory facility (drain). PNL Laboratory Safety shall be contacted to provide special disposal instruction in case the contents of any bottles exceed allowable concentrations of radionuclides or hazardous materials.

Empty bottles will be disposed in a trash container.

Completed chain-of-custody forms will be filed as project records.

\begin{tabular}{|c|c|c|c|}
\hline $\begin{array}{c}\text { Procedure No: } \\
\text { P-1 }\end{array}$ & $\begin{array}{c}\text { Revision No: } \\
0\end{array}$ & $\begin{array}{c}\text { Date Issued: } \\
11-24-86\end{array}$ & Page 2 of 2 \\
\hline
\end{tabular}




\subsection{OBJECTIVE}

The objective of this procedure is to establish a standardized method for the receipt, entry into the PNL data base, verification, and review of data from United States Testing (UST), the primary analytical laboratory. Data are received from UST in both magnetic media and hard copy form.

\subsection{REQUIREMENTS}

The incoming data from the primary laboratory shall be checked to assure their accuracy and completeness. Entry of the data into the data base will be verified against the raw data from the laboratory.

\subsection{DATA HANOLING, VERIFICATION, AND REVIEW}

Magnetic tapes, two types of data listings, and raw data sheets from the laboratory are delivered to PNL by UST personnel. One of the listings is a summary report, which is used for data review. The other listing is an image of the file as it appears on magnetic tape, which is used for verification of the contents of the tape.

The sunmary report is reviewed by the Sample Analysis Task Leader to find results which are greater than detection limits, blanks which have values above detection limits, or any suspect results. All discrepancies shall be investigated and resolutions documented.

The data file is copied from magnetic tape to the disk on the wrax computer. A listing of this input file is compared with the listing from UST to verify the contents of the tape. (This is not a detailed comparison, but a check for the correct number of records, the proper date, etc.)

Two programs are run to check the input file and read it into the data base:

The UPORES (update results) program is used to process the file of data into the data base. The program reads the file, checks the file for format errors, and generates a report which lists rejected data. Examples of circumstances which cause rejection of data include improper well or constituent codes, no sample date associated with the analysis value, or receipt of internal UST QC data that is not intended to be included in the PNL data base. The output from the UPDRES program is reviewed, and any unexplained rejects are investigated.

\begin{tabular}{|c|c|c|c|}
\hline \multicolumn{2}{|c|}{$\begin{array}{l}\text { Approvals: } \\
\text { Project Manager }\end{array}$} & QA Rep $Q$ & $-26-87$ \\
\hline $\begin{array}{l}\text { Procedure No: } \\
\text { P-2 }\end{array}$ & Revision No: & $\begin{array}{c}\text { Date Issued: } \\
3 / 30 / 87\end{array}$ & Page 1 of 3 \\
\hline
\end{tabular}


Procedure for Data Handling and Verification, P-2

The ANATREND (analytical trend) program reads the file generated by UPDRES, does trend-checking, and generates a report of results that have been stored in the data base. The trend-checking program flags

- values that are outside 2.09 times the standard deviation of the

linear regression curve for each sampling point.

The report generated by ANATREND is used to verify values recorded in the database against the raw data sheets from UST by a designee of the Sample Analysis Task Leader. This is accomplished by following methods described in American National Standard ANSI/ASQC 21.4-1981 "Sampling Procedures and Tables for Inspection by Attributes" at an Acceptable Quality Level (AQL) of $1 \%$. Pertinent information from Tables I and IIA from this document are included in Attachment 1 of this procedure. The procedure to be followed in data verification by sampling inspection consists of the following steps:

1. Determjine the number of records that were entered into the database from the UST data tape. This number is printed at the end of ANADAT.RPT. This is the Lot or Batch Size.

2. Determine the Sample Size from Attachment 1 for the lot or Batch Size.

3. Determine the sampling interval (SI) as the whole number portion of (Lot or Batch Size)/(Sample Size).

4. Mark every SI-th record on the ANADAT.RPT computer listing that is produced from the tape.

5. Check every record marked in step 4 against its laboratory data sheet value and initial each value checked on ANADAT.RPT.

6. Count the number of checked records that are nonconforming; that is the number that are incorrect in the database.

7. Determine the Acceptance and Rejection numbers from Attachment 1 for the Sample Size that has been used.

8. If the number of nonconformances is less than or equal to the Acceptance number sign the ANADAT.RPT listing as accepted and enter the initials of the verifier into the data base for each record from that tape.

\begin{tabular}{|c|c|c|c|}
\hline $\begin{array}{c}\text { Procédure No: } \\
\text { P-2 }\end{array}$ & $\begin{array}{c}\text { Revision No: } \\
1\end{array}$ & $\begin{array}{c}\text { Date Issued: } \\
3 / 30 / 87\end{array}$ & Page 2 of 3 \\
\hline
\end{tabular}


Procedure for Data Handling and Verification, P-2

If the number of errors is greater than or equal to the Rejection number, verify every number in the ANADAT.RPT listing and report the results to the Statistical and Qualitative Data Evaluation Task

. Leader for further action as outlined in ANSI/ASQC Z1.4-1981.

If discrepancies or errors are discovered, they shall be brought to the attention of the Sample Analysis or Data Handling Task Leader (or designee) who shall determine any corrective action. If data are changed in the data base, the new results are automaticaliy included in subsequent ANATREND runs. The most recent value that was changed remains stored in the file in a separate location.

Field data are hand-entered by designated data handling staff. These . data are verified by comparing field notes with the data listing.

Any input errors in field data shall be corrected. When field data are correctly entered, the person verifying the data shall sign and date the listing and submit it to the Data Handing Task leader. This verification listing shall be treated as a project record.

If the laboratory provides a corrected result on magnetic tape, the record will automaticaliy be modified in the data base and included in subsequent ANATREND runs. Corrected results are marked as "modified" in the data base.

\begin{tabular}{|c|c|c|c|}
\hline $\begin{array}{c}\text { Procedure No: } \\
\text { P-2 }\end{array}$ & $\begin{array}{c}\text { Revision No: } \\
1\end{array}$ & $\begin{array}{c}\text { Date Issued: } \\
3 / 30 / 87\end{array}$ & Page 3 of 3 \\
\hline
\end{tabular}


Procedure No. P-2, Rev. No. 1 ATTACHMENT 1

Page. 1 of 1

\section{SAMPLING PLANS FOR NORMAL INSPECTION}

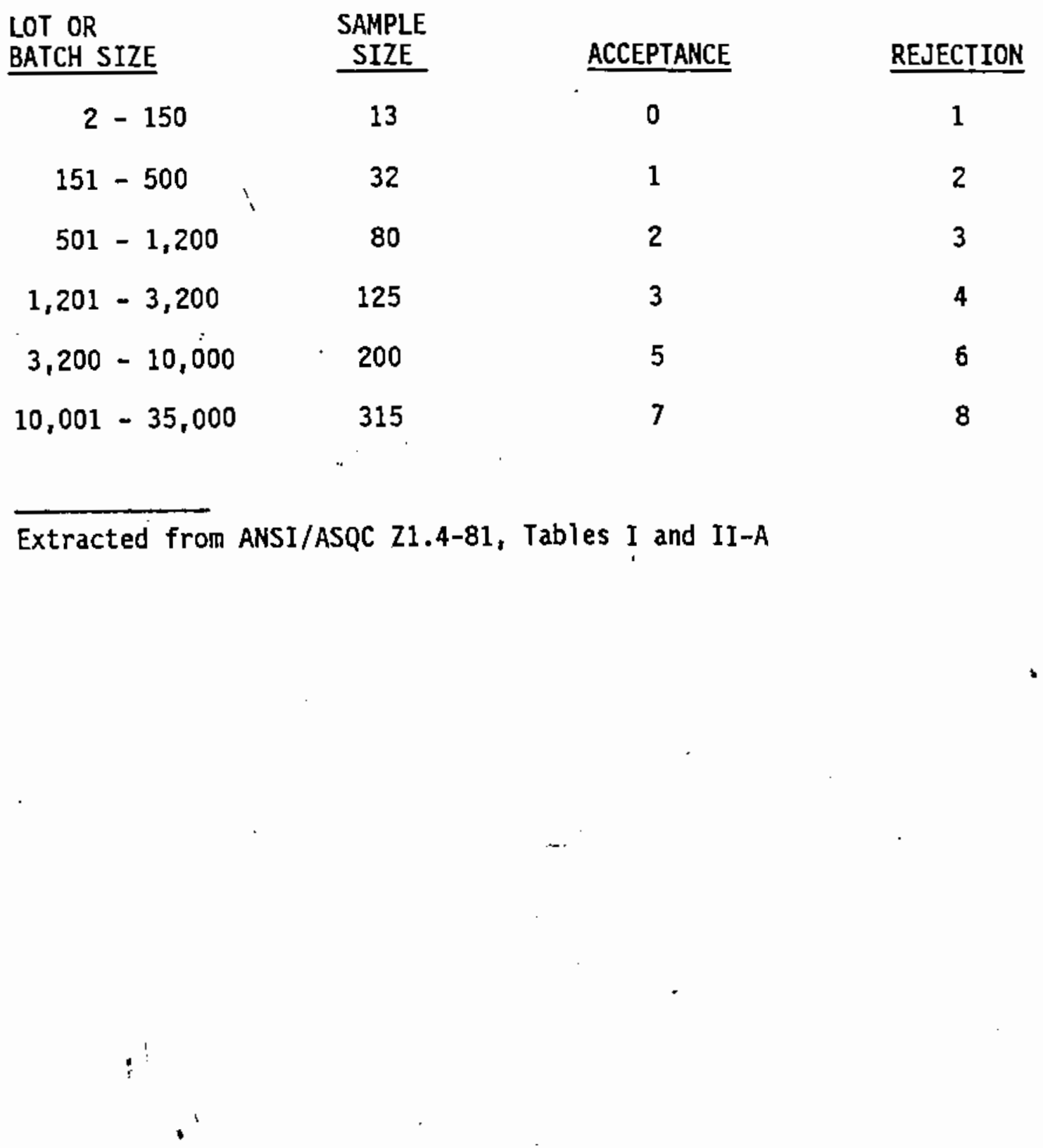

LOT OR

BATCH SIZE

$2-150$

$151-500$

$501-1,200$

$1,201-3,200$

$3,200-10,000$

$10,001-35,000$

\section{SAMPLE}

SIZE

13

125

200

315
ACCEPTANCE

0

7
REJECTION

1

8

Extracted from ANSI/ASQC Z1.4-81, Tables I and II-A 


\title{
PROCEDURE P-3: STATISTICAL ANALYSIS OF DATA
}

\author{
Hazardous Material Monitoring Project \\ RCRA Compliance Ground-Water Monitoring Project
}

\subsection{OBJECTIVES}

Data collected for the above projects will be routinely summarized for reporting to the sponsors and regulators, and less frequently analyzed for the assessment of the ground-water regime. Procedures for accomplishing the summaries that are routinely applied to these data will be included in this procedure as they are developed.

It is also anticipated that other statistical techniques, such as time series analysis, factor analysis, regression analysis, geostatistics, and other techniques that are appropriate to analyzing the data will be used in the assessment of the ground-water system. However, these will not be applied on a routine basis and will not appear in this procedure. Application of these non-routine procedures wi 71 , however, be expected to follow the necessary documentation requirements given in $Q A P 1$ an $E D-41$, Section 3 - Design Contro1 and Method Review, Addition 6 - System Maintained Software and Support Software.

At this time the routine procedures applied to the data are summaries described in "RCRA Ground-Water Monitoring Technical Enforcement Guidance Document (Draft) ", 1985, Envi ronmental Protection Agency. These summaries list and summarize the data with simple descriptive statistics calculated for different combinations of the data and plot the data for visual review. The steps needed to accomplish this are outlined below in 3.0 Generating Summaries (Simple EPA Summaries).

Other statistical procedures that are planned to be applied on a routine basis in the future are trend tests to identify statistically significant increases in contamination, quality control charts to look for off-nomal levels of contamination or outliers, and hypothes is tests for statistical significance of possible contamination. Examples of these methods and others can be found in "Ground-Water Monitoring Plans and Statistical Procedures to Detect Leaking at Hazardous Waste Facilities", 1986, PNL-5754. However, all of these procedures depend on having 15 to 20 data points before they can be applied. The procedures for applying these methods will be developed as the quantity of data allows and will be included in this procedure as they become available.

\begin{tabular}{|c|c|c|c|}
\hline \multicolumn{2}{|c|}{ 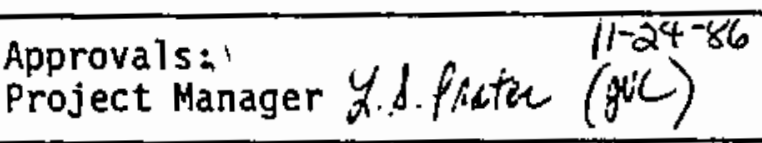 } & \multicolumn{2}{|c|}{ QA Rep OR Mhle $11-14-81$} \\
\hline $\begin{array}{l}\text { Procedure No: } \\
\text { P-3 }\end{array}$ & $\begin{array}{l}\text { Revision No: } \\
0\end{array}$ & $\begin{array}{l}\text { Date Issued: } \\
11-24-86\end{array}$ & Page 1 of 3 \\
\hline
\end{tabular}


Procedure for Statistical Analysis of Data, P-3

\subsection{REQUIREMENTS}

The Project is required under 40 CFR 264 to determine the rate and extent of contamination and determine concentrations of hazardous waste constituents in ground-water. In fulfillment of this requirement, "RCRA Ground-Water Monitoring Technical Enforcement Guidance Document (Draft)*, 1985, Environmental Protection Agency, describes certain basic summaries of the data that the regulators would like available to them when they are reviewing the status of any ground-water monitoring network. The following section defines the procedures that are used to generate these suggested sunuraries.

The data used as input to the programs discussed in this procedure are available in PNL's Hanford Ground-Water Database (HGWDB), and are entered into the database by procedures describẹ in Procedure P-2, Data Handiing and Verification.

All of the programs discussed in this procedure are run on PNL's WVAX, which is a VAX-11/780. The latest version of the operating system is VMS 4.4. System supported software packages used for this section are: VAX/DATATRIEVE, Version 3.4, used to extract data from the HGWDB and prepare a card image file for input to sumnarization programs: and Statistical Analysis System (SAS), Version 5.03 , used to read in the data, organize it, sumarize $i t$, and generate tables and graphics.

The programs and data files for each separate project covered by this procedure are maintained on individual project accounts on the WVAX. Within each project account there is a file called NOTE.BOOK that contains the latest instructions for generating the tables and plot for that project.

\subsection{GENERATING SUMMARIES (SIMPLE EPA SUMMARIES)}

The following steps must be followed to generate the simple EPA summaries.

1. Login to a personal account on the WVAX computer and SET PROJECT to the appropriate project account (Note: you must have permission to access the project account). Review the NOTE.BOOK file in the project account to ensure that the most recent report generation instructions are being followed for that project. Each project will have the same basic programs except for header information, lists of well names, definition of site-specific constituents, and other possible minor differences.

2. Retrieve the necessary raw data from the HGWOB using the appropriate DATATRIEVE command files by entering "DTR efilename" at a VMS prompt or by submitting a batch job with a similar statement in $i t$. As an example the following DATATRIEVE command files are used to retrieve raw data for the 183-H Solar Evaporation Basins:

'ANA183H.DTR for chemical constituent data (ANADAT relation) HYD183H.DTR for water level data (HYODAT relation) TMP183H.DTR for temperature data (TMPDAT relation)

\begin{tabular}{|c|c|c|l|}
\hline $\begin{array}{c}\text { Procedure No: } \\
\text { P-3 }\end{array}$ & $\begin{array}{c}\text { Revision No: } \\
0\end{array}$ & $\begin{array}{c}\text { Date Issued: } \\
11-24-86\end{array}$ & Page 2 of 3 \\
\hline
\end{tabular}


Procedure for Statistical Analysis of Data, P-3

3. Read the retrieved raw data into SAS and give simple listings by using the appropriate SAS command files by entering "SAS filename" at a VMS prompt or by submitting a batch Job with a similar statement in it. As an example the following SAS command files are used for the 183-H Solar Evaporation Basins:

ANA183H.SAS for the chemical constituent data

HYD183H.SAS for the water level data

TMP183H.SAS for the temperature data

Review of the output from the program for chemical constituent data is necessary as it contains information that may be needed for updating two programs referenced in the next step: LISTS.SAS and PERIODS.SAS

4. Prepare chemical constituent data for input to EPA summary programs by using the following programs. The three following SAS command files are not run themselves but are read into other SAS command files referenced below. They contain defining information that is not included in the HGWDB but is used for organizing the data. HOBOKEN.SAS is in its final form, but LISTS.SAS will have to be updated whenever a constituent subset changes, while PERIODS.SAS will have to be updated each sample period. HOBOKEN.SAS - Defines data analyzed by UST/Hoboken LISTS.SAS - Defines constituent subsets for the Short List PERIODS.SAS - Defines the sample periods by dates of sampling

Once LISTS.SAS and PERIODS.SAS are appropriately updated, run the following program by entering "SAS ABOVEDL" at the VMS prompt or submitting a batch job with a similar statement in it. This program separates the data into two SAS datasets for further processing. One, called BELOWDL, is the most basic summary of the data and is used as input to TABLE60.SAS in the next section. The other dataset is called ABOVEDL. It contains al] the raw data for each constituent that had at least one value reported as being above the detection limit and is used as input to all the other simple summary programs.

\section{ABOVEDL.SAS - Separates ANA183H into BELOWDL and ABOVEDL}

5. The following programs actually produce the output that is reported to the sponsors and regulators. Each program is run by entering "SAS filename" at the VMS prompt or submitting a batch job with a similar statement in it.

TABLE60.SAS - Summary for a11 constituents (total, above DL)

TABLE61.SAS - Raw data listing per constituent > DL

TABLE62.SAS - Simple statistical summaries by Constituent

TABLE63.SAS - Simple statistical summaries by constituent, well

TABLE64.SAS - Simple summaries by Constituent, Wel1, Sample Date

" TABLE65.SAS - Simple summary by ranking descriptive statistics PLOT183H.SAS - Does time plots of constituents

\begin{tabular}{|c|c|c|c|}
\hline $\begin{array}{c}\text { Procedure No: } \\
\text { P-3 }\end{array}$ & $\begin{array}{c}\text { Revision No: } \\
0\end{array}$ & $\begin{array}{c}\text { Date Issued: } \\
11-24-86\end{array}$ & Page 3 of 3 \\
\hline
\end{tabular}


Change Control Procedure, P-4 for RCRA Complfance/Hazardous Materials Monitoring

\subsection{GENERAL}

This procedure details the methods for initiating, processing, implementing and recording changes to the functional characteristics of approved documents affecting design, fabrication, construction, installation or testing.

All changes shall be traceable from need identification through completion of physical construction and incorporation of the changes into the permanent as-built construction drawings and specifications and into the technical and/or schedule control documentation.

\subsection{REQUIREMENTS AND FORMAT}

2.1 Authorized changes to the approved documents listed in Paragraph 1.0 above, shall be documented on a Design/Field Change (DFC) form

(Attachment 1). The DFC form is to be used only for changes occurring during the construction phase.

\subsection{PROCESSING DESIGN/FIELD CHANGES}

3.1 DFCs may be originated by either the PNL Geologist or the Project Manager. Al1 DFCs shall be typed or completed in black indelible ink (including all approval signatures).

3.2 Upon determination of the requi rement for document revision, the person(s) requesting the change shall contact the Project Manager, or his designate, to explain and justify the change request. Upon concurrence of the Project Manager that a revision is required, a DFC shall be initiated. The DFC requester shall be given the responsibility of authoring the DFC.

3.3 The DFC author shall list on the form a DFC number obtained from the Project DFC Log maintained by the field record custodian. The project number and title shall be written on the form.

\begin{tabular}{|c|c|c|c|c|}
\hline \multicolumn{2}{|c|}{$\begin{array}{l}\text { Prepared by: } \\
\text { R.h. Oabetg (ASp pgr) } \\
11-24-86\end{array}$} & \multicolumn{2}{|c|}{$\begin{array}{c}\text { Approvals: } \quad 11-24 f 0 \\
4 . J \cdot \text { Path (gic) } \\
\text { Project Manager }\end{array}$} & $\begin{array}{c}\text { QR Cahl } 11-14-8 x \\
\text { QA Rep }\end{array}$ \\
\hline $\begin{array}{l}\text { Procedure No: } \\
\qquad-4\end{array}$ & $\begin{array}{r}\text { Revis } \\
\quad 1\end{array}$ & & $\begin{array}{l}\text { Date Issued } \\
11-24-86\end{array}$ & Page 1 of 4 \\
\hline
\end{tabular}


Change Control Procedure, P-4

3.4 The form shall contain a listing of all documents affected by

. implementation of the DFC, including drawings, Acceptance Test

- Procedures, specifications, Nonconformance Reports, and other DFCs.

3.5 The DFC author shall enter a description of the change on the form. Sufficient detail to allow implementation of the change must be contained in the description. Required sketches must be attached, with the DFC number and a place for review and approval contained in each sketch page. All pages (inc)uding attachment) shall be numbered sequentially.

3.6 The DFC author shall enter a clear and concise justification for the DFC.

3.7 The DFC author reviews the change control form for completeness, signs the DFC and enters phone number, organization, and date.

3.8 The DFC author enters under "Remarks" any data or references which, while not directly pertaining to the particular DFC, may make the DFC more clear or more easily evaluated.

3.9 The DFC author shall obtain the following signatures (as a minimum):

- Project Manager (if not originator)

- Quality Engineer

- Senior Technical Reviewer

other organizations may need to review/approve the DFC, and it shail be the responsibility of the DFC author and/or the Project Manager to assure that other required signatures are obtained.

3.10 Approval of the Project Manager or a senior technical reviewer may be obtalned by telephone/radio. In such cases, the person obtaining the approval shall document the approval on the DFC and initial and date each approval received. The DFC author shall arrange to obtain the signatures as soon as possible on the first normal day shift.

3.11 A staff member designated by the Project Manager shall maintain the DFC Log for all DFCs associated with project-directed work. This log shall record a 1 ist of DFCs sequentially numbered by Project. Numbers are assigned upon request.

1

\begin{tabular}{|c|c|c|c|}
\hline $\begin{array}{c}\text { Procedure No: } \\
\mathrm{P}-4\end{array}$ & $\begin{array}{c}\text { Revision No: } \\
1\end{array}$ & $\begin{array}{c}\text { Date Issued: } \\
11-24-86\end{array}$ & Page 2 of 4 \\
\hline
\end{tabular}


Change Control Procedure, P-4

The staff member designated to maintain the DFC Log reviews approved DFCs for completeness (returning incomplete DFCs to the Project Manager for completion of the incomplete portions); verifies the distribution shown on the DFC is complete and up to date; fills in the block with the latest approval date; and distributes the DFC, retaining the original in the appropriate Project file and logging the final approval date in the DFC Log.

3.12 DFC numbers which are assigned but not approved and issued shall be voided by Project files upon notification from the requesting individual or the Project Manager. The DFC Log. shall be updated to show the voiding of the numbers. Numbers shall not be reassigned. Voided DFCs shail be so noted in the DFC Log.

\subsection{REVISIONS TO DESIGN/FIELD CHANGES}

Prior to final approval of a DFC, revisions may be made through use of a single line strike out on the original (black indelible ink only). New information shall be entered, and the originator of the revision shall initial and date the change. If the change is of a technical nature, all approval signatures obtained prior to the revision must be initialed and dated by the approver(s) subsequent to the change. Changes having no impact on the technical aspects of the DFC shall be initialed and dated by the author of the change. Revisions to approved DFCs which have already been issued require initiation of a new DFC (with a new number).

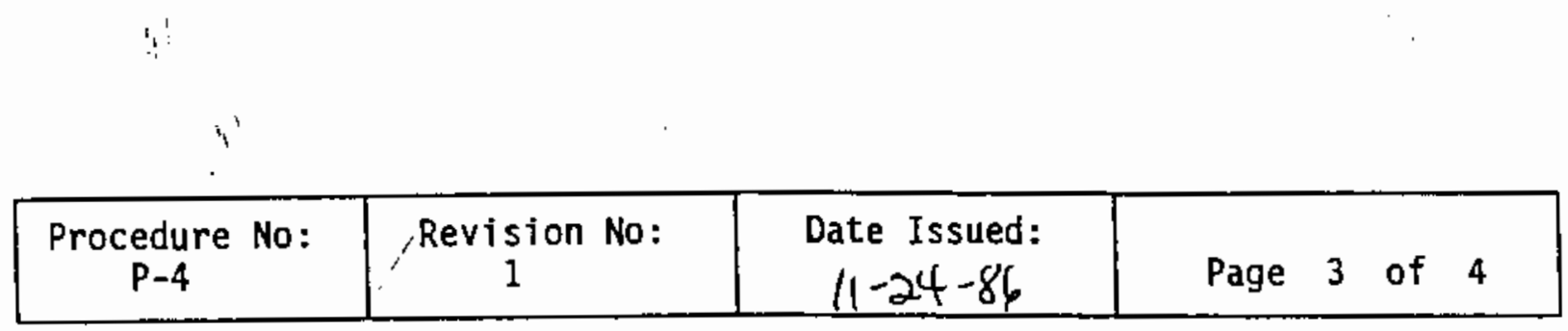




\section{ATTACHMENT 1}

\begin{tabular}{|c|c|c|c|}
\hline \multicolumn{3}{|c|}{ DESIGN/FIELD CHANGE } & \multirow{2}{*}{$\begin{array}{l}\text { DFC No. } \\
\text { Final Approval Date }\end{array}$} \\
\hline Task No. & \multicolumn{2}{|c|}{ Project Title } & \\
\hline \multicolumn{4}{|c|}{ Documents Affected } \\
\hline \multicolumn{4}{|c|}{$\vdots$} \\
\hline \multicolumn{3}{|c|}{ Description of Change } & \multirow[t]{2}{*}{ Distribution } \\
\hline \multicolumn{3}{|c|}{ Justification } & \\
\hline \multicolumn{4}{|l|}{ Remarks } \\
\hline Initiator/Author & Phone & Organization(s) & Date \\
\hline \multicolumn{4}{|c|}{ Approvals } \\
\hline \multicolumn{2}{|c|}{ Project Mạnager } & \multicolumn{2}{|l|}{ QA } \\
\hline \multicolumn{2}{|c|}{$'$} & & \\
\hline
\end{tabular}


Procedure for Water-Level Measurements for the 100- $\mathrm{H}$ Area Water-Level Network, P-5 for RCRA Compliance/Hazardous Materials Monitoring

Introduction Water-level measurements are taken approximately twice a month. All wells in the current network are measured on the same day. These measurements are taken as depth-to-water from the top of the well casing. They must be subtracted from the surveyed elevation of the casing to obtain the elevation of the water table. The water-table elevations obtained for all wells in the network can be used to produce a contour map showing the ground-water surface on the day that the measurements were made. These contour maps can be used to help characterize the ground-water flow system and to ensure that the sampling network is adequate.

Equipment The following equipment will be needed:

- graduated steel measuring tape with attached weight

- blue carpenter's chalk

- 100-H Area Water-Level Network field notebook

Procedure If the steel tape has a zero reading point, chalk the 1-ft section of tape below it. If the steel tape has no zero reading point and has divisions in hundredths of a foot all along it, then chalk several feet of the botton portion of the tape.

Estimate the approximate depth to water by using the most recent of previous measurements.

Lower the steel tape into the well to the estimated water level. Read the value of the "hold point" from the tape while holding it even with the well's measuring point (marked with paint) on the top of the casing.

Remove the steel tape and check the wetted portion at the end of the tape.

NOTE: If the chalked portion is not wet repeat the procedure using a greater hold point. If the chalked portion is completely wet repeat the procedure using a lesser hold point.

If the tape has the 1-ft section of divisions then add the unwetted length of the chalked portion of the tape to the hold point value to obtain the depth-to-water measurement. If the

\begin{tabular}{|c|c|c|c|}
\hline $\begin{array}{l}\text { Approvals: } \\
\text { Project Manage }\end{array}$ & sioh S Prater & QAD Rep. & $h / 12 / i 7 / 86$ \\
\hline $\begin{array}{l}\text { Procedure No: } \\
\mathrm{P} \rightarrow 5\end{array}$ & Revision No: & $\begin{array}{c}\text { Date Issued: } \\
1-5-87\end{array}$ & Page 1 of 2 \\
\hline
\end{tabular}


Procedure for Water-Level Measurements for the 100-H Area Water-Level Network, P-5

tape has divisions all along it's length then subtract the wetted length of the chalked portion of the tape from the hold point value to obtain the depth-to-water measurement.

Repeat the procedure until two steel tape measurements agree within at least $\pm 0.05 \mathrm{ft}$.

Record the date, well number, depth-to-water measurements, time of measurements, steel tape number, and the initials of the person taking the measurements in the 100-H Area WaterLevel Network field notebook. If you must subtract the wetted length to obtain the depth to water measurement then record the hold point and the length of the wet portion as well as the depth-to-water measurements.

Procedure No. / Revision No. P-5 
REVIEW OF QUALITY CONTROL PROCEDURES FOR COLLECTION OF SAMPLES

Authors: Roseanne L. Aaberg/Khris 8. Olsen

Date of Transmittal to Reviewers: June 17, 1987

Revile ewers:
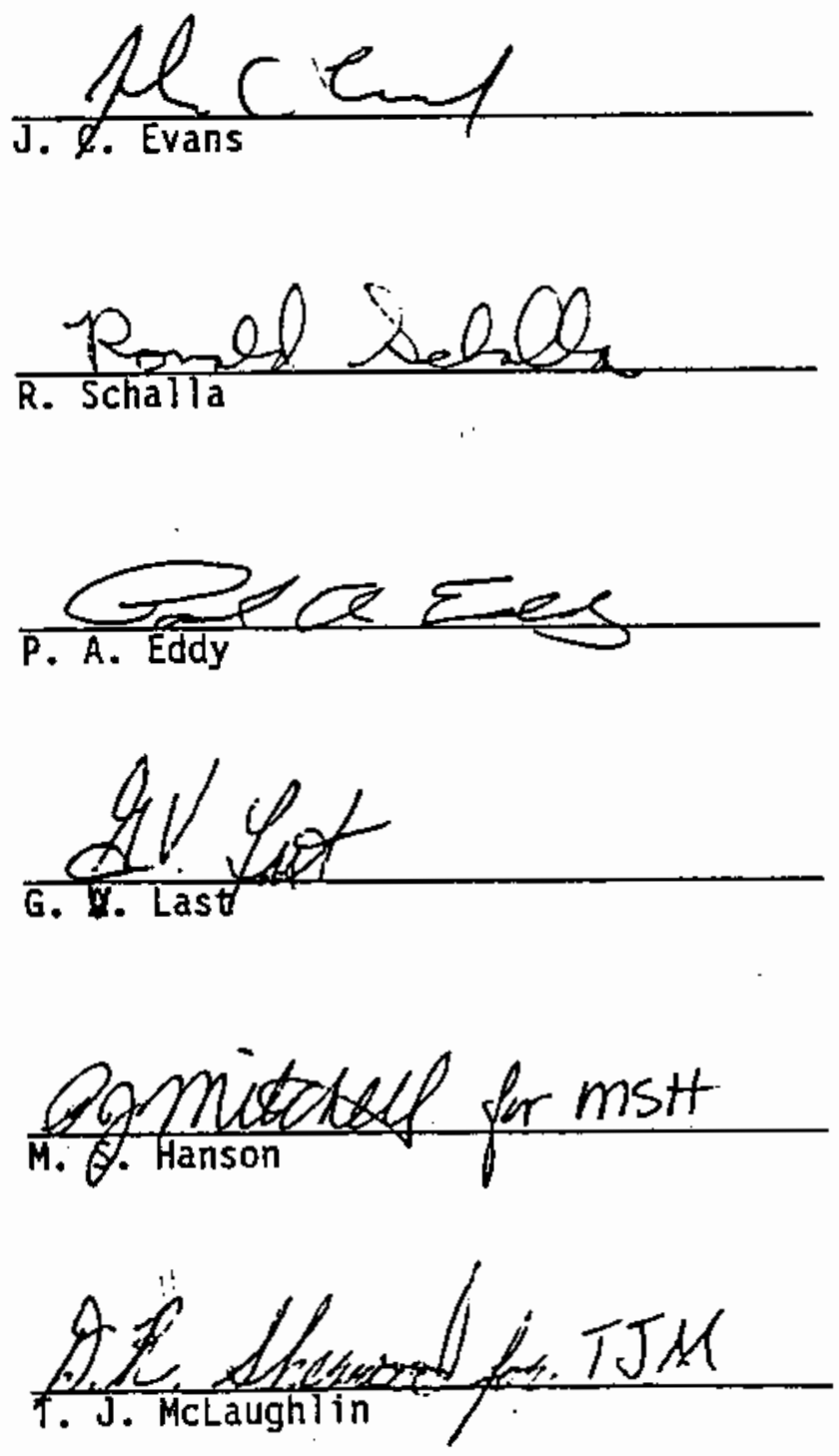
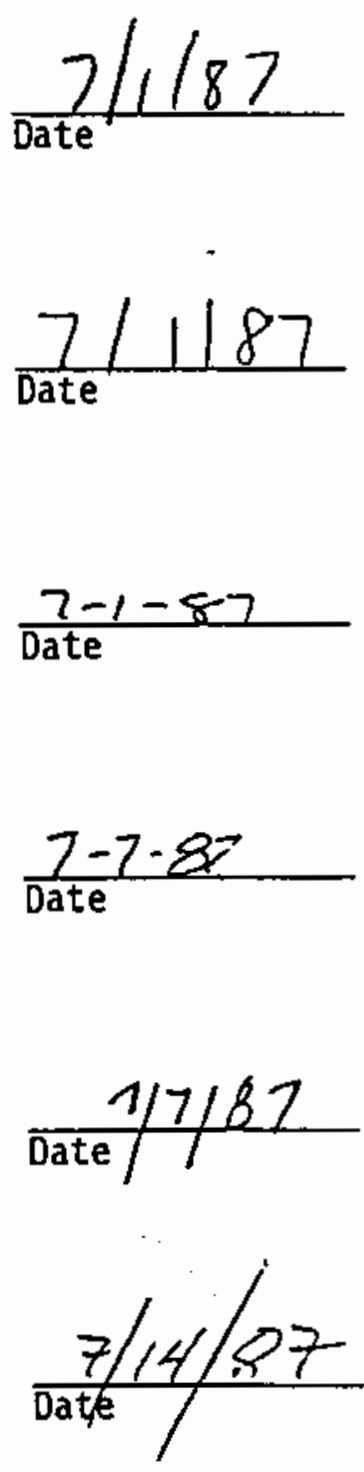

E. 48 
QUALITY CONTROL PROCEDURES FOR COLLECTION OF SAMPLES

P-7 for RCRA, CERCLA, SARA and other Compliance/Hazardous Materials Monitoring

\subsection{OB̉JECTIVE}

This procedure provides instruction for collecting and handling quality control samples from monitoring wells on the Hanford site. These quality control samples include transport and transfer blanks, intralaboratory and interlaboratory duplicate samples, and biind standards.

Transport and transfer blanks are used to check for contanination arising from the transportation or collection of samples for volatile organic analysis (VOA). Intralaboratory duplicate samples are used to determine sampling variability. Interlaboratory samples are used to determine,laboratory variability. Blind standards are used to assess the accuracy of laboratory analysis.

\subsection{APPLICABILITY.}

This procedure applies to blanks, splits, duplicates and blind standard samples collected for quality control purposes. These procedures shall be performed according to the schedule presented below for all PNL managed CERCLA, RCRA, SARA, and Hanford site groundwater monitoring programs.

One transfer and one transport blank shall be processed per 20 VOA samples, or one in each sampling area during each sample period which ever is greater ${ }^{1}$. The well selected for transfer and transport blanks shall be determined by the sample collection task leader(s). Wells designated for transfer and transport blanks shall be rotated.

Duplicate samples for U. S. Testing (UST) and interlaboratory comparison will be taken twice per quarter, or when otherwise deemed necessary by the quality control and statistical analysis task leader(s). Duplicate samples for intralaboratory and Interlaboratory comparison will be taken from the same well as the transfer and transport blank and on the same schedule. The schedule shall be determined by the quality control task leader(s) or project manager.

Blind standard samples shall be prepared quarterly to monitor the performance of the analytical laboratory. The schedule shall be determined by the quality control task leader.

1. United, States Environmental Protection Agency, November, 1986. Test Methods for Evaluating Solid Waste, Volume II.

\begin{tabular}{|c|c|c|c|}
\hline $\begin{array}{c}\text { Procedure No: } \\
P-7\end{array}$ & $\begin{array}{c}\text { Revision No: } \\
0\end{array}$ & Date Issued: & Page 1 of 3 \\
\hline
\end{tabular}


Author: Don Glover/Khris B. Olsen

Date of Transmittal to Reviewers: November 10, 1987

Reviewers:

J.d. Evans, Project Manager Hazardous Material Monitoring

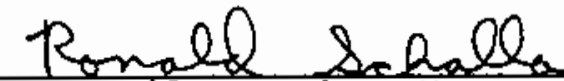

R. Schalla, Project Manager

300 Area Groundwater Monitoring

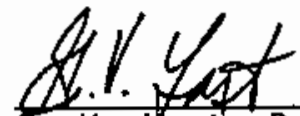

G. V. Last, Project Manager

200 Area Groundwater Monitoring

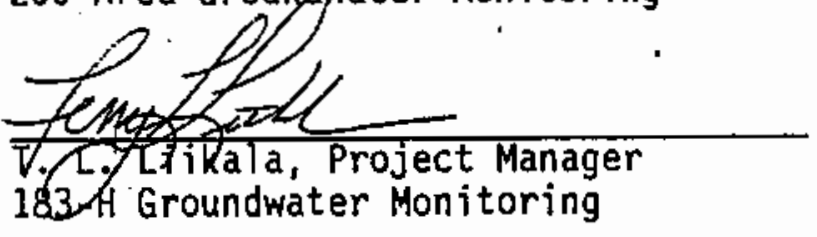

Ogmituel

P. J. Mitchel1, Program Manager

SubSurface Monitoring and Compliance Analysis
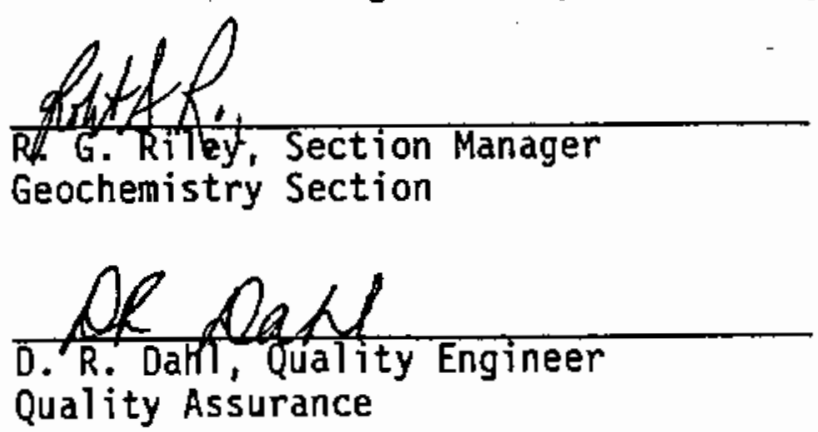

R.nn. Lmice

R. M. Smith, Task Group Leader.

Hydrology Section.'

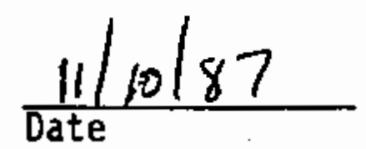

$\frac{11-10-87}{\text { Date }}$

$1 / \cdot 12-87$

Date

$\frac{11-10-87}{\text { Date }}$

$\frac{11-10-87}{\text { Date }}$ 
Sample Collection Using Bladder Pump
If the well has a dedicated bladder pump, turn on the air compressor and use the procedure described below. If the well does not have a bladder pump, use the procedures given for the Teflon bailer to collect the remaining samples.

The diagram of the original systen is seen in Figure 1A. The hookup of the 3017 low submergence adapter can be seen in Figure 1B. All hose ports and connections are well marked on the device. A small vacuum pump (Gast pump) will be used for suction instead of the air compressor inlet as noted in Figure $1 \mathrm{~B}$.

Operate the 3111 controller in the nomal manner with the 3017 low submergence adapter as shown in Figure 18 . Optimum refill cycle times will be somewhat shorter with the 3017 installed. Note that a minimum pressure of 25 psig has to be maintained through the controller in order for the 3017 to operate. During purging, the flow throttle on the 3111 controller, located in the lower left hand corner of the controller panel, should be turned all the way clockwise so that the fuil 100 psig available from the compressor is delivered to the pump.

Five to fifteen pumping cycles are required to purge the air from the bladder pump and tubing. Full water flow from the sample supply tube should then begin. After water flows from the outlet tube, run the bladder pump for at least five minutes before taking samples.

To reduce the water-flow rate during sample collection, turn the throttle control on the left side of the control panel in the counterclockwise direction. To increase the flow rate, turn the throttle control clockwise.

To optimize pumping efficiency for a specific well depth, refer to the pump manufacturer's operating instructions. 
Figure 1A.

Driver/Controller Assembly with No Low Submergence Adapter
Figure $1 \mathrm{~B}$.

3111 Driver/Controller with 3017 Low Submergence Adapter

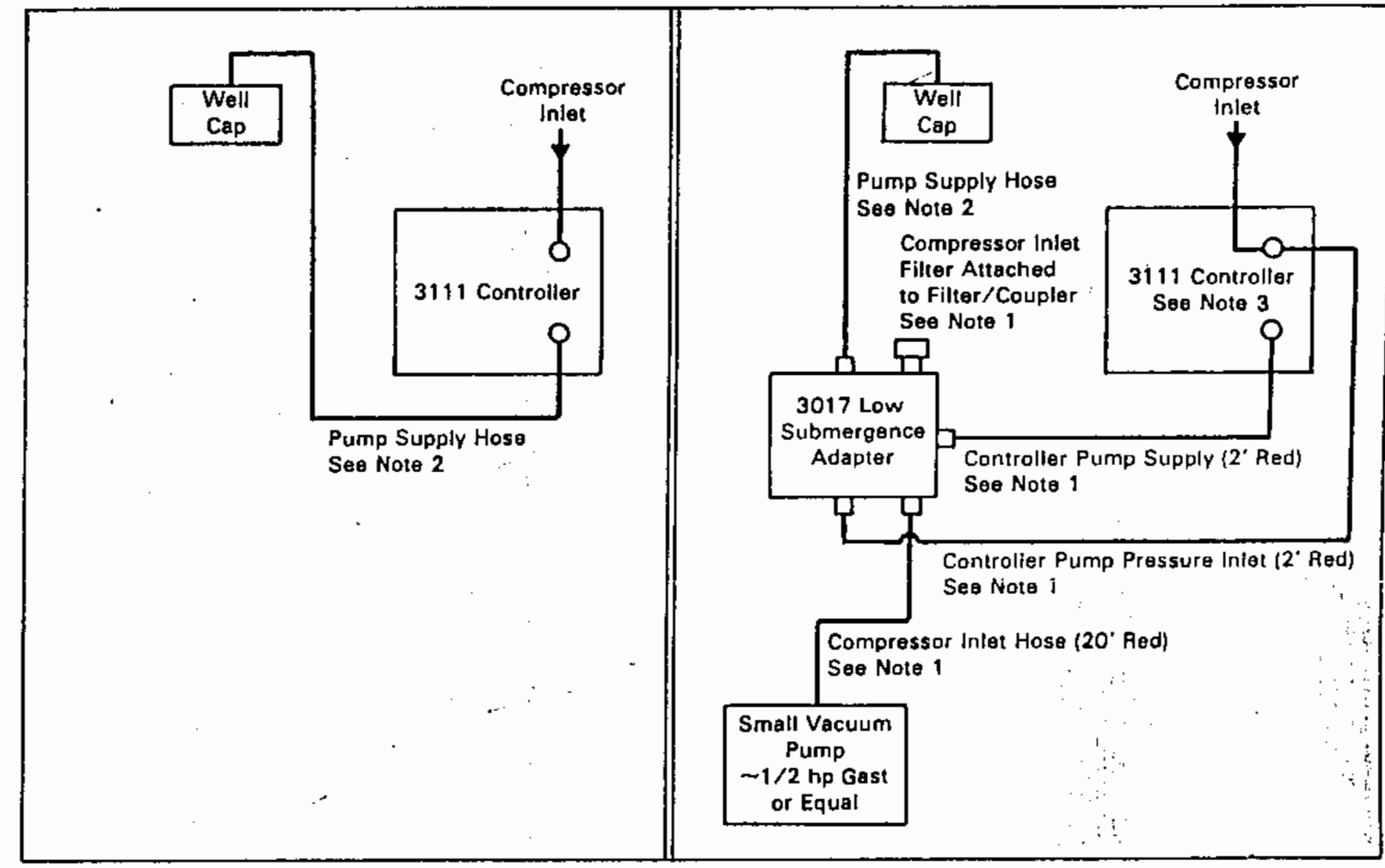

Notes:

1. Supplied with 3017 Low Submergence Adapter

2. Supplied with 3111 Compressor/Driver

3. 25 psig Minimum Required on Controller for Proper Operation 
MODIFICATION OF WELL SAMPLING PROCEDURE INTO PNL-MA-580

Author: Don Glover

Date of Transmittal to Reviewers: December II, 1987

Reviewers:

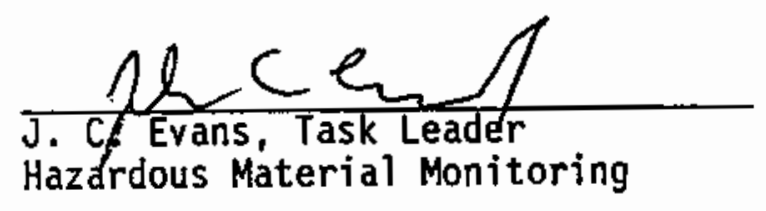

$\frac{12-11-87}{\text { Date }}$

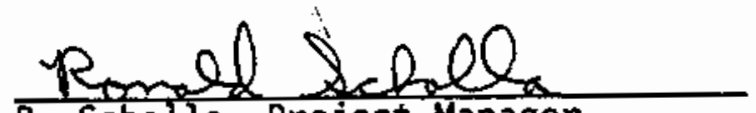

R. Schalla, Project Manager

300 Area Ground-Water Monitoring

Av Yot

G. V. Last, Project Manager

200 Area Ground-Water Monitoring

$$
\frac{12-11-87}{\text { Date }}
$$

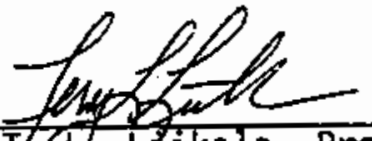

T.1. Likala, Project Manager

183-H Ground-Water Monitoring

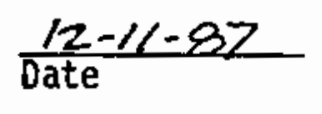

Gomitzell

P.S. Mitchell, Program Manager

Ground-Water Compliance Monitoring

$\frac{12-11-87}{\text { Date }}$

R. G. Riteyr, Section Manager Geochemistry Section

$\frac{12 / 11 / 87}{\text { Date }}$

De Daht

Q. R. Uahl, Quality Engineer

Quality Assurance

$\frac{12-14-82}{\text { Date }}$

R.m. Lnice

R. M. Smith, Technical Group Leader Hydrology Section,

$12-11-87$

Date 
PROCEDURE FOR DISPOSAL OF PURGE WATER FROM MONITORING WELLS

This procedure is intended for use in addition to existing ground-water sampling procedures in the Environmental Monitoring Procedures Manual PNL-MA-580.

Equipment Needed

- 55 gal drum liner (cut off to $24^{\prime \prime}$ height)

- 2 ea $50^{\prime} \times 3 / 4^{\prime \prime}$ garden hose

- 20 - 30 gpm sump pump

- 2 - $25^{\prime}$ extension cords

- Standard sampling equipment

- Tank truck or storage drums

Hydrostar Pump:

Place the drum liner as close to the well casing as possible. Connect the purge hose to the Hydrostar pump and place discharge end into drum liner. Connect garden hose to sump pump and extend discharge end into tank truck or storage drum. Plug the sump pump electrical cord into one of the $110-V$ outlets on the truck generator. Lower the sump pump into the drum liner and begin well purging. The sump pump will automatically keep the water level in the drum liner down during this procedure. When purging is completed, attach Teflon sampling hose and discharge water into drum liner. Fill sample bottles over drum liner so that any spillage will be collected in the drum liner. After sampling, there will be a small amount of water in the drum liner that the sump pump will not pick up. Transfer this residual water to a bucket and manually dump into the tank truck or storage drum. If disposing to storage drums, secure drum lid and band before moving to next well.

Submersible Pump

Connect the sampling manifold with garden hose to the submersible pump. Extend discharge end of garden hose into tank truck or storage drum. Place drum liner beneath valve on sampling manifold. Begin well purging. When purging is completed, open valve on sampling manifold and collect samples. After sampling, transfer residual water in drum liner to a bucket and manually dump into the tank truck or storage drum. If disposing to storage drums, secure drum lid and band before moving to next well.

Bladder Pump

Place drum liner next to well casing and discharge directly to drum liner while purging and sampling. Connect sump pump and garden hose and transfer to tank truck or storage drum as with the Hydrostar pump. After sampling, transfer residual water in drum liner to a bucket and manually dump into the tank truck or storage drum. If disposing to storage drums, secure drum lid and band before moving to next well.

Additional Requirements

Protective latex or surgical gloves will be worn during this procedure to eliminate the possibility of contaminating the samples. Care must be taken 
to assure water left in hoses and sump pump is drained into drum liner for transfer to tank truck or storage drums.

After water is disposed to the tank truck or storage drums, handling, storage, and ultimate disposal will become the responsibility of WHC waste disposal personnel. 
INCLUSION OF HYDROSTAR PUMP WELL SAMPLING PROCEDURE INTO MA-580

Author: Khris B.. 01sen

Date of Transmittal to Reviewers: June 19, 1987

Reviewers:

J. Ch Evans, Project Manager

Hazardous Material Monitoring

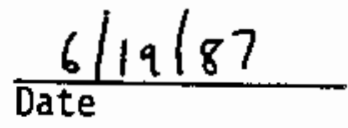

300 R. Schalla, Project Manager

$\frac{\text { Thend Q S I Schalla }}{\text { R. Schalla, Project Manager }}$

(n)

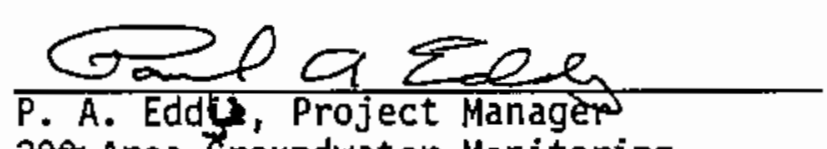

100 Ht 200 Area Groundwater Monitoring
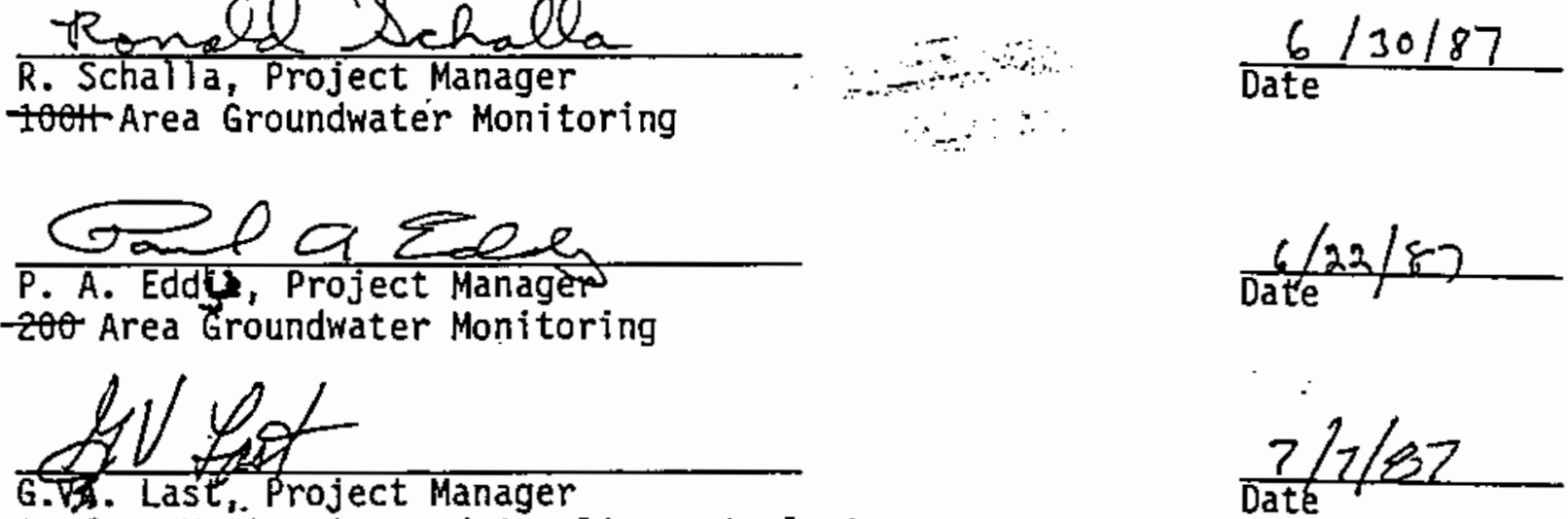

Surface Monitoring and Comptiance-Analyst's

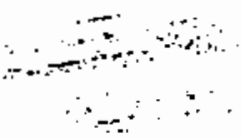

Date

200 Area Ground-water Monitoring

gamietated for mst

M.Cs. Hanson, Program Manager

Surface Monitoring and Compliance Analysis

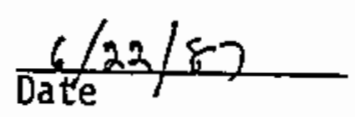

Afruatis.

J.S. Fruchter, Section Manager

Gedchemistry Section

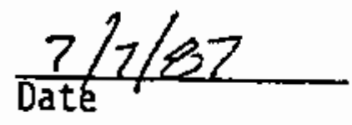

AR Daht.

D. R. Dahl, Quality Engineer

Quality Assurance

Gominaden

P. JW Mitchell, Project Manager

$\frac{7 / 7 / 81}{\text { Date }}$

Radiological Groundwater Monitoring

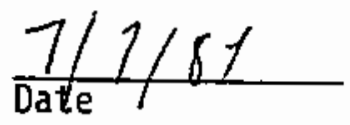

$\frac{7 / 7 / 87}{\text { Date }}$

$\frac{4|\gamma 3| 82}{\text { Date }}$ 


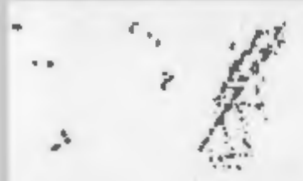

Sample Collection Using the

Hydrostar Pump
Upon arrival at the well head, immediately determine depth-to-water using the appropriate tapes, and record the determined values on the field record form.

- Wear gloves when taking samples and when handling containers, especially those with added preservative.

Attaching the Pneumatic Cylinder Assembly

1. Insert the support for the pneumatic cylinder into the column support on the well head assembly* (Figure 1).

2. Pull the cylinder rod down until it is fully extended and has stopped.

3. Align the eyelet on the top portion of the turnbolt with the clevis pin hole on the lower portion of the cylinder rod.

4. Align the hole on the cylinder support with the column support on the well head so that the turnbolt eyelet and clevis pin hole on the cylinder rod are aligned when the piston is fully extended.

5. Insert the clevis pin through one of the intersecting pairs of holes on the column support and clip a hitch pin into the holes in the small end of the clevis pin.

6. - Check the alignment on the turnbolt eyelet with the hole on the cylinder rod. The alignment must be nearly perfect, neither too high nor too low.

7. Adjust by rotating the turnbolt clockwise or counterclockwise. :

* When inserting the cylinder support into the column support on the pump assembly, at least two holes on the cylinder support must overlap with two holes on
the column support. If less than two holes overlap - use the extension supplied with the Hydrostar pneumatic cylinder. Align the pumping system in the

$\because$ same manner as described above. $\because$

\section{Operating the Pneumatic Cylinder}

1. Attach either the purging hose (large diameter) or the teflon sampling hose to the outlet on the discharge tee of the sampling pump. 

2. Attach the quick-connect on the supply hose to the unattached end of the control valve on the pneumatic cylinder. The input air pressure should not exceed 120 psi.

3. Turn air supply on to the control valve.

4. Turn on the control valve on the pneumatic cylinder. The piston will begin to operate.

5. Adjust stroke rate to no more than 60 per minute*. The stroke speed of the pneumatic cylinder can be adjusted with the control valve located on the top of the pneumatic cylinder. A stroke is defined as one downward and one upward extension.

* If the pneumatic cylinder assembly is not operating correctly, and the problems are not due to the well or the pump in the well, the well may be hand pumped as described in "Manual Operation."

\section{Sampling With Pneumatic Piston Assembly}

1. Slow down the pumping rate until the piston operates smoothly. This rate will be less than 10 strokes a minute.

2. Attach the Teflon sampling hose and purge at this rate for a minimum of two minutes.

3. Proceed with sampling all unfiltered samples according to PNL-580.

4. Attach the filter assembly and purge the filter according to directions listed in PNL-580. If too much pressure is exerted across the filter the membrane will rupture, usually resulting in a popping noise. If this happens, replace the filter and

restart the filtering procedure.

5. Dismantle the pneumatic pumping assembly as described below.

Removing the Preumatic Pumping Assembly

1. Disconnect the air supply at the pneumatic cylinder.

2. Disassemble pneumatic cylinder in reverse order of steps 1 through 7 in the section "Attaching Pneumatic Cylinder. Assembly. $\because \ldots \quad \cdots \quad \ldots \ldots . .$.

3. Replace well cap over top of well head. 


\section{Manual Operations}

1. Insert the handle support into the column support on the pump head assembly so that at least two holes on the handle support overlap with two holes on the column support (Figure 2).

2. Slide the clevis pin through one of the intersecting pairs of holes on the column support.

3. Clip the hitch pin into the hole in the small end of the clevis pin.

4. Remove the turnbolt on the top of the rod at the well head.

5. Attach the turnbolt on the end of the wire rope attached to the handle assembly onto the threaded rod at the top of the well head.

6. Lift the handle so that the flat edge of the cam nearest the shackle is approximately parallel with the ground.

7. Pull all the slack out of the wire rope.

8. Using either an adjustable or 9/16" open end wrench, tighten both nuts on the shackle until the sheath on the wire rope is compressed. Remembering to keep all the slack out of the wire rope.

\section{Manual Well Sampling}

1. Attach either the purging hose (large diameter) or the Teflon sampling hose (small diameter) to the outlet on the discharge tee of the sampling pump.

2. Begin pumping the operating handle with smooth; even strokes. For best performance, use 20 to 45 strokes per minute for purging the well. Use less than 10 strokes per minute during sampling. When the filter assembly is attached, special attention is required to prevent rapid build up of pressure across the filter. If too much pressure is exerted across the filter the membrane will rupture, usually resulting in a popping noise. If this happens, replace the filter and begin sampling for the filtered sample according to the written procedure.

3. When sampling is completed follow the direction below to disassemble the handle assembly from the well head......... 
$\because \quad \because y^{\prime}$ 


\section{Removing the Handle Assembly}

Disassemble the handle assembly in reverse order of steps 1 through 8 in the section "Manual Operation." 
Author: Khris B. .01sen

Date of Transmittal to Reviewers: June 19, 1987

Reviewers :

lle end

J.C. Evans, Project Manager

Hazardous Material Monitoring

Romald Schalla

R. Schalla, Project Manager

300 Area Groundwater Monitoring

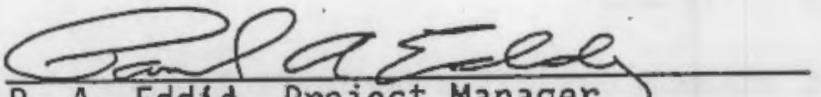

- P. A. Eddyd, Project Manager

$100 \mathrm{H}$ Area Groundwater Monitoring

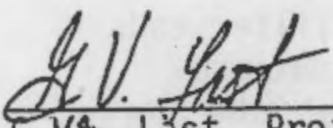

G.V.h Last, Project Manager

200 Area Groundwater Monitoring

Omitand for ms H

M.S. Hanson, Project Manager

Surface Monitoring and Compliance Analysis

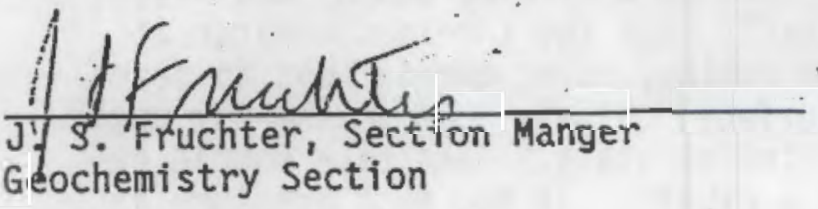

Geochemistry Section

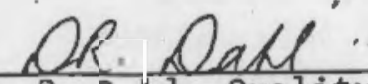

D. R. Dahl, Quality Engineer

Quality Assurance

Sgmitinell

P. Q. Mitchell, Project Manager

Radiological Groundwater Monitoring

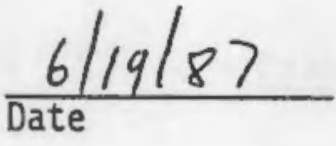

$\frac{6 / 29 / 87}{\text { Date }}$
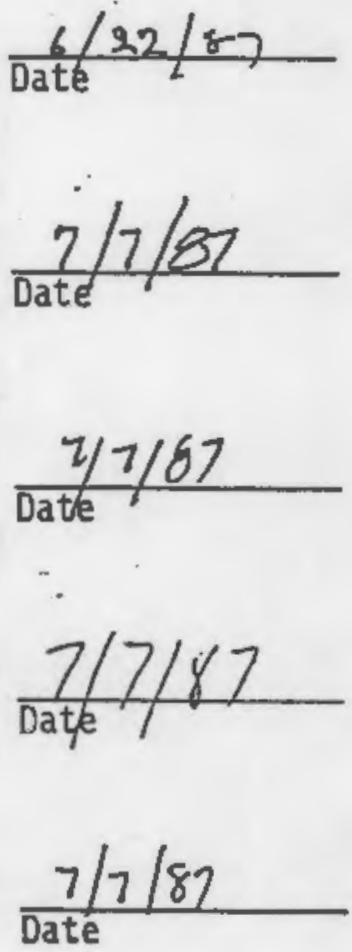

E. 63 


\subsection{IN-LINE SAMPLE FILTRATION PROCEDURE}

Introduction

Equipment

Filtration Procedure submer other
For collecting a filtered water sample, a disposable filter assembly is attached to the end of the outlet tubing of a bladder pump. Hydrostar pump, or submersible pump. One filter should be sufficient to collect the required sample volume under normal conditions. If a second filter is required, repeat the filter wash procedure described below. If the filters clog too quickly, replace the standard filter (QED Model 8100) with the high capacity model (QED Model 8000 ) and repeat the preparation procedure.

The following equipment will be needed:

$$
\begin{aligned}
& \text { QED "Sample Pro" Model } 8100 \text { or } 8000 \\
& 500-m l \text { container } \\
& \text { Tubing for the filter adapter }
\end{aligned}
$$

Follow procedure 13.2 to prepare the well for hazardous materials sampling using the bladder, Hydrostar or sible pump. Collect the filtered sample after all samples have been collected.

Bladder Pump: Set the maximum discharge pressure to 60 psi and turn off the bladder pump controller. Screw the inlet end of the filter assembly (marked inlet) into the threaded adapter, being careful not to touch filter ends to any surface. Turn on bladder pump (check maximum pressure) and filter $500 \mathrm{ml}$, as a filter wash, into the $500-\mathrm{ml}$ container. Dispose of the $500 \mathrm{ml}$ wash and collect the sample volume specified on the field record form. Turn off bladder pump, remove the filter, and return the filter to the laboratory for proper disposal.

Hydrostar Pump: Turn off air to the Hydrostar pump at the piston assembly. Screw the inlet end of the filter assembly (marked "inlet") into the threaded adapter at the end of the teflon tubing, being careful not to touch

filter ends to any surface. Slowly turn on the air until the piston just operates smoothly. This rate should be less than 10 strokes a minute. If too much pressure is exerted across the filter the membrane will rupture, usually resulting in a popping noise. If this happens replace filter and restart the filtering procedure. Filter $500 \mathrm{ml}$, as a filter wash, into the $500-\mathrm{ml}$ container. Dispose of the 500-ml wash and collect the sample volume specified on the field record form. Turn off the Hydrostar pump, remove the filter assembly, and return the filter assembly to the laboratory for proper disposal.

Submersible Pump: This pump should be used to collect a filtered sample only if a by-pass assembly is used to 
regulate the water pressure going to the filter apparatus. The by-pass must also be capable of maintaining a sufficient water flow to prevent damage to the submersible pump. Mount by-pass assembly to the well head and tefion sampling hose to by-pass assembly before turning on submersible pump. Purge well with by-pass valve completely open. To collect unfiltered samples slowly close by-pass valve until a steady flow is observed through teflon sampling hose. To collect filtered samples open by-pass valve completely. Screw the inlet end of filter assembly (marked "inlet") into the threaded adapter at the end of the tefion tubing, being careful not to touch filter ends to any surface. slowly, close the by-pass valve until a steady flow of water is observed through filter. When a steady flow is achieved, filter $500 \mathrm{ml}$, as a filter wash, into the 500-mi container. Dispose of the 500-ml wash and collect the sample volume specified on the field record form. Turn off the submersible pump, remove the filter assembly and return the filter assembly to the laboratory for proper disposal. Disconnect the by-pass apparatus from the well head. 


\subsection{REFERENCES}

McGhan, V. L., P. J. Mitchell, and R. S. Argo. 1985. Hanford Wells, PNL-5397, Pacific Northwest Laboratory, Richland, Washington.

United States Testing Company, Inc. (UST) 1986. Procedures Manual, UST-RD-PM-9-80, Rev. 3, United States Testing Company, Inc., Richland, Washington. 
APPENDIX $F$

PNL'S QUALITY ASSURANCE PLAN FOR THE HANFORD GROUND-WATER MONITORING PROJECT

AND

WESTINGHOUSE HANFORD COMPANY'S OA APPROVAL

OF PNL OA PROGRAM FOR GROUND-WATER MONITORING WELLS 
PHL-MA-70 Quality Assurance Plan No. OHE-1E, Rev. 1

QA PLAN FOR THE HANFORD GROUND-HATER MONITORING PROJECT
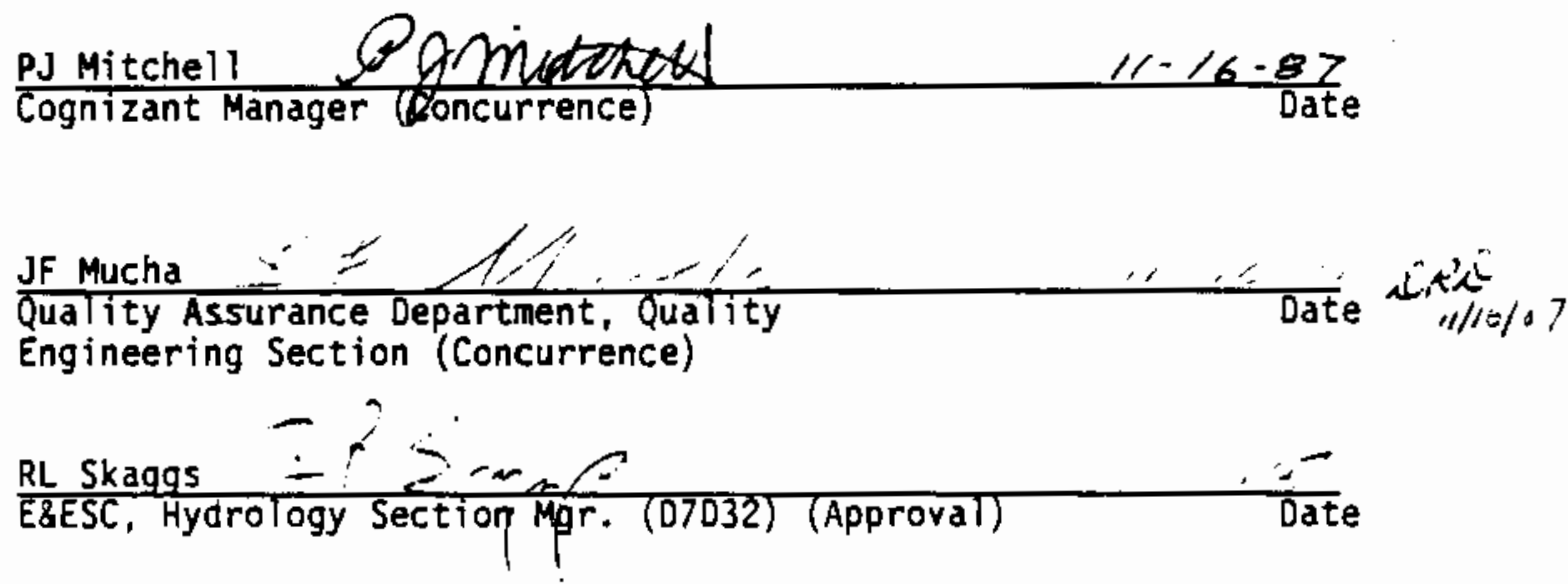
PROJECT IMPACT LEVEL II

\section{TITLE:}

QA PLAN FOR THE HANFORD GROUND-WATER MONITORING PROJECT

\section{SCOPE:}

This QA Plan, in addition to QA Plan OHE-1, applies to the PNL-managed Ground-Water Monitoring Project on the Hanford Site. Operations since 1944 at the Hanford Site have resulted in the discharge of process cooling water and other waste waters to the ground. The primary discharges have occurred within or near the 200 Areas located in the center of the site. These effluents, which have percolated to the unconfined ground-water, contain low levels of radiological and chemical contaminants. The Hanford Ground-Water Monitoring Project monitors the concentrations of these constituents in the unconfined aquifer and evaluates their impact on people and the environment. The Project is designed to meet the intent of DOE Orders 5480.1 and 5484.1, which are applicable to environmental monitoring.

The scope of work encompasses all activities that are required to collect and analyze ground-water samples for various constituents, to maintain a technically-based ground-water monitoring network, to expand the monitoring well network, to evaluate results, to estimate radiation dose-to-man attributable to constituents in the subsurface, and to publish an annual environmental monitoring report. The movement and potential redistribution of radionuclides and hazardous chemical constituents in the ground-water are investigated with sample collection and analysis, and with groundwater and geochemical modeling. A capability is maintained to respond to unusual or emergency situations.

\section{CLIENT:}

The primary $c$ lient for the Hanford Site Ground-Water Monitoring Project is the U.S. Department of Energy, Richland Operations Office.

\section{AUTHORIZING DOCUMEHT:}

11246

\section{QA REQUIREMENTS SPECIFICATION(S):}

[X] ANSI/ASME NQA-1 as delineated in PNL-MA-70

[ ] Other

This QA Plan supersedes QA PIan OHE-1E, Rev. 0, dated 10/1/87. 
QA PIan No. OHE-IE Rev. 1 Issue Date

Page 3 of 5

In addition to the requirements delineated in QA PIan OHE-1, Impact Level II activities shall comply with the applicable requirements, as appropriate for the work being performed, identified in Parts 1 and 3 of PNL-MA-70. Any activities identified as Impact Level III shall comply with the PNL Good Practices Standard, Part 2 of PNL-MA-70. If other quality-related activities are later performed, the appropriate PNL-MA-70 requirenents and procedures shall be applied to them irrespective of whether they appear herein, unless specifically excluded.

Applicable PNL-MA-70 administrative and software control procedures along with any exclusions or limitations to the procedures are identified in Attachment $A$.

Applicable technical procedures are Identified in Attachment 8 .

Any activity completed prior to the issuance of this QA Plan is exempt from the requirements delineated herein. However, these activities are subject to the requirenents delineated in the $Q A P l a n$ in $p l a c e$ at the time they were performed. Activities in progress at the time this QA Plan is issued shall be converted to meet the PML-MA-70 QA Program requirenents delineated as rapidly as practicable.

OTHER APPLICABLE OA PLANS

$\begin{array}{llll}\text { No. } & \text { Rev. } & \text { Title } & \text { Issue Date } \\ \text { OHE-1 } & 0 & \begin{array}{l}\text { QA Plan for the Hanford Site } \\ \text { Environnental Monitoring Program }\end{array} & 7 / 31 / 87\end{array}$

\section{CONCURRENCES AND APPROVAL:}

Concurrences and approvals to this QA PIan are listed on the title page.

\section{ORGANIZATION:}

Project organization is identified in Attachment C. Organizational interfaces are shown in Attachment $D$.

\section{IMPACT LEVEL:}

This QA Plan has been assigned an overall Impact Level of II. Hanford Ground-Water Monitoring Project WBS elements and corresponding Impact Levels are identified in Attachment E, WBS Impact Level Matrix. 


\section{SPECIAL CLIENT REQUIREMEMTS:}

No special client requirements have been identified.

\section{OTHER REQUIREAENTS, OIRECTION OR PLAMHING:}

MAJOR KMOWH PROCUREMENTS

Primary analytical services to be provided by U.S. Testing, Inc. are governed by subcontract B-B2472-A-I.

Washington State University will be utilized to provide an independent and objective examination of the accuracy and precision of the ground-water sampling and laboratory analyses conducted under contract B-M5964-A-E.

ACTIVITIES REQUIRIMG CERTIFIED INSPECTION PERSONMEL

None.

\section{KNOWN CONTROLLED PROCESSES}

Controlled processes performed by project personnel include environmental media sampling. Technical procedures goveming these activities are identified in Attachment 8.

PNL will supply a site geologist to oversee monitoring well network expansion and to provide geological guidance, record lithology, and collect water and sediment samples for characterization. This information, as well as well construction details, shall be recorded by the PNL geologist on a Well Completion Report form (form number A-1800-187) (Attachment F).

Controlled processes performed by others include laboratory analyses.

Qualification of the noted controlled processes shall not be required because they are based on standard, well understood methods.

\section{KNOWN SPECIAL PROCESSES}

\section{None.}

\section{DISPOSITION OF RECORDS}

Upon issuance of this QA Plan, records shall be indexed and subsequently maintained in accordance with PAP-70-1701. The retention period shall be specified as lifetime. Records will not be turned over to the client unless specifically requested. 
PNL-MA-70 QA PLAN

QA PIan No. OHE-1E Rev. I Issue Date

Page 5 of 5

PREPARATION, REVIEH, AND APPROVAL OF PURCHASE REQUISITIONS

Each item identified on a purchase requisition (PR) must have its Impact Level assessed and documented on the PR. If all items on a PR are assigned Impact Level III and no QA requirements apply, QE review and concurrence is not required.

As a minimum, all PRs shall show evidence of technical review by signature/date of reviewer in the "Technical Questions Contact" block of the PR prior to submittal for Level 3 (or above) Manager approval, and Finance and $Q E$ concurrence (if appropriate).

Subcontracts for services, including analytical services, for this project shall include appropriate quality assurance requirements for all critical activities. 
QA Plan No. OHE-IE Rev. I Attachment $A$

Page 1 of 1

\section{APPLICABLE ADMINISTRATIVE AND SOFTHARE CONTROL PROCEDURES}

The administrative procedures identified below apply to the Hanford GroundWater Monitoring Project.

PAPs are located in Volume I of PHL-MA-70 while SCPs may be found in Volume II of PNL-MA-70.

[X] PAP-70-401 PREPARATION, REVIEH, AND APPROVAL OF PURCHASE REQUISITIONS

$+*[X]$ PAP-70-801 MATERIAL IDENTIFICATION AND CONTROL (TESTING

AND EXPERIMENTATION)

[X] PAP-70-901 CONTROL OF PROCESSES

$[X]$ PAP-70-1101 TEST/ANALYSIS PLANNING, PERFORMANCE, AND EVALUATION

$+[X]$ SCP-70-312 OETERMINATION OF SOFTWARE REQUIREMENTS

$* \star X] \quad$ SCP-70-313 FINAL INTERNAL OEVELOPMENT REVIEN OF

SOFTHARE ANO DOCUMENTATION

$\star \star[X]$ SCP-70-314 SOFTWARE CONFIGURATION MANAGEMENT

$*[X]$ SCP-70-315 CONVERSION TESTING, VERIFICATION, ANO/

$* *[X] \quad S C P-70-316$ SOFTWARE APPLICATION CONTROL

$* *[X] \quad S C P-70-317$ TRANSFER OF SOFTHARE, DATA AND/OR DOCUMENTATION

$\star \star[X] \quad S C P-70-318$ CONTROL OF DATA BASES

+ See Exclusions or Limitations of Applicability below for clarification.

- Applies to identification of environmental media samples.

* Applicability determined by the requirenents of SCP-70-312.

EXCLUSIONS OR LIMITATIONS OF APPLICABILITY

In addition to those identified in QA Plan OHE-1, the following exclusions or limitations of applicability apply to the procedures identified above.

PAP-70-801: Material Identification and Control

Inventory control, identified in Sections 4.2 .1 and 4.2 .2 of PAP-70-801 shall not be required for environmental media samples. Also, the Chain of Custody Form may be used in lieu of the Material Identification Card identified in PAP-70-801.

\section{SCP-70-312: DETERMINATION OF SOFTWARE REQUIREMENTS}

SCP-70-312 through SCP-70-318 as delineated here apply to database software and data only. Computer modeling activities performed under WBS 03 have been identified as Impact Level III and therefore the GPS shall apply. 
QA Plan OHE-1E Rev. 1

Attachment $B$

Page 1 of 1

APPLICABLE TECHMICAL PROCEDURES

\begin{tabular}{|c|c|c|}
\hline Number \& Rev. & Title or Subject & $\begin{array}{l}\text { ssue Date } \\
\text { or planned) }\end{array}$ \\
\hline GWM-1 & $\begin{array}{l}\text { Ground-Water Monitoring } \\
\text { Procedures (currently } \\
\text { contained in PKL-MA-580) }\end{array}$ & TBD \\
\hline P-1 & Sample Disposal & $11 / 24 / 86$ \\
\hline $\mathrm{P}-2$ & Data Handling and Verification & $3 / 30 / 87$ \\
\hline$P-3$ & Statistical Analysis & $11 / 24 / 86$ \\
\hline$P-4$ & $\begin{array}{l}\text { Change Control Procedure for } \\
\text { RCRA Compliance/Hazardous } \\
\text { Materials Monitoring }\end{array}$ & $11 / 24 / 86$ \\
\hline$P-5$ & $\begin{array}{l}\text { Procedure for Water Level } \\
\text { Measurenents for the 100-H } \\
\text { Area Water Level Monitoring } \\
\text { Metwork for RCRA Compliance } \\
\text { Hazardous Materials Monitoring }\end{array}$ & $1 / 5 / 87$ \\
\hline P-6 & $\begin{array}{l}\text { Procedure for Collection and } \\
\text { Documentation of Drilling } \\
\text { Data for RCRA Compliance/ } \\
\text { Hazardous Materials Monitoring }\end{array}$ & $3 / 16 / 87$ \\
\hline \multirow[t]{3}{*}{$P-7$} & $\begin{array}{l}\text { Quality Control Procedures for } \\
\text { Collection of Samples for RCRA, } \\
\text { CERCLA, SARA and other Compliance/ } \\
\text { Hazardous Materials Monitoring }\end{array}$ & $7 / 23 / 87$ \\
\hline & $\begin{array}{l}\text { Hydrostar Pump Well Sampling } \\
\text { Procedure }\end{array}$ & $8 / 19 / 87$ \\
\hline & $\begin{array}{l}\text { In-Line Filtration Procedure } \\
\text { Sample Collection Using Bladder } \\
\text { Pump }\end{array}$ & $\begin{array}{l}8 / 19 / 87 \\
\text { T80 }\end{array}$ \\
\hline
\end{tabular}


00

$\$ 2913 K$
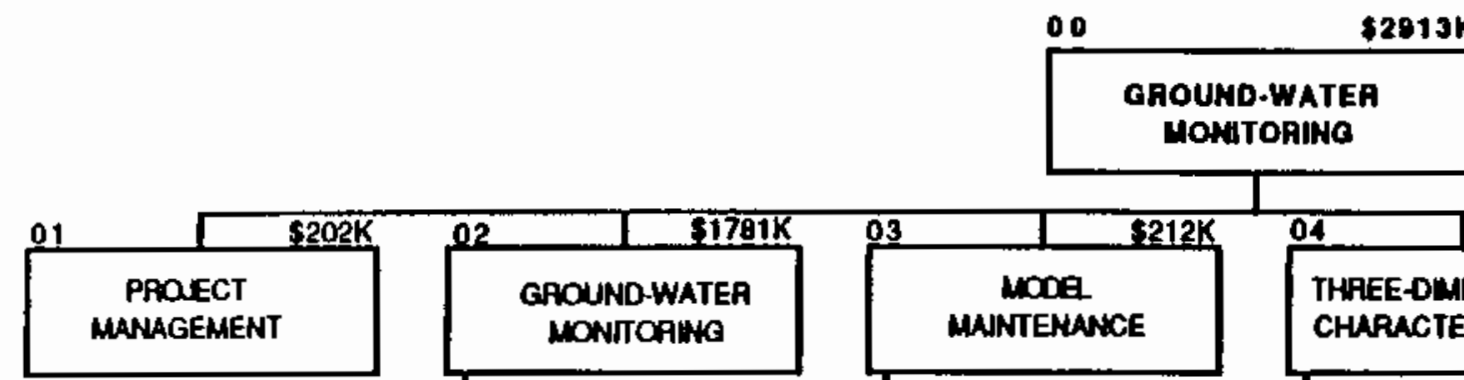

$\pi$

GROUND-WATER
MONTOAHA

0201

SAMPLING AND

ANALYSIS
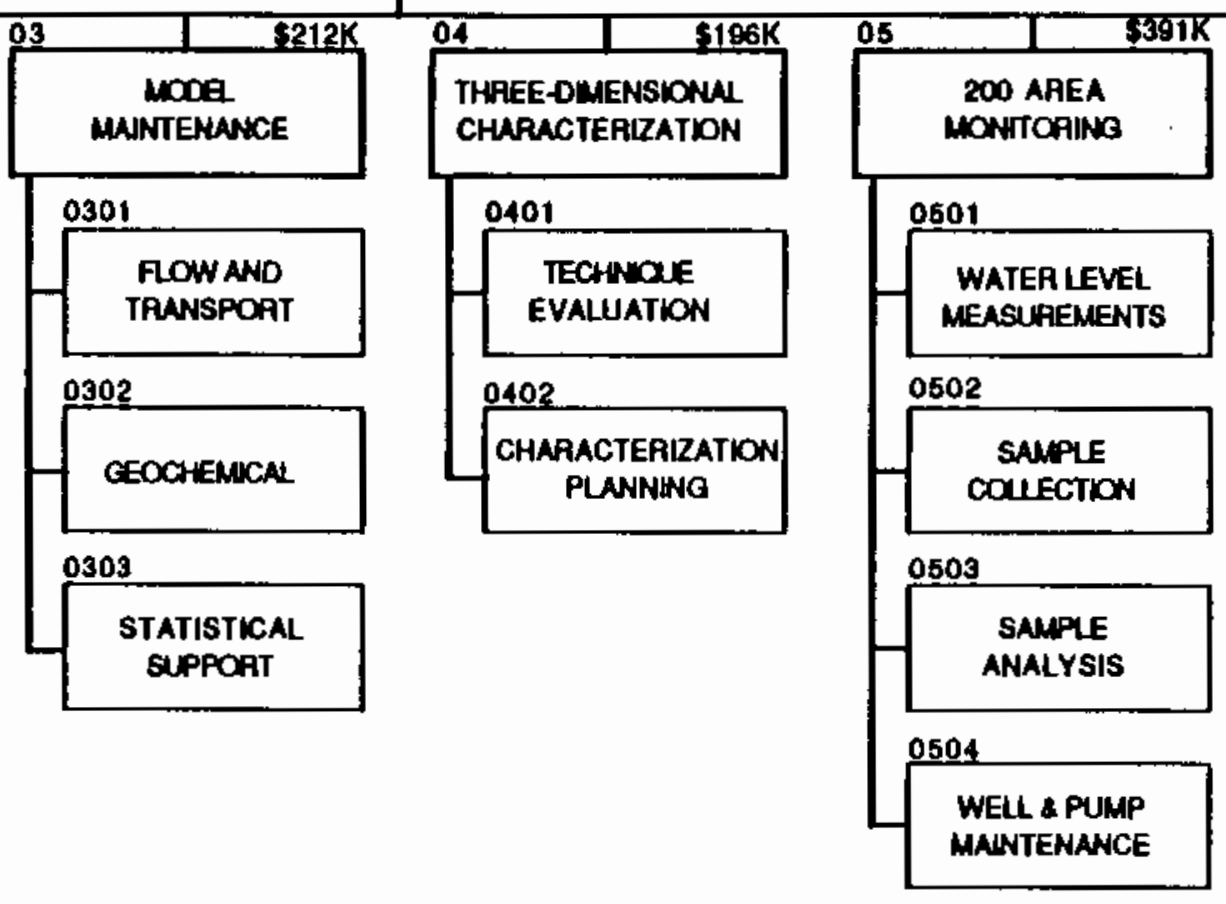
06 \$131K
MONITOANG WELL NETWORK EXPANSION

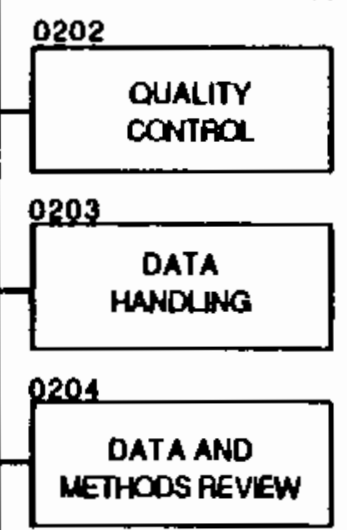

0205

WELL AND PUNP

MANTENANCE

0206

GEOPHYSICAL

CHARACTEFIZATION 


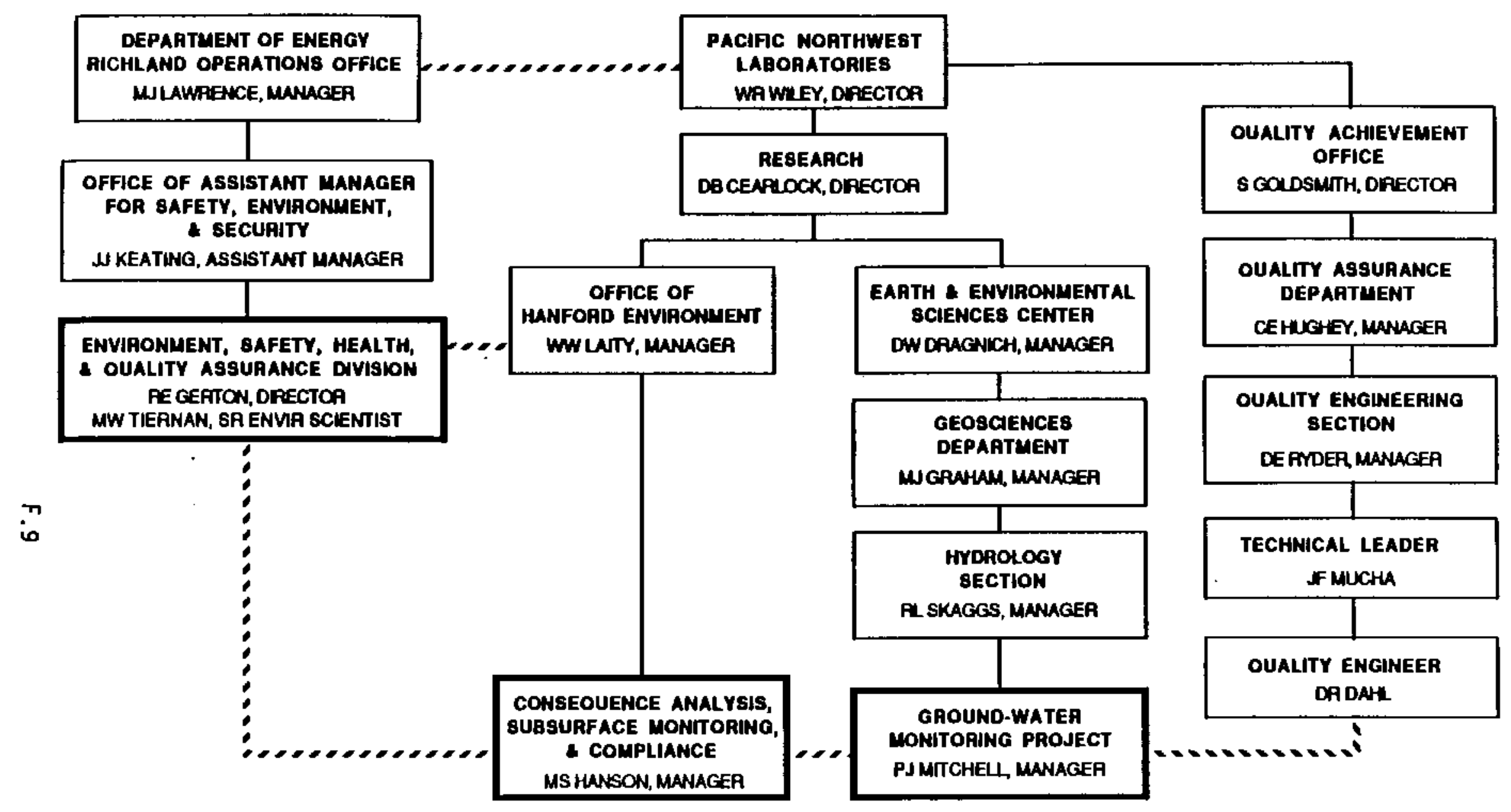

$10 \cdot 2.87$

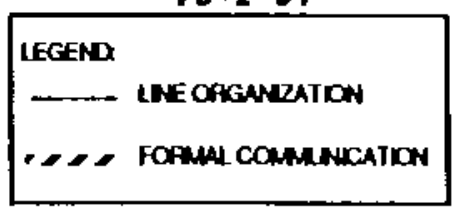


Page 1 1 ol 1

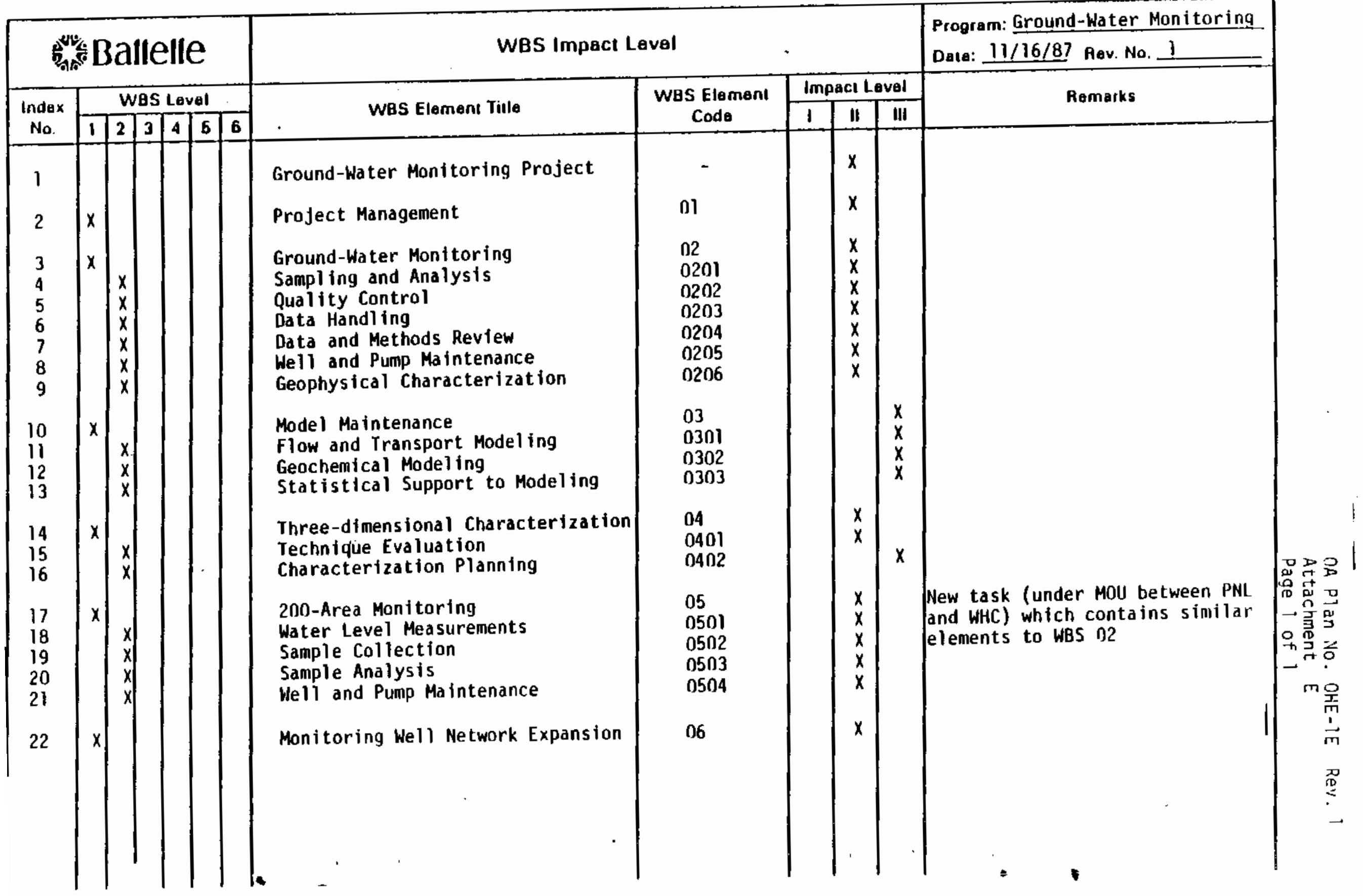




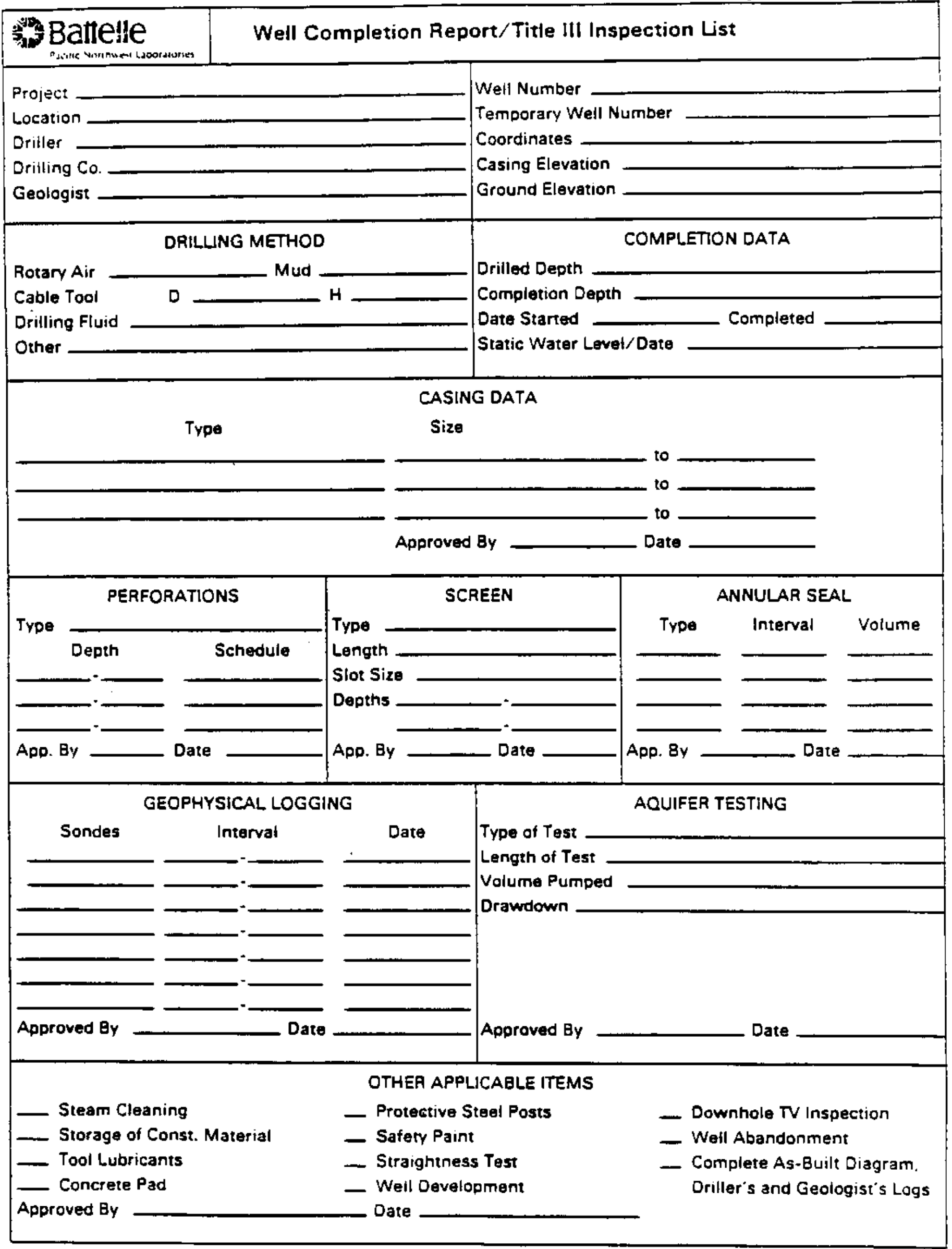




,


From: Construction Profects duality Engineering

Phone: $\quad 3-5352$ S1-54

Date: March 10, 1988

Subject: OA APPROYAL OF PNL OA PROGRAM FOR GROUNDWATER MONITORING WELLS

To:

S. R. Briggs R3-43
cc: W. H. Caplinger Nl-7l
R. B. Gelman Sl-5l
P. S. Peacock Rl-15
J. D. Schaffer S1-52 $R E C: J A P: F i T e / L B$

The iPacific Northwest Laboratories Oulity Assurance Program has been reviewed and is approved for PNL inspections of the groundwater monitoring wells. Further, the use of a qualified geologist to perform these inspections is approved based on PNL letter $\$ C C-082-M R$.S dated March 4, 1988 to S. R. Briggs from R. M) Smith.

A. Feltier

Manager 
Pacific Northwest Laboratories P.O. 80x 999

Richland, Washinglon U.S.A. 99352

Telephone ( 509 )

376-5831

Telex 15-2974

QC-082-MRS

March 4, 1988

Mr. S. R. Briggs

Hestinghouse Hanford Company

Post Office Box 1970

Richland, Hashington 99352

Dear Steve:

\section{PIIL IMSPECTION CAPABILITIES FOR GROUND-WATER MOMITORING HELL CONSTRUCTION}

PML currently provides geological support during ground-water monitoring well construction to oversee the construction, provide liquid and sediment sampling, record lithology, and provide expert geological guidance concerning construction, development and completion. Reclassification of the construction activities as Impact Level II by WHC has required the application of ANSI/ASME KiQA-1 quality assurance requirements. Specifically, in this instance; the application of Basic Requirement 10, Inspection, and Supplement 10S-1 will be specified in. the statement of work.

Because of their intimate knowledge and expertise in the area of monitoring svell construction, PNL proposes the use of PML site geologists to perform applicable inspections of Impact Level II well drilling/installation activities.

\section{Qualification}

PIL site geologists are already considered qualified to perform most of the inspections involved with mell construction. However, it is recognized they inay int be versed in typical inspection practices such as inspection forms, accept/reject practices and reporting methods. Although site geologists wil1 not be:certified in accordance with PML Administrative Procedure PAP-70-203, "Qualification and Certification of Inspection/Test and MOT Personnel," PHL's QC Section will provide docunented orientation to the site geologists of inspection practices/methods and provide oversight of the well monitoring/ ilspections by performing periodic surveillances. Training/qualification of jeologist will be documented per PAP-70-201, Indoctrination and Training, Parugraphs $4.2 .3,4.2 .4$, and 4.3.1. 
Mr. S. R. Briggs

March 4, 1988

Page 2

\section{Method/Documentation}

PHL Technical Procedure P-6, Revision 0 Procedure for Collection and Doculnentation of Drilling Data for RCRA Compliance/Hazardous Materials Monicoring, is currently applied by PNL site geologists to document well consiruction data. Attachment $A$ of the procedure, Hell Completion/Inspection Rogurt, has been modified to identify the inspection method used, acceptance criberia, and space for recording objective evidence of inspection results.

Records

Records will identify:

- item inspected

- date of inspection

- inspector

- type of observation

- results or acceptability

- references to information on action taken in connection with nonconformances

Ins!gection records will be maintained and will be supplied to XEH and HHC upon request.

Critical Areas For thich PHL Geologists Hill Provide Inspection

Verification that critical specifications are met will be provided for the following areas:

- drilling method

- Tubricants/drilling aids

- cleaning of well materials

- installation of permanent well screen

- installation of permanent casing

- drillers' log maintenance

- filter pack placement

- secondary seal placement

- placement of cement grout

- cement grout preparation

- material storage/factory seals

- well development

- well straightness

- installation of surface protection 
Mr. S. R. 8riggs

March 4, 1988

Page 3

The PML site geologist will not verify the following areas:

- receiving inspection of procured materials/material certifications

- drillers' licenses/qualifications

- records requi red by HAC 173-160-050

- welding of temporary casing to ASME or AHS codes

If the inspection process out]ined here meets your needs and expectations, please indicate your concurrence below and return a signed copy of this letter to me.

Sincerely,<smiles>CC1CC1CC1CC1</smiles>

R. M. Saith, Manager

Site Characterization and Assessment Section

RHS/ORD/r1
cc: R Clayton - WHC
DR Dahl
MD Freshley
WW Graham
PJ Mitchell
IH Horden

Concurrences:
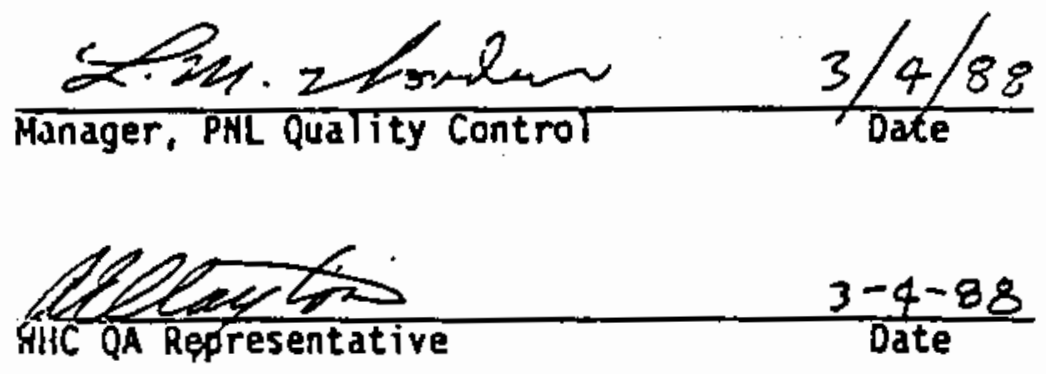

$\frac{\text { AR Brige }}{\text { WHC Technical/Representative }} \frac{3-4-38}{\text { Date }}$ 
WELL COMPLETION/INSPECTION REPORT

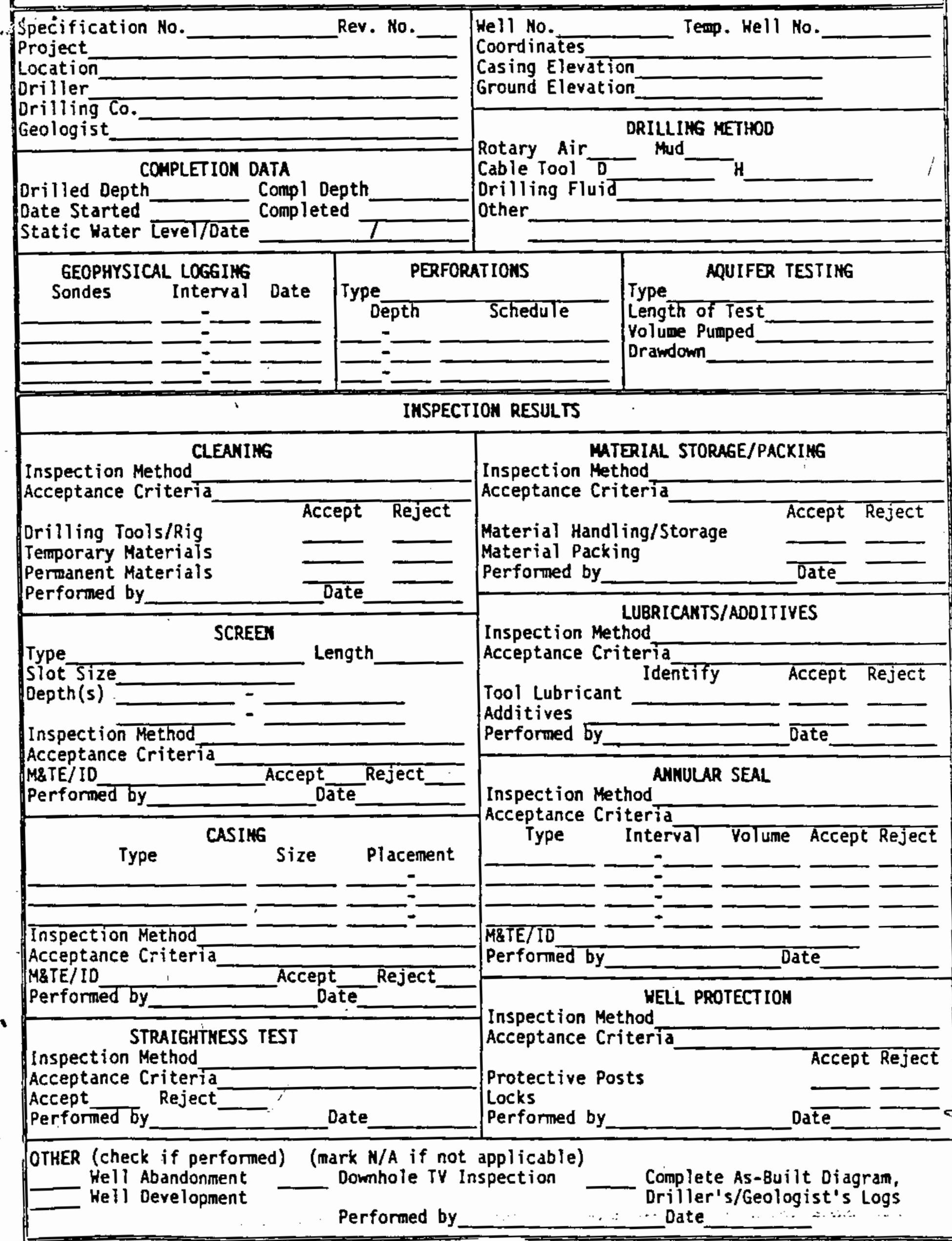


FECOMMENDED TRAINING FOR GEOLOGIST QUALIFICATIONS

$$
F \cdot A F-70-201
$$

1. TYFE OF TRAINING SFECIFIED

$$
\begin{aligned}
& \text { FARAGRAFH 4.2.1 FOFMAL TRAINING - EXHIEIT 1. FG } 1 \\
& \text { PAFIAGRAFH 4.2.2 OJT - EXHIEIT 1. FG } 4 \\
& \text { * FafiagrafH 4.2.5 ERIEF INGS - EXHIEIT } 2
\end{aligned}
$$

NO'TE: FEADING ASSIGINMENTS WOULD BE FOR THOSE ACTIUITIES FEQUIRING THE USE OF TECHNICAL FFIOCEDURES. (e.Q. Cement grout preparation. Secondary seal olacement. ETC ...)

2. ESTAELISH OF COMPETENCY

* PAFFgRafH 4.3.1 RESUME dF other formal document

- DATE

- EDUCATION

- WOFK EXPERIENCE

- LICENEE \& CERTIFICATIONS

- FELATED TFAINING * QUALIFICATION

* recommended tFaining and COMfetency verificatian

CC: DR Dahl

LM Worden

\section{so N-}


Date September 21, 1988

To Distribution

From

RW Bryce

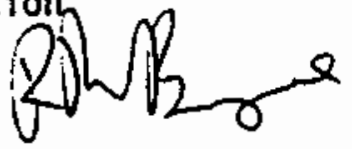

Subject Interim Change to Procedure $P-6$, Collection and Documentation of Drilling Data for RCRA Compliance/Hazardous Materials Monitoring

Although the new ground-water monitoring technical procedure manual, PNL-MA567 will soon be released replacing all existing ground-water monitoring technical procedures, recent developments require an interin change to procedure P-6, Collection' and Documentation of Drilling Data for RCRA Compliance/Hazardous Materials Monitoring, in order to reflect client requirements. Please follow the instructions on the attached Interim Change Notice (ICN) concerning changes to the procedure. These changes have been incorporated into the new procedure to be contained in PNL-MA-567 which replaces P-6. If you have any questions please contact either myself or Dave Dahl.

RWB/DRD/mp 
A. Doc. No. P-6_Rev. No. $\quad 0$ Doc. Title Data for RCRA Compliance/Hazardous Materials

Document's Author TL Liikala

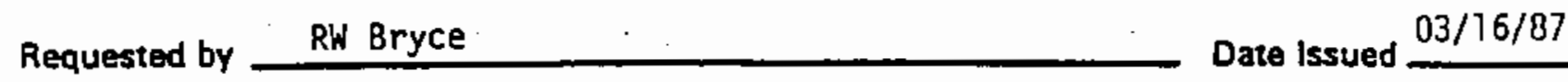

8. Type of Change and Approvals:

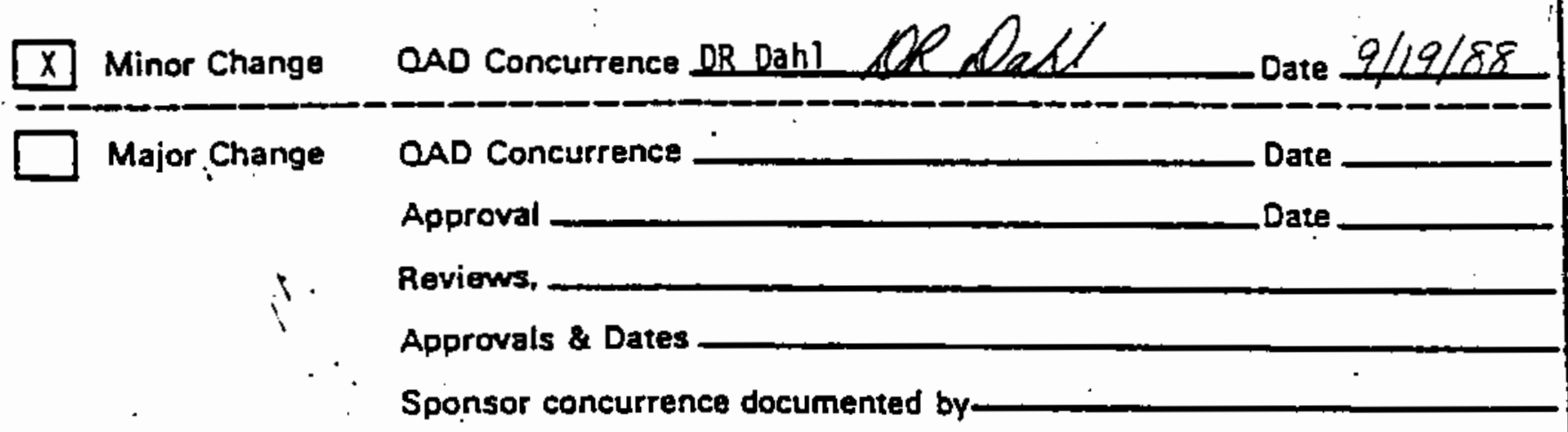

C. Reason for Change:

a) Through negotiations with WHC concerning the role and responsibilities of the PNL Well-Site-Geologist during well construction it was concluded that the PNL WellSite-Geologist does not perform Title III Inspection. The Well-site-Geologist provides an inspection capability verifying only aspects in his/her-area of expertise are being met. Therefore, references to Title III Inspection will be deleted.

b) In order to more accurately define the necessary well construction data that must be recorded and allow for documentation of Well-Site-Geologists' inspection results the Well Completion Report/Title III Inspection List (Attachment 1 of P-6) will be replaced by the attached Well Completion/Inspection Report.

D. Description of Change (old text versus requested test):

a) Replace Attachment 1 , Well Completion Report/Tjtle III Inspection List, page 1 of 1 of the referenced document with the attached Well Completion/Inspection Report.

b) Change all references to the "Well Completion Report/Title III Inspection List"

to. "Well Completion/Inspection Report."

- place this ICN at the fRont of the subject document"* 


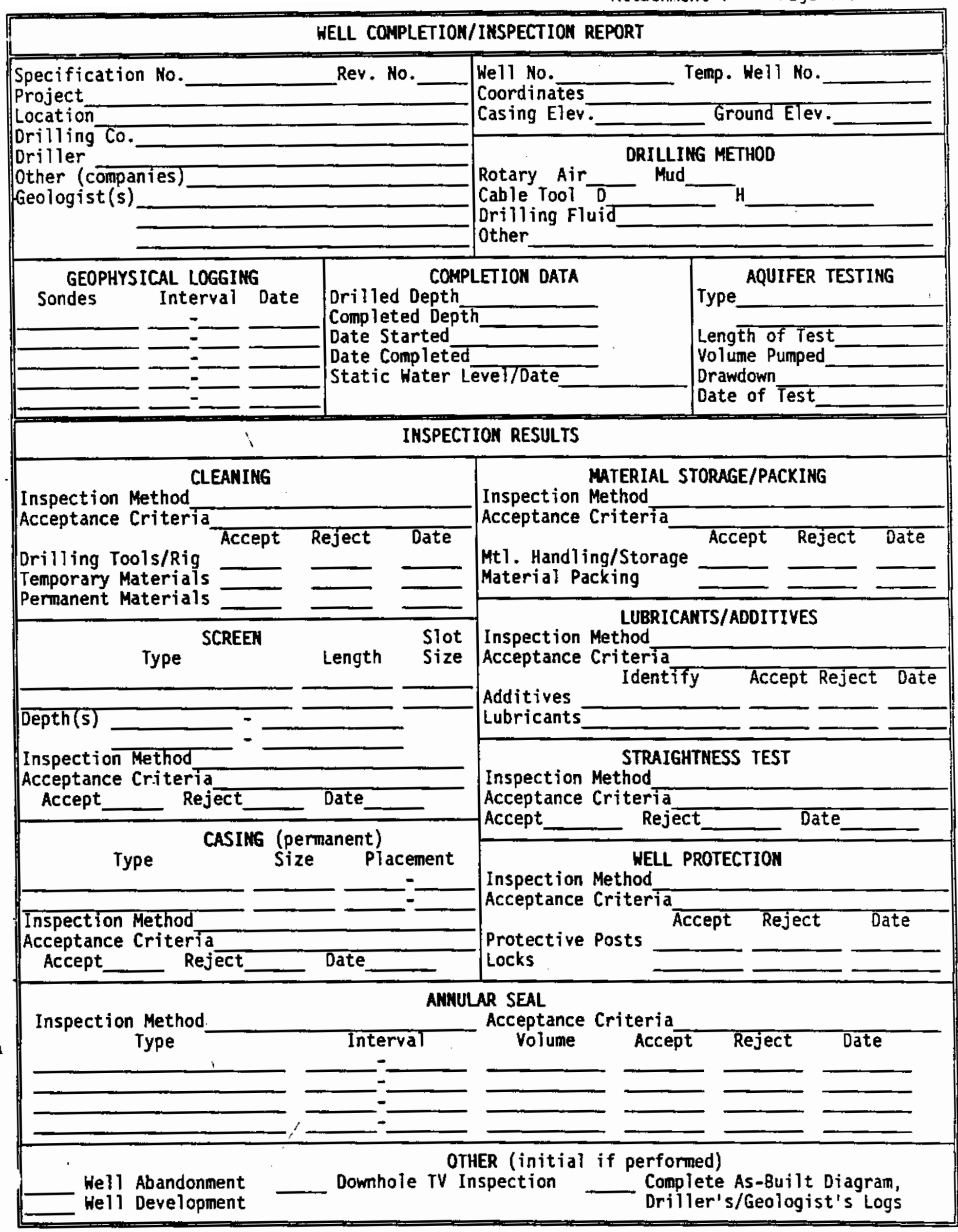

For all blanks mark N/A if not applicable. 



\section{DISTRIBUTION}

No. of

Copies

OFFSITE

2 DOE/Office of Scientific and Technical Information

\section{ONSITE}

2 DOE Richland Operations office

R. D. Izatt

M. L. Tiernan

6 Westinghouse Hanford Company

G. Carpenter

K. R. Fecht

R. L. Jackson

K. N. Jordan

R. G. McCain

L. C. Swanson
No. of

Copies

20 Pacific Northwest Laboratory

R. W. Bryce (2)

J. W. Falco

R. E. Gephart

S. M. Goodwin

J. M. Hales

P. C. Hays

R. E. Lundgren

P. J. Mitchell

R. L. Skaggs

R. M. Smith

J. A. Stottlemyre

S. S. Teel

R. G. Wildung

Publishing Coordination

Technical Report Files (5) 
. 